Marquette University

e-Publications@Marquette

Master's Theses (2009 -)

Dissertations, Theses, and Professional Projects

\title{
Computational Characterization of the Cellular Origins of Electroencephalography
}

Shane Hesprich

Marquette University

\section{Recommended Citation}

Hesprich, Shane, "Computational Characterization of the Cellular Origins of Electroencephalography" (2019). Master's Theses (2009 ). 533 .

https://epublications.marquette.edu/theses_open/533 


\section{COMPUTATIONAL CHARACTERIZATION OF CELLULAR CONTRIBUTIONS TO ELECTROENCEPHALOGRAPHY}

By

Shane Hesprich

A Thesis submitted to the Faculty of the Graduate School, Marquette University, in Partial Fulfilment of the Requirements for the Degree of Master of Science

Milwaukee, Wisconsin

May 2019 
ABSTRACT

\title{
COMPUTATIONAL CHARACTERIZATION OF CELLULAR CONTRIBUTIONS TO ELECTROENCEPHALOGRAPHY
}

\author{
Shane Hesprich
}

\section{Marquette University, 2018}

Electroencephalography (EEG) is a non-invasive technique used to measure brain activity. Despite its near ubiquitous presence in neuroscience, very little research has gone into connecting the electrical potentials it measures on the scalp to the underlying network activity which generates those signals. This results in most EEG analyses being more macroscopically focused (e.g. coherence and correlation analyses). Despite the many uses of macroscopically focuses analyses, limiting research to only these analyses neglects the insights which can be gained from studying network and microcircuit architecture. The ability to study these things through non-invasive techniques like EEG depends upon the ability to understand how the activity of individual neurons affect the electrical potentials recorded by EEG electrodes on the scalp. The research presented here is designed to take the first steps towards providing that link.

Current dipole moments generated by multiple multi-compartment, morphologically accurate, three dimensional neuron models were characterized into a single time series called a dipole response function (DRF). We found that when the soma of a neuron is directly stimulated to threshold, the resulting action potential caused an excess of current which backpropagated up the dendritic tree activating voltage gated ion channels along the way. This backpropigation created a dipole which had a magnitude an duration greater than the current dipoles created by neurons that were synaptically activated to near threshold.

Additionally, we presented a novel technique, where, through the combination of the DRFs with point source network activity via convolution, dipoles generated by populations of neurons can be simulated. We validated this technique at multiple spatial scales using data from both animal models and human subjects. Our results show that this technique can provide a reasonable representation of the extracellular fields and EEG signals generated in their 
physiological counterparts. Finally, analysis of a simulated evoked potential generated via the convolutional methodology proposed showed that $\sim 98 \%$ of the variability of simulated signal could be accounted for by the dipoles originating from DRFs of spiking pyramidal cells. 
TABLE OF CONTENTS

TABLE OF CONTENTS

LIST OF FIGURES $\quad$ iv

$\begin{array}{lll}\text { LIST OF TABLES } & \text { v }\end{array}$

\begin{tabular}{lll}
\hline & Introduction and Specific Aims & 1
\end{tabular}

$1.1 \quad$ Introduction to Electroencephalography . . . . . . . . . . . . . 1

$1.2 \quad$ Specific Aims . . . . . . . . . . . . . . . 2

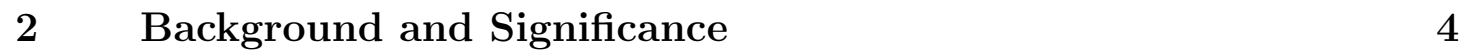

$2.1 \quad$ Extracellular Currents . . . . . . . . . . . . . . 5

2.1 .1 Ionic Currents . . . . . . . . . . . . . . . 5

$2.1 .2 \quad$ Synaptic Currents . . . . . . . . . . . . . . . . . . 11

$2.2 \quad$ Cellular Morphology and Electric Fields . . . . . . . . . . 15

$2.3 \quad$ Current Dipole Moments and the Forward Projection . . . . . 19

$2.4 \quad$ Network Dynamics . . . . . . . . . . . . . . . . 23

2.4 .1 Single Compartment Neuron Models . . . . . . . . . 23

2.4 .2 Networks of Point Source Neurons . . . . . . . . . . . . 24

\begin{tabular}{lll}
\hline & Characterizing Cellular Dipoles & 26
\end{tabular}

$3.1 \quad$ Cell Models . . . . . . . . . . . . . . . . . . . . . . . . 26

$3.1 .1 \quad$ Calculation of Current Dipole Moment . . . . . . . . . 29

$3.2 \quad$ Dipole Response Functions . . . . . . . . . . . . . . . . . . . . 32

3.2.1 Comparing spiking and synaptic contributions . . . . . 41

3.3 $\quad$ Discussion . . . . . . . . . . . . . . . . . . . . . . . . 43

$4 \quad$ Simulation of EEG Using DRFs 46 
$4.1 \quad$ Calculation of the Population Dipole . . . . . . . . . . . . . 46

4.1 .1 Thalamocortical Network Model . . . . . . . . . . . . 46

$4.1 .2 \quad$ Population Dipole . . . . . . . . . . . . . . . . . . . . . . 49

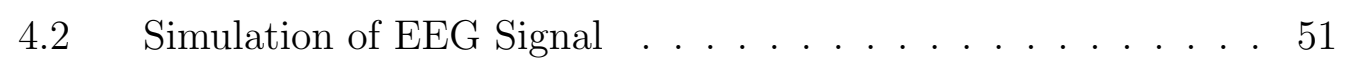

4.2 .1 Patient Data . . . . . . . . . . . . . . 51

$4.2 .2 \quad$ Forward Projection of Simulated Activity . . . . . . . . 52

4.3 Comparison of Simulations to Physiologic Data . . . . . . . . 53

4.3 .1 Network Activity and the Population Dipole . . . . . . 53

4.3 .2 EEG Activity . . . . . . . . . . . . . . . . . 55

$4.4 \quad$ Discussion $\ldots \ldots \ldots \ldots \ldots$

$\begin{array}{lll}5 & \text { Evoked Potentials } & 59\end{array}$

$5.1 \quad$ Thalmocortical Evoked Response . . . . . . . . . . . . . . 59

5.1 .1 Population Dipole . . . . . . . . . . . . . . . . . 60

5.1 .2 Patient Data . . . . . . . . . . . . . 61

5.1 .3 Source Localization . . . . . . . . . . . . . . 61

$5.2 \quad$ Analysis $\ldots \ldots \ldots \ldots$. . . . . . . . . . . . . . 62

$5.2 .1 \quad$ Bootstrap Analysis . . . . . . . . . . . . . 62

5.2 .2 Correlation of Decomposed Simulated Signal . . . . . . 62

$5.3 \quad$ Evoked Potential . . . . . . . . . . . . . . . . . . . . . 63

5.4 Discussion . . . . . . . . . . . . . . . 66

\begin{tabular}{lll}
\hline 6 & Discussion & 68
\end{tabular}

\begin{tabular}{lll}
\hline 7 & Conclusion & 71
\end{tabular}

$7.1 \quad$ Future Directions . . . . . . . . . . . . . . . . . . . 72

\begin{tabular}{|ll}
\hline A & Appendix: Cellular Currents \\
\hline
\end{tabular}

A.1 NEURON Models . . . . . . . . . . . . . . . . . . . . . . . 74 
A.2 Point Source Models . . . . . . . . . . . . . . . . . 78

A.2.1 Pyramidal Cell . . . . . . . . . . . . . . 84

A.2.2 Interneuron . . . . . . . . . . . . . . 86

A.2.3 Reticular Neuron . . . . . . . . . . . . . . . 87

A.2.4 Thalmocortical Cell . . . . . . . . . . . . . . . 87

\begin{tabular}{lll}
\hline B & Synaptic Models & 88
\end{tabular}

B.1 Point Source Models . . . . . . . . . . . . . . . . . . . . 88

B.1.1 GABA Type A . . . . . . . . . . . . . 88

B.1.2 GABA Type B . . . . . . . . . . . . . . . . . 89

B.1.3 AMPA . . . . . . . . . . . . . . . . . 990

B.1.4 AMPA Type D2 . . . . . . . . . . . . . . . . . . 91

B.1.5 NMDA Type D1 . . . . . . . . . . . . . 93

B.1.6 GABA Type A D2 . . . . . . . . . . . . . . . . 94

B.1.7 Externally stimulated AMPA. . . . . . . . . . . . . 96

\begin{tabular}{lr}
\hline Bibliography & 98
\end{tabular} 


\section{LIST OF FIGURES}

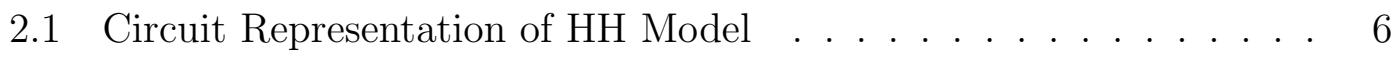

$2.2 \quad$ Illustration of Synapse $\ldots \ldots \ldots \ldots$

2.3 Neuron Morphologies . . . . . . . . . . . . . . . . . . . . . . . . . . 16

2.4 Cable Theory Diagram $\ldots \ldots \ldots \ldots$

2.5 Neocortical Pyramidal cell modelled as a current dipole . . . . . . . 20

2.6 Cortex, skull, and scalp diagram . . . . . . . . . . . . . . . 21

2.7 Boundary Element Model . . . . . . . . . . . . . . . . . . 22

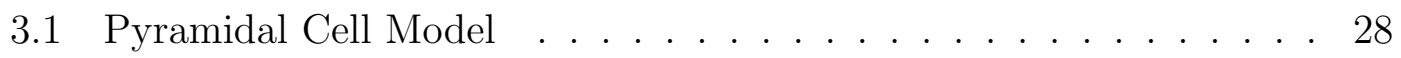

3.2 Dipole Response Functions . . . . . . . . . . . . . . . . . . . . . . . 33

3.3 Time-lapse of Regularly Spiking Pyramidal Cell . . . . . . . . . . . 35

3.4 Time-lapse of Spiny Stellate Cell . . . . . . . . . . . . . . . . . 36

3.5 Time-lapse of Chattering Pyramidal Cell . . . . . . . . . . . . . . . 37

3.6 Compartmental Breakdown of State Variables . . . . . . . . . . . . 39

3.7 Synaptic Dipoles . . . . . . . . . . . . . . . . . . . . . . . . 42

4.1 Thalamocortical Network Diagram . . . . . . . . . . . . . . . . . . 48

4.2 Pyramidal Cell Membrane Voltage Traces . . . . . . . . . . . . . . 54

4.3 Population Dipole Vs Depth EEG . . . . . . . . . . . . . . . . . . . 55

4.4 Comparison of SWS Time Series . . . . . . . . . . . . . . 56

4.5 Comparison of SWS Periodograms . . . . . . . . . . . . . . . 56

5.1 Comparison of Evoked Potentials . . . . . . . . . . . . . . . . . 64

5.2 Components of Evoked Response . . . . . . . . . . . . . . . 65 


\section{LIST OF TABLES}

3.1 Synaptic Conductances . . . . . . . . . . . . . . . . . . . 41

4.1 Network Topology $\ldots \ldots \ldots$. . . . . . . . . . . . . . . . . . . . 47 


\section{Introduction and Specific Aims}

\subsection{Introduction to Electroencephalography}

Electroencephalography (EEG) is a commonly used technique to measure electrical activity of the brain. It is used clinically to diagnose conditions such as epilepsy, encephalitis, encephalopathy, memory problems, strokes, sleep disorders and dementia. In research, EEG has permeated almost every area of neuroscience, from mechanistic research into motor control and sensory perception, to gross brain functions like cognition or Alzheimer's Disease.

Though the first human EEG was recorded in 1924 by German Psychiatrist Hans Berger (Haas, 2003), modern understanding of the origins of EEG signals did not begin until 1947 when Rafeal Lorente de No published his famous paper 'Analysis of Distributions of the Action Currents of Nerve in Volume Conductors' (Lorente de No, 1947). The paper provided an in-depth discussion and mathematical analysis of the fields generated by individual neurons as well as layers of neurons. The subsequent characterization of ionic channels by Drs. Alan Hodgkin and Andrew Huxley (Hodgkin and Huxley, 1952), formed the foundation for understanding how neurons create the extracellular currents that contribute to the electric fields characterized by Lorente de No. In spite of research efforts to characterize the exact origins of EEG signals Contreas and Steriade, 1995; Murakami et al., 2002), there has yet to be a definitive exploration of how the extracellular currents of individual neurons contribute to the gross electrical potentials detected on the scalp. A widely accepted view is that EEG signals result from the post synaptic potentials in synchronously firing pyramidal cells on the cortex of the brain (Lopes da Silva, 2010; Olejniczak, 2006). This interpretation of the physiologic origins of EEG is justified, in part, 
by the argument that the short duration of action potentials (1-2 ms) does not lend itself the the summative effect needed to produce a recordable signal at the scalp. Post synaptic potentials persist for a much longer period of time ( 20-40 ms) (Lopes da Silva, 2010; Olejniczak, 2006). However, these commonly held assumptions are based on the voltage changes recorded from the soma of neuron. This can be misleading since the potentials recorded on the scalp come form the extracellular currents generated by the cells, which may not relate directly to the voltage changes in soma. The extracellular currents which are generated by neuronal activity, can be characterized as a current dipole moment. These dipole moments can be significantly different than the voltage traces themselves (Murakami and Okada, 2006).

The objective of the research reported here is to investigate the assumption that EEG signals originate from the post synaptic potentials incident in synchronously firing pyramidal cells on the cortex of the brain. We will use computational techniques to quantify the contributions of the spiking and post synaptic activity of both pyramidal cells and inhibitory interneurons to determine how they influence the current dipoles generated by populations of neurons in the brain.

\subsection{Specific Aims}

In order to conduct a computational investigation like the one suggested above we must determine how populations of neurons generate current dipole moments, commonly considered the source of EEG signals. To determine the contributions of cell types and cellular events to the current dipoles generated in the brain, the research addressed the following aims. 


\section{Aim 1: Characterize the extracellular currents generated by multiple neuron types under a variety of states using a morphologically constrained dipole model. In order to investigate the} neural generators of EEG signals we must characterize how neurons generate dipoles. Using the NEURON computational framework (Carnevale and Hines, 2006), the detailed electrophysiological properties of three dimensional neurons can be simulated (Mainen and Sejnowski, 1996). Using techniques developed by Shingo Murakami (Murakami and Okada, 2006) simulations of three dimensional neuron morphology and activity can be used to calculate the current dipole generated by the neuron. This technique allows for all the electrophysiological and morphological complexity to be reduced to a single time series, which we refer to as a dipole response function (DRF). Dipole response functions will be generated for multiple neuron types and multiple cellular eventsin order to determine how each event on different neurons contribute to the total dipole.

\section{Aim 2: Develop a dipole model of extracellular currents} generated by populations of dynamically interacting neurons that accounts for different neuron types and cellular events. While using morphologically accurate neurons to simulate the electrophysiology of a single neuron is feasible, creating a network of such neurons is computationally intensive. In order to ease the computational load, point source neurons can be used to create a realistic network model. The network activity generated by the point source network can then be combined with the DRFs from aim 1 to generate a population level dipole. To validate the approach we used an established thalamocrotical model developed by Bazenhov et al. (2002). This model was simulates a patch of somatosensory patch cortex during active and slow wave sleep states. This model was chosen for two reasons. First, it is relatively simple with only four neural populations and a simple connection 
topology. Second, the network was built using intercellular recordings from a cat (Contreas and Steriade, 1995). Data from Contreas and Steriade (1995) can be used to validate that the dipole activity generated by this population of neurons is truly reflective of physiologically plausible underlying network activity.

\section{Aim 3: Use a dynamic transient network, in the form of an} evoked response, to determine how the different cell types and cellular events contribute to the population dipole. In this aim we use the same thalamocortical network as in aim 2, but modify it to generate an evoked potential. After verifying the accuracy of the simulated signal against a recorded evoked potential, the simulated signal can be broken down into its constitutive signals. Finally, we can determine the contributions of cell type and cellular events to the population dipole by comparing each constitutive signal to the summed total. Additionally, this shows that the technique has predictive capabilities (i.e. it was built to mimic SWS activity, but with minimal modification and no a priori knowledge of network dynamics can mimic other states).

\section{Background and Significance}

Electroencephalography is a passive physiological recording of electrical potentials on the scalp. Electrodes are placed on the scalp to record potential differences between locations on the scalp. These measured potentials are the result of electrical currents generated in the brain and propagate to the scalp. To interpret the brain activity associated with the potentials recorded by EEG electrodes, it is important to first understand how these currents are generated.

This section examines the state of knowledge regarding how individual 
neurons generate current dipoles, how those individual neurons organize into populations which create larger dipoles, and how dipole currents propagate through the brain to be detected by the electrodes on the scalp. Each subsection is geared towards a specific element of this process, to develop a cohesive physiological and mathematical understanding of EEG signals from neuron to scalp.

\subsection{Extracellular Currents}

Electric fields in the brain originate primarily from currents generated by electrically active cells known as neurons. Neurons consist of three major components; the soma, the dendrites, and the axon. The cell body of a neuron, also known as the soma, which contains the cell's nucleus, but also plays a crucial role in integrating and processing signals from other neurons. The dendrites are small branched protrusions from the cell body whose primary role is to receive signals from other neurons via synapses. The axon is a single projection from the soma used to send electrical signals to other neurons.

Neurons generate currents by either the transportation or diffusion of charged ions across the cell membrane. It is the current flowing outside of the cell (extracellular currents) which results in a detectable electric current. There are two main sources of extracellular currents; ionic currents (sometimes referred to as intrinsic currents), and synaptic currents.

\subsubsection{Ionic Currents}

One of the first mathematical characterizations of the transport process came from Drs. Alan Hodgkin and Andrew Huxley's analysis of the axon of a 


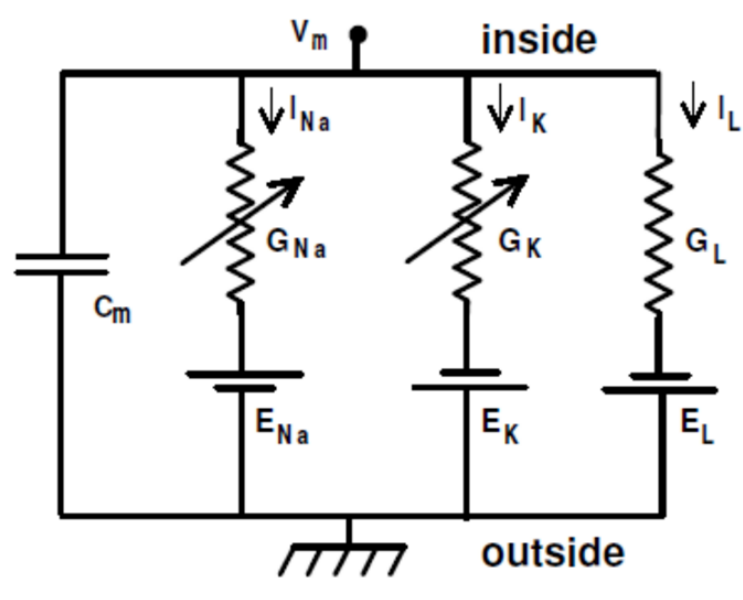

Figure 2.1: Circuit representation for the Hodgkin Huxley model of ion transport across a cell membrane. Electromotive forces for each ion species is modelled as the voltage sources. Conductances are modelled as resistor elements, and the lipid bilayer is modelled as the membrane capacitance. Two items of importance to note are the arrows through $G_{N a}$ and $G_{K}$ denoting they have variable conductances. Also note that $E_{N a}$ is facing in the opposite directions as $E_{K}$ and $E_{L}$. This is because the Nernst potential (voltage source) of sodium is positive while the Nernst potentials for potassium and leakage currents are negative

giant squid (Hodgkin and Huxley, 1952; Lopes da Silva, 2010; Olejniczak, 2006). By using a voltage patch clamp and varying the extracellular concentrations of potassium and sodium, they were able to mathematically characterize several important elecrophysiological attributes of the cell including how neurons control the flow of ions across the cell membrane. These findings were organized into a working mathematical model of a cell known as the Hodgkin-Huxley (HH) model, which forms the foundation for understanding the electrophysiology of excitable membranes. Through the HH model, Hodgkin and Huxley predicted the existence of several protein structures that had not yet been discovered, including their physical attributes. Most notably the HH model predicted that sodium channels were dual gated, while potassium channels were controlled by a single gate. 
The model breaks down the electrical characteristics of the neuron's cell membrane into several constituent components which can be modelled as electrical elements (see figure 2.1). The cellular membrane can be modelled as a capacitor with the extracellular and intercellular surfaces of the membrane acting as the faces of the capacitor and the lipid bilayer acting as the dialectic insulator. Mathematically, the current flow across the membrane can be modelled as follows:

$$
I_{c}=C_{m} \frac{d V_{m}}{d t}
$$

Where $I_{c}$ is the capacitive current flow, $C_{m}$ is the membrane capacitance and $\frac{d V_{m}}{d t}$ is the derivative of the voltage across the membrane with respect to time.

The electromotive force (or Nernst potential) for each ion species is represented schematically as a constant voltage source. The Nernst potentials of sodium, $E_{N a}$, and potassium, $E_{K}$, are explicitly represented. Other ion species all lumped into a leakage potential, $E_{L}$.

A Nernst potential develops when an electrolytic solution is placed in a bath separated by a semipermeable membrane (i.e. a membrane which is permeable only to specific ions). This results in two opposing forces. The first force is osmotic pressure, which is driven by the concentration gradient. If the two sides of the bath have unequal concentrations of ions, the ions will naturally diffuse across the membrane in order to achieve isotonic equilibrium. The second driving force is an electromotive force. Ions, by definition, are charged. Thus, when separated by a semipermeable membrane, if they develop a concentration gradient, they also develop a potential difference. The electrical potential developed across the semipermeable membrane at which these two forces reach equilibrium for a given ion species is called a Nernst potential, $E$. 
Mathematically it can be represented as:

$$
E=\frac{R T}{z F} \ln \left(\frac{[S]_{\text {out }}}{[S]_{\text {in }}}\right)
$$

where $R$ is the ideal gas constant, $T$ is the temperature in kelvins, $F$ is Faraday's constant in Coulombs per mole and $z$ is the sign and elementary charge of the ion. Finally, $[S]_{\text {out }}$ and $[S]_{\text {in }}$ are the outer and inner concentrations of ion species $S$ respectively.

Despite these driving forces, ions cannot pass directly through the lipid bilayer which separates the extracellular medium from the intercellular medium. They must pass through special transmembrane proteins known as ion channels, which provide a path for ions to pass from one side of the membrane to the other. Passive channels constantly remain open and allow for the free flow of ions. The concentrations of these channels on the surface as well as the ease with which ions can flow through them are modelled in the HH model as the leakage conductance, or $G_{L}$. Active channels, on the other hand, open and close dynamically based on a variety of circumstances. The most common of these respond to the voltage difference across the cell membrane, and are referred to as voltage-gated ion channels. The giant squid axon represented by the HH model had two voltage-gated channels; a sodium channel and a potassium channel. The permittivity of each ion species is mathematically represented as a conductance and is described by the following equations:

$$
\begin{aligned}
G_{N a} & =\bar{g}_{N a} m^{3} h \\
G_{K} & =\bar{g}_{K} n^{4}
\end{aligned}
$$

where, $G_{N a}$ and $G_{K}$ are the conductances of sodium and potassium channels 
respectively. The variables $\bar{g}_{N a}$ and $\bar{g}_{K}$ denote the maximum conductance when all channels are open. The variables $m, n$, and $h$ are voltage dependant gating variables, whose rates of change are characterized by first order differential equations defined with respect to the membrane voltage $\left(V_{m}\right)$.

$$
\begin{aligned}
\frac{d n}{d t} & =\alpha_{n}\left(V_{m}\right)(1-n)-\beta_{n}\left(V_{m}\right) n \\
\frac{d m}{d t} & =\alpha_{m}\left(V_{m}\right)(1-m)-\beta_{m}\left(V_{m}\right) m \\
\frac{d h}{d t} & =\alpha_{h}\left(V_{m}\right)(1-h)-\beta_{h}\left(V_{m}\right) h
\end{aligned}
$$

where $\alpha$ and $\beta$ are voltage dependant rate constants given by

$$
\begin{aligned}
\alpha_{n}\left(V_{m}\right) & =\frac{0.01\left(V_{m}+10\right)}{\exp \left(\frac{V_{m}+10}{10}\right)-1} \\
\beta_{n}(V m) & =0.125 \exp \left(\frac{V_{m}}{80}\right) \\
\alpha_{m}\left(V_{m}\right) & =\frac{0.1\left(V_{m}+25\right)}{\exp \left(\frac{V_{m}+25}{10}\right)-1} \\
\beta_{m}(V m) & =4 \exp \left(\frac{V_{m}}{18}\right) \\
\alpha_{h}\left(V_{m}\right) & =0.07 \exp \left(\frac{V_{m}}{20}\right) \\
\beta_{h}(V m) & =\frac{1}{\exp \left(\frac{V_{m}+30}{10}\right)+1}
\end{aligned}
$$

for the giant squid axon. From Ohm's Law the flow of ions across the membrane can be modelled as a current.

$$
\begin{aligned}
I_{N a} & =\bar{g}_{N a} m^{3} h\left(V_{m}-E_{N a}\right) \\
I_{K} & =\bar{g}_{K} n^{4}\left(V_{m}-E_{k}\right)
\end{aligned}
$$

Combining equations 2.1, 2.10, and 2.11 to solve for the net current across the 
cell membrane, the change in membrane voltage is given by

$$
\frac{d V_{m}}{d t}=\frac{1}{C_{m}}\left(\bar{g}_{K} n^{4}\left(V_{m}-E_{K}\right)+\bar{g}_{N a} m^{3} h\left(V_{m}-E_{N a}\right)+g_{l}\left(V_{m}-E_{L}\right)-I\right)
$$

where $I$ is either an externally applied current or a synaptic current.

This model provides a quantitative description of how neurons control the flow of ions across the cell membrane to generate an action potential. It is these extracellular currents which form the basis of the electric fields which can be detected by EEG electrodes. The base HH model only describes voltage-gated sodium and potassium currents, but different cells types can have other ionic currents that contribute to the membrane voltage. Most can be characterized in the HH framework by a maximum conductance, $\bar{g}$, scaled by one or more gating variables, whose rate of change is governed by a first order differential equation, which is in turn dependent on two rate constants $\alpha$ and $\beta$. These rate constants can be dependant on a number of factors such as voltage or concentration of specific chemical species. Regardless of the number of different ionic currents

that contribute to a cell's membrane voltage, the sum of these currents define the total extracellular current which contribute to the current dipoles generated by the neuron.

\section{The Action Potential}

Action potentials are the means by which neurons communicate. They are generated by positive feedback of the sodium current. An influx of sodium ions causes the cell membrane to depolarize. The depolarizing of the cell membrane opens the voltage gated sodium channels allowing more sodium to flow into the cell. As long as the influx of sodium ions results in a current which is less than the leakage current, the cell will return to its resting potential. However, if the 
cell depolarizes to a level where the sodium current is greater than the leakage current, then the sodium current causes a positive feedback loop. The voltage at which this occurs is called the threshold potential. Once the threshold potential is reached the positive feedback of sodium current continues until the membrane potential gets high enough that the secondary sodium gate closes, halting the influx of sodium ions. At around the same time the secondary sodium gates close the potassium gates open. This causes potassium ions to flow out of the cell, repolarizing the cell membrane, and once it reaches resting levels the gates reset. The cycle of positive feedback and return to resting potential results in an action potential and is sometimes referred to as a spike, since a voltage trace of the membrane potential resembles a spike. The importance of the action potential is that once the soma of the cell reaches the threshold potential from dendritic inputs, the cell not only spikes, but the sodium currents from the positive feedback diffuse into the axon of the neuron. This causes the portion of the axon closest to the soma to depolarize beyond threshold potential and spike as well. Sodium currents from this spike cause nearby portions of the axon to spike such that the spike travels down the length of the axon where it terminates at a synapse on the dendrites of one or more other neurons.

\subsubsection{Synaptic Currents}

The primary method by which neurons in the cortex communicate with each other is through synapses. A synapse consists of three main components. The presynaptic terminal, the synaptic cleft, and the postsynaptic dendrite. An illustration of a synapse is shown in figure 2.2. When an action potential reaches the terminal point of an axon, or presynaptic terminal, special voltage-gated calcium channels allow for the flow of calcium ions into the cell. The influx of 


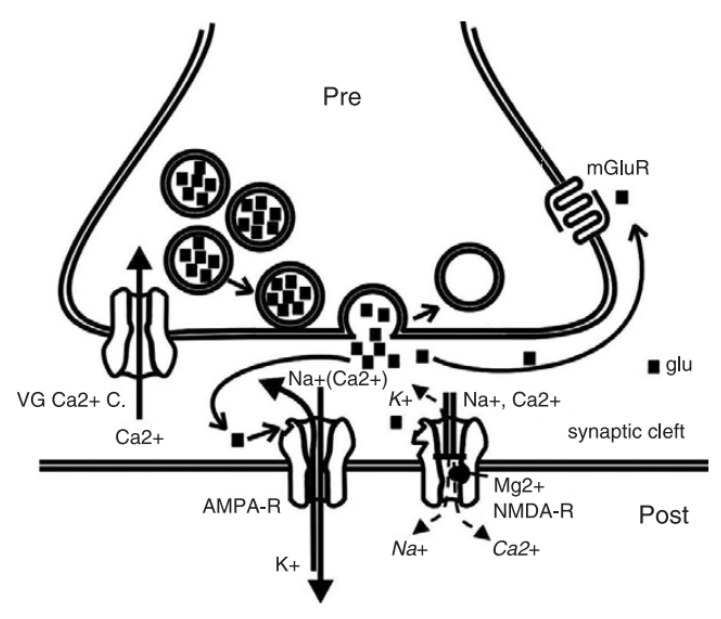

Figure 2.2: Schematic drawing of a synapse with presynaptic vesicles, voltage gated calcium channels, and postsynaptic receptors. Following membrane depolarization due to an action potential, voltage gated calcium channels activate and allow calcium into the cell. Calcium then binds to proteins inside the synapse which facilitate the release of the neurotransmitter glutamate (glu) into the synaptic cleft, which activated AMPA and NMDA ligand gated ion channel receptors on the post synaptic dendrite. Figure reproduced from (De Schutter, 2010).

calcium causes vesicles containing neurotransmitters to fuse with the cell membrane. This process releases neurotransmitters out of the cell into the synaptic cleft. The neurotransmitters then diffuse across the cleft and bind with ligand-gated ion channels on the postsynaptic neuron's dendrites. The binding of the neurotransmitters to the ligand-gated ion channels (sometimes called a receptor protein or neuroreceptor) allows for the flow of charged ions to pass into or out of the postsynaptic cell. The depolarization or hyperpolarization of the post synaptic cell in response to the flow of ions is referred to as a post synaptic potential. Each type of ligand-gated channel is permeable to a different ion species resulting in different changes in the membrane voltage of the postsynaptic neuron. An excitatory synapse is a synapse whose ligand-gated ion channel have a reversal potential above the threshold potential. AMPA receptors have an excitatory effect due to their equal permeability to sodium and 
potassium, which results in a reversal potential around $0 \mathrm{mV}$. An inhibitory synapse has channels with a reversal potentials below threshold, and often times below the membrane's resting potential. GABAA receptors have an inhibitory effect due to their selective permeability to chloride which results in a reversal potential of around $-70 \mathrm{mV}$.

Synapses can be modelled in one of two ways. The first is to approximate the synapse as a synaptic current. The synapse is reduced to a simple post synaptic current which is modelled as a decaying exponential (Weber et al., 2003).

$$
I(t)=\left(t-t_{0}\right)^{n} \exp \left(-\frac{t-t_{0}}{\tau^{P S C}}\right)
$$

Where $I(t)$ is the post synaptic current, $\tau^{P S C}$ is the time constant related to the decay of the post synaptic current, $n$ is an integer relating to the order of the decaying exponential, and $t_{0}$ is the time of the presynaptic spike.

A more physiologically accurate way to model a synapse is to use a conductance based model (De Schutter, 2010). This approach does not assume a decaying exponential current, but a decaying exponential conductance. Mathematically, this can be represented by

$$
I(t)=g(t)_{s y n}\left(V_{m}-E_{\text {syn }}\right)
$$

where $g(t)_{\text {syn }}$ is the time varying synaptic conductance and $E_{\text {syn }}$ is the synaptic reversal potential. The function $g(t)_{s y n}$ can take several different forms depending on the desired complexity of the model. The most basic model is a first order exponential decay of the conductance.

$$
g(t)_{s y n}=\bar{g}_{s y n} \exp ^{-\left(t-t_{0}\right) / \tau}
$$


where $\bar{g}_{\text {syn }}$ is the maximum conductance of the synapse and $\tau$ is the exponential time constant. The problem with a first order approximation is that the rise time is instantaneous. While this is a good approximation for certain fast acting receptors like an AMPA receptor, it may not accurately characterize other types of receptors. For a more generalizable model, an alpha function can be used,

$$
g(t)_{s y n}=\bar{g}_{\text {syn }} \frac{t}{\tau} \exp ^{1-\left(t-t_{0}\right) / \tau}
$$

The advantage of the alpha function is that it has a finite rise time. The drawback is that the rise time and decay time are coupled. In order to decouple rise and decay times a difference of exponentials can be used,

$$
g(t)_{s y n}=\bar{g}_{s y n} * f *\left(\exp ^{-\left(t-t_{0}\right) / \tau_{\text {decay }}}-\exp ^{-\left(t-t_{0}\right) / \tau_{\text {rise }}}\right)
$$

where $\tau_{\text {decay }}$ and $\tau_{\text {rise }}$ are the decay and rise time constants respectively, and $f$ is a normalization factor which ensures that the peak amplitude of $g(t)_{\text {syn }}$ is $\bar{g}_{\text {syn }}$ at the peak rise time. In this case

$$
\tau_{\text {peak }}=\frac{\tau_{\text {deacy }} \tau_{\text {rise }}}{\tau_{\text {decay }}-\tau_{\text {rise }}} \ln \left(\frac{\tau_{\text {decay }}}{\tau_{\text {rise }}}\right)
$$

and

$$
f=\frac{1}{-e x p^{-t_{\text {peak }} / \tau_{\text {rise }}}+e x p^{-t_{\text {peak }} / \tau_{\text {deacy }}}}
$$

Additional models of the synaptic conductance include, G-protein mediated receptors (De Schutter, 2010), and receptors that model short-term depression or excitation (Bazenhov et al., 2002). However, in general, the more complex the model, the more computational time it takes to simulate. Thus a trade is typically be made between physiological realism and reasonable computational times within the context of the scope, scale, and objectives of a study. 


\subsection{Cellular Morphology and Electric Fields}

In addition to the ionic currents generated through a patch of neuronal membrane or a single synapse, electric field potentials are also impacted by the physical shape of neurons, also referred to as cellular morphology. Each individual cell type has its own morphology. Some neurons, like spiny stellate cells contain small tufts of dendrites which resemble a tumble weed. Other neurons, like the layer IV pyramidal cell, have basil dendrites that extend down towards the deeper layers of the brain, and apical dendrites which extend up towards layer I of the cortex. Purkinje cells have a dendritic structure which spreads out in a like fan coral. Figure 2.3 shows a diagram of the different dendritic morphologies. The electric fields generated by individual neuron types are as varied as the morphologies of the neurons themselves.

Many qualitative assessments of how morphology relates to the generation electric fields have been done. Most notably in 1947 by Lorente de No (Lorente de No, 1947), and again in 1974 by Llinas (Llinas and Nicholson, 1974). Llinas specifically states that "neurons having dendrites which ramify radially from the soma ... tend to generate currents which flow radially in all directions and thus would tend to cancel." Cells such as these are often referred to as having "closed field" morphologies or as "closed field" neurons (Nunez and Srinivasan, 2006; Lopes da Silva, 2010; Llinas and Nicholson, 1974). Pyramidal cells and Purkinje cells, on the other hand have dendrites that are parallel to

each other, which leads to additive parallel currents (Llinas and Nicholson, 1974). These cells are often referred to as having an "open field" morphology or as "open field" neurons (Nunez and Srinivasan, 2006; Lopes da Silva, 2010; Llinas and Nicholson, 1974). It is noteworthy that many open field neurons have electric fields which tend to originate from the soma and extend out towards the 


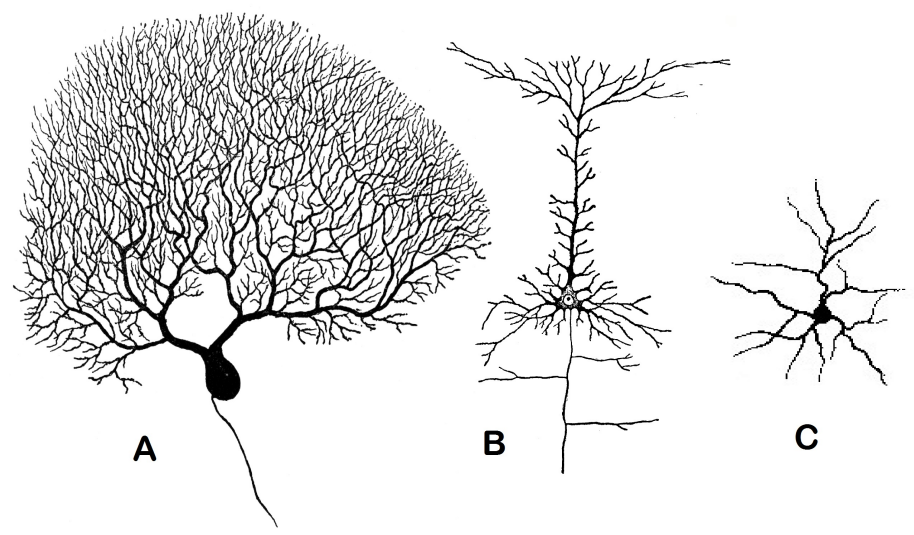

Figure 2.3: Morphologies of different neuron types. A) Purkinje Cell B) Pyramidal cell C) Stellate Cell. Figure modified from (Johns, 2014).

dendrites. This type of field can be modelled as a current dipole, with a current source located at the soma and a current sink located somewhere in the apical dendrites (Lopes da Silva, 2010). The importance of this dipole representation of a neuron will be discussed in the next section.

Quantitative characterization of the electric fields generated by neurons require a mathematical description of the propagation of electrical currents in both space and time. This was first done in the late 1940s and early 1950s in the form of Cable Theory. This theory gets its origins from the transatlantic telegraph. In 1854 Lord Kelvin published a mathematical model of the proposed transatlantic telegraph cable where he broke the cable down into discrete elements (Rall, 1959). Each element was represented as an equivalent electrical circuit, where currents from telegraph signals travelled from compartment to compartment down the length of the wire, while a certain amount of current leaked from the insulated housing (William, 1854). The concept was adapted to neurons, first by Alan Hodgkin (Hodgkin and Rushton, 1946), and later refined by Rafael Lorente de No (Lorente de No, 1947). Excitatory synapses cause 


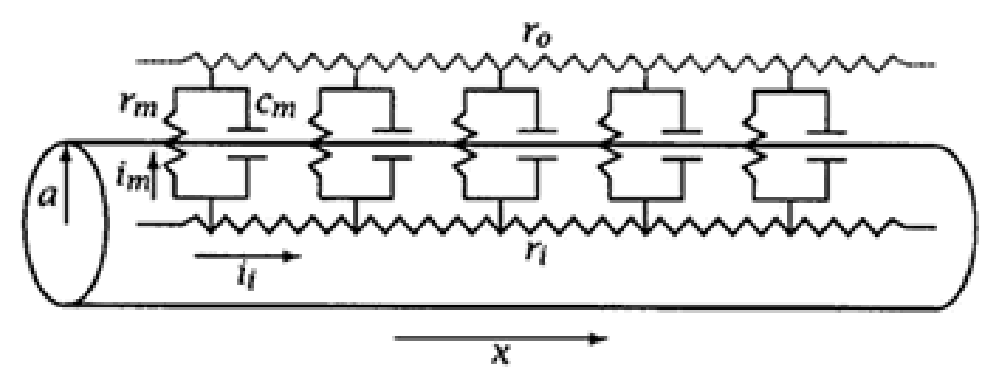

Figure 2.4: Cable Theory diagram showing the dendritic membrane modelled as simple RC circuits connected in parallel by internal and external longitudinal resistances. Figure reproduced from (Jaeger, 2005).

current to flow into the cell, where the current flows down the length of a conductive core (intercellular fluid) surrounded by an insulated housing (cellular membrane). By approximating each segment of a dendrite as an equivalent cylinder, or segment of cable, each cylinder becomes its own mathematical compartment. In order for current to leave or enter a compartment it must either pass through the walls of the compartment (which represent the cell membrane), or flow to another compartment (representative of current flowing to other segments of dendrite). In keeping with the original cable model developed for telegraphs, the passive diffusion of current down the dendrite is limited to a capacitance and resistance. See figure 2.4 for a visual representation of this concept. In more complex models the membrane can be assigned Hodgkin-Huxley like characteristics (Johnston and Wu, 1999; Hines and Carnevale, 1997). This includes a variety of voltage-gated ion channels in addition to the passive components. The intercellular and extracellular fluid is typically represented as a simple resistance.

The limit of an infinite number of membrane circuits placed infinitely close together results in the general equation for the cabel model; a partial differential equation of membrane voltage as a function of time and distance. 
The classic mathematical representation of cable theory known as the cable equation is as follows,

$$
\frac{1}{r_{i}} \frac{d^{2} V_{m}}{d x^{2}}=c_{m} \frac{d V_{m}}{d t}+\frac{V_{m}}{r_{m}}
$$

where $r_{i}$ is the axial resistance of dendrite in $\Omega / \mathrm{cm}, V_{m}$ is the membrane voltage, $x$ is the distance along the cable, $c_{m}$ is the specific membrane capacitance in $\mathrm{F} / \mathrm{cm}$ and $r_{m}$ is the specific membrane resistance in $\Omega-\mathrm{cm}$.

Under passive membrane conditions, the cable equation is linear and can be solved analytically, but HH neurons and HH-like neurons have nonlinear ionic membrane currents. By approximating the cell morphology as a series of discrete membrane circuits a fixed distance apart, the modified cable equation can be solved numerically (Hines and Carnevale, 1997). By making the assumptions that axial current is defined by the voltage drop between the centres of neighbouring compartments divided by the axial resistance, and that spatially varying membrane current can be approximated by its value at the center of each compartment, the cable equation can be discretely approximated as:

$$
c_{m_{j}} \frac{d V_{m_{j}}}{d t}+i_{\text {ionic }_{j}}=\sum_{k} \frac{V_{m_{k}}-V_{m_{j}}}{r_{i_{j k}}}
$$

where $V_{m_{j}}$ and $V_{m_{k}}$ are the membrane voltages in the $j t h$ and $k t h$ compartments respectively, $c_{m_{j}}$ is the membrane capacitance of the $j t h$ compartment, and $r_{i_{j k}}$ is the axial resistance which separates compartment $j$ from compartment $k$ (Hines and Carnevale, 1997). Note that this equation does not make assumptions as to how many neighbours each compartment can have. Compartments located in the middle of a dendritic segment may have two neighbours, a proximal neighbour and a distal neighbour. However, a dendritic segment located at a branch point may have three or more neighbours. One proximal neighbour and two or more distal neighbours relating to each of the daughter branches. 
Additionally, by replacing $i_{\text {ionic }}$ with any number of active currents, such the sodium and potassium currents in the HH model, current flow can be modelled through complex dendritic morphologies with active membrane dynamics. Using this approach the cable equation can be used to examine how neurons integrate synaptic inputs, and quantitatively examine how neurons generate complex three dimensional electric fields.

\subsection{Current Dipole Moments and the Forward Projection}

The previous two sections described how neurons generate electric currents. This section examines how electric currents result in scalp potentials detectable by EEG electrodes. When mathematically characterizing electric fields in the brain, neuronal sources are often modelled as a current dipole moment (sometimes shortened to "current dipole" or simply "dipole"). Current dipoles can be conceptualized as being similar to a magnet. Most ordinary magnets have a north and a south pole. Magnetic field lines originate from the north pole and return to the magnet through the south pole. Current dipoles behave in much the same way. Each dipole has a source and a sink. Current flows from the source and into the sink. In a Pyramidal cell, excitatory synapses cause current to flow from the extracellular space into the cell, thus appearing as a current sink when viewed from the extracellular space. Inhibitory synapses cause current to flow from the cell to the extracellular space, thus acting as a source (Lopes da Silva, 2010). On a pyramidal cell, excitatory synapses can located anywhere on the dendrites, whereas inhibitory synapses are localized to the soma and basal dendrites (Spruston, 2008). As a result current flows from an inhibitory synapse on the basal dendrites and along the primary axis of the neuron before being pulled back into the cell through an excitatory synapse on the apical dendrites. 


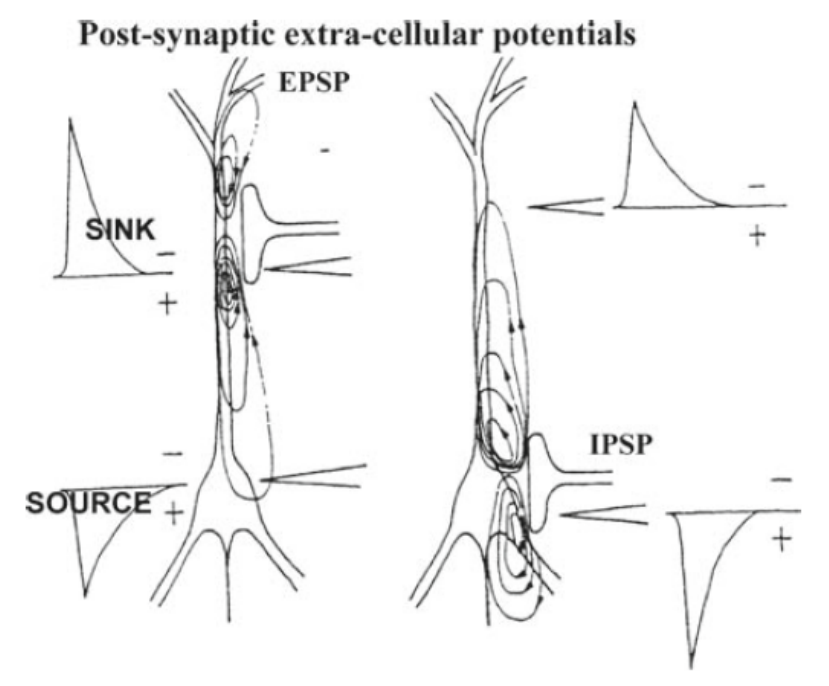

Figure 2.5: A pyramidal cell receiving two synaptic inputs. When viewing the cell from the extracellular space excitatory synapses on the apical dendrites cause current to flow into the cell and thus acts as a current sink. The inhibitory synapses on the soma causes current to flow out of the cell thus acting as a current source. Together the synapses show how a pyramidal cell can be visualized as a current dipole. Reproduced from (Lopes da Silva, 2010).

In this way a pyramidal cell can then be modelled as a single dipole (figure 2.5), where the soma and basal dendrites are modelled as the current source and the apical dendrites are modelled as the current sink (Lopes da Silva, 2010; Llinas and Nicholson, 1974). The mathematics behind how a current dipole results in a scalp potential is referred to as the forward problem. Dipoles generated by individual cells in the cortex are too small to be detected by EEG electrodes. Cells must create dipoles in unison with other cells to create a dipole powerful enough to pass through the skull and be measurable at the scalp. Additionally, the cellular dipoles must have the same or similar orientation so they spatially sum (figure 2.6).

At a macroscopic scale, a dipole can be converted to a scalp potential using a lead field model, to map $p$ electrical dipoles to $N$ electrical potentials on the 


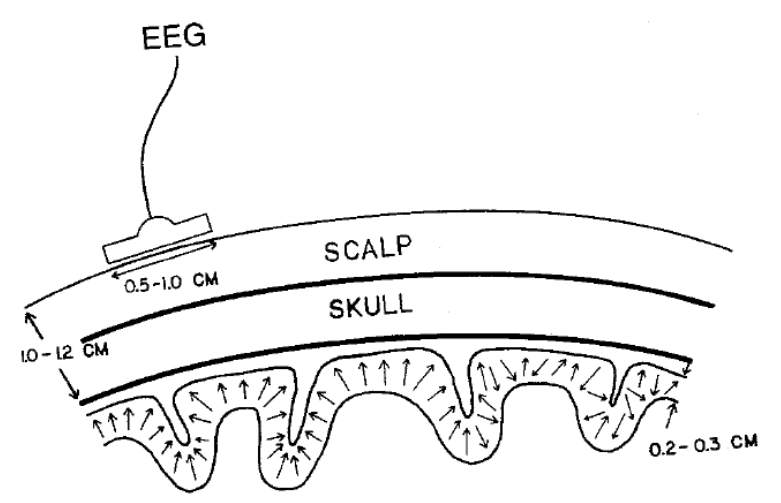

Figure 2.6: A schematic representation of how equivalent electrical dipoles, represented here as arrows in the neocortex, are located relative to the cerebral spinal fluid,the skull, the scalp, and EEG electrodes. On the left dipoles in the gyri have uniform orientations allowing for signals to add. In the sulci dipoles point in opposite directions potentially causing signals to cancel each other out. The far right of the figure shows how dipoles with random orientations can cause even signals in the gyri to be significantly weaker than when orientations are uniform. Reproduced from (Nunez and Srinivasan, 2006).

scalp. Mathematically this is represented:

$$
\left[\begin{array}{c}
V_{1} \\
\vdots \\
V_{N}
\end{array}\right]=\left[\begin{array}{ccc}
g\left(\vec{r}_{1}, \vec{r}_{d i p_{1}}, \vec{e}_{d_{1}}\right) & \ldots & g\left(\vec{r}_{1}, \vec{r}_{d i p_{p}}, \vec{e}_{d_{p}}\right) \\
\vdots & \ddots & \vdots \\
g\left(\vec{r}_{N}, \vec{r}_{d i p_{1}}, \vec{e}_{d_{1}}\right) & \ldots & g\left(\vec{r}_{N}, \vec{r}_{d i p_{p}}, \vec{e}_{d_{p}}\right)
\end{array}\right]\left[\begin{array}{c}
d_{1} \\
\vdots \\
d_{p}
\end{array}\right]
$$

or in vector form

$$
\mathbf{V}=\mathbf{G D}+\mathbf{n}
$$

Where, $\mathbf{V}$ is an $N x 1$ vector, $\mathbf{D}$ is a $p x 1$ vector of dipoles, $\mathbf{G}$ is the lead field matrix with dimensions $N x p$, and $\mathbf{n}$ is a noise matrix with dimensions $N x 1$. Each element of the lead field matrix is a function which calculates the $i_{t h}$ dipole's effect on the $j_{t h}$ scalp potential. The lead field is a function of the vector location of the scalp potential being measured $\vec{r}$, dipole location $\vec{r}_{d i p}$, and dipole orientation $\vec{e}_{d}$ (Hallez et al., 2007). The actual form of the lead field changes 


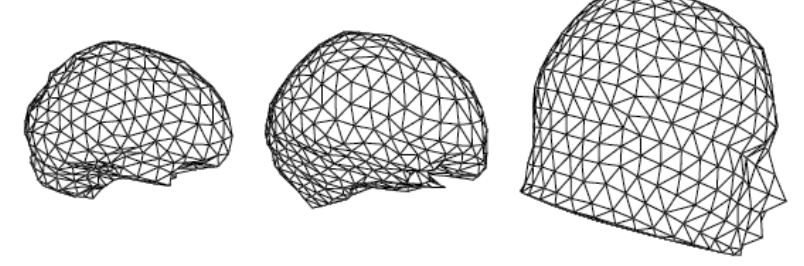

Figure 2.7: An example Boundary element model. Left: 3D reconstructed boundary between the brain and the skull. Middle: The boundary between the skull and the scalp. Right: The boundary between the scalp and the air. Reproduced from (Hallez et al., 2007).

based on the method used to solve the forward problem. The simplest approach, called the three-sphere model, approximates the brain, skull, and scalp as three concentric spheres of increasing diameter. Each sphere is assigned its own properties such as tissue permittivity. The boundary element method (BEM) for solving the forward problem incorporates patient specific geometries acquired via magnetic resonance imaging $(\mathrm{MRI})$ to determine the size and shape of the brain, skull, and scalp (figure 2.7). This method also assumes that tissue permittivity within each volume is constant and only changes at the boundary Hallez et al., 2007).

In subsections 2.1 and 2.2 we looked at how to mathematically characterize dipoles from membrane to whole cell. Using NEURON simulation software (Carnevale and Hines, 2006), Murakami and Okada simulated four different three dimensional neurons in a situation where a direct injection of current into their somas led to the generation of an action potential. They then characterized the current dipoles generated by the spiking neurons. Their results showed that 50,000 neurons generating the dipoles characterized in their simulations could produce signals detectable on the scalp. However, they did not test two specific things. First they did not look at the dipole moments generated by synaptic 
activity on the cells. Second, they did not actually simulate the signals that could be generated by networks of the three dimensional neurons. Aim 1 specifically looks to characterize the current dipole moments for both action potentials as well as post synaptic potentials. Additionally, it looks to more fully characterize how action potentials contribute to population dipoles. Aim 2 examines how networks of neurons generate population dipoles large enough to be detectable at the scalp. Aim 3 identifies the primary contributing factors to the population dipoles.

\subsection{Network Dynamics}

\subsubsection{Single Compartment Neuron Models}

Knowing how a single neuron can generate an electric field that extends to the scalp is important, but the field generated by a single neuron is undetectable by EEG electrodes (Nunez and Srinivasan, 2006; Lopes da Silva, 2010, Olejniczak, 2006). In order to understand what signals are detected by EEG electrodes it is also necessary to understand how they arise within dynamics of a populations of interacting neurons. This is where the Hodgkin-Huxley model has one of its most important corollaries. If we assume that the patch of membrane being simulated by the $\mathrm{HH}$ equations forms a bounded sphere, we can use the $\mathrm{HH}$ model as a representation of an entire neuron, or more specifically of a neuronal soma. If we assume the current $I$, in the HH equation (eqn. 2.12) to be input to the soma from dendritic (synaptic) sources, then the entirety of a neuron can be simulated as a single set of differential equations. This type of neuron is known as a point source or single compartment neuron (Dayan and Abbot, 2001).

In order to investigate the how biological factors can impact network 
dynamics $\mathrm{HH}$ or HH-like neurons must be used. HH-like neurons are single or dual compartment neurons which have dynamics similar to an $\mathrm{HH}$ neuron, but with additional ion channels (Hodgkin and Huxley, 1952; Dayan and Abbot, 2001; Eliasmith and Anderson, 2003). The channels can be voltage-gated, governed by concentrations of specific ions, or even g-protein coupled channels. Some of these neurons are single compartment neurons, while other, more complicated, neurons have a dendritic compartment and an axio-somatic compartment. The separate compartments can have distinct ionic currents which can help determine how specific neurons integrate synaptic inputs. Additionally, the ratio of the surface areas of the dendritic and axio-somatic compartments can effect the firing characteristics of the neuron. The drawback being that the increased realism in the model comes at the cost of increased computational load.

\subsubsection{Networks of Point Source Neurons}

Point source neuron models are most useful when organized into populations. The neurons in the populations are then connected via model synapses to other neurons to represent specific neural networks (Herzfeld, 2011; Eliasmith and Anderson, 2003; Bazenhov et al., 2002). These synaptic connections can either be to other neurons within the same population, known as recurrent connections, or to neurons in other populations, known as feedforward or feedback connections (Dayan and Abbot, 2001). The strength of a synaptic connection, or its ability to depolarize the somatic membrane, is determined by several factors. As discussed in section 2.2, the farther away from the soma a synapse is, the more the signal decays before reaching the soma. Additionally, factors such as receptor density at the synaptic site and neurotransmitter concentration in the synaptic cleft, also contribute to the magnitude of the 
depolarization of the soma. All of these complexities can be wrapped up into a scaling factor known as a synaptic weight, which scales the post synaptic response of a neuron. Generically, weighting can be described as:

$$
I_{s}(t)=w K_{s}(t)
$$

where $I_{s}(t)$ is the weighted post synaptic current, $w$ is the synaptic weight, and $K_{s}(t)$ is the synaptic kernel, which is a generic placeholder for any number of synaptic models (see section 2.1.2). For a neuron receiving multiple synaptic inputs from $N$ neurons, the synaptic weights can be organized into a single weight vector $w$, where $w_{b}$, is the $b_{t h}$ input neuron's synaptic weight then the total synaptic current $I_{s}$ at time $t$ can be represented as

$$
I_{s}=\sum_{b=1}^{N} w_{b} \int_{-\infty}^{t} d \tau K_{s}(t-\tau) \rho_{b}(\tau)
$$

where $\tau$ is the timing of an synaptic potential, and the expression $\rho_{b}$ is the neural response function described by the equation

$$
\rho_{b}=\sum_{i} \delta\left(\tau-t_{i}\right)
$$

Here, $t_{i}$ is the timing on a sequence of presynaptic spikes from the input neuron $b$ and $\delta$ is the delta function. This can be used to determine the membrane voltage of a given neuron in a simulation, and can be repeated for every neuron in a population and every population in a simulation to determine the membrane voltages and spiking dynamics of the network. 


\section{Characterizing Cellular Dipoles}

Although there are many techniques for analysing EEG signals, none directly elucidate the underlying network activity. At the electrode level, time-frequency analysis associates increases and decreases in power of certain frequency bands over time with behavioural measures. Coherence analysis looks at the functional connectivity of one region of the brain with another. Source localization attempts to reconstruct the magnitudes of the current dipoles associated with the generation of EEG signals. These dipoles can then be analysed in much the same way as the EEG signals, using time-frequency analysis and coherence measures. However, it remains unclear how the electrical activity in specific populations of neurons relates to EEG activity measured at the scalp. The primary reason for this is that the a direct relationship between neural activity and a current dipole moments has not been fully explored.

Here we develop a series of computational simulations to investigate the relationship between individual neural activity, the equivalent population level current dipole, and the measured EEG signal. The first set of simulations will use detailed three dimensional simulations of neurons to investigate how neurons generate dipoles in response to different types of activity (spiking or synaptic).

\subsection{Cell Models}

Simulations of individual neurons were conducted using the NEURON simulation environment (Carnevale and Hines, 2006). Two neuron models, developed by Drs. Zachary Mainen and Terrence Sejnowski Mainen and Sejnowski, 1996), were selected from the NEURON database (McDougal et al. 2017) (obtained from ModelDB accession number 2488), an excitatory Pyramidal 
cell and an inhibitory spiny stellate cell. The model pyramidal cell was a digital reconstruction of a layer $\mathrm{V}$ pyramidal cell located in the cortex of a cat (figure 3.1a). The generic interneuron used was a digital reconstruction of a layer IV spiny stellate cell from the somatosensory cortex of a rat (figure $3.1 \mathrm{~b}$ ).

\section{Pyramidal Cell}

The pyramidal cell consists of 479 cylindrical compartments of varying diameter. To ensure numerical accuracy of the simulations, compartment length was limited to a maximum of $50 \mu \mathrm{m}$. All compartments contained a passive membrane resistance and a passive membrane capacitance. The dendritic compartments contained ion channels for four ionic currents: a fast activating sodium current, a slow non-inactivating potassium current, a calcium current, and a calcium dependent potassium current. In addition to the four ionic currents found in the dendrites, the soma included a delayed rectifying potassium current. The hilloc of the pyramidal cell contained only the traditional Hodgkin-Huxley currents; a fast activating potassium and a delayed rectifying potassium current, whose maximum conductances were approximately four orders of magnitude greater than those in the soma and dendrites. Equations and parameter values used for each of the ionic currents are provided in appendix A.1. The particular pyramidal cell used was a spiny cell, meaning that the dendrites are covered in microscopic spines. Computationally it becomes too difficult to simulate each spine as its own compartment, which would add thousands of compartments to each neuron in the simulation. To model the spines without increasing computational load, the membrane surface properties were scaled by the following factor:

$$
F_{k}=\left(L_{k} * S A_{\text {spine }} * D_{\text {spine }}+A_{k}\right) / A_{k}
$$




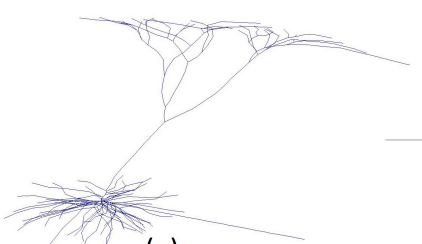

(a)

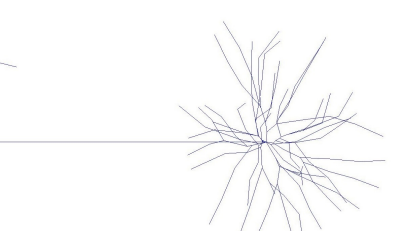

(b)

Figure 3.1: Three dimensional NEURON models used. (a) A representation of the layer $\mathrm{V}$ pyramidal cell used for dipole moment calculations. The tuft of dendrites at the bottom are the basal dendrites, with the axon protruding to the right. The dendrites stretching out above the basal dendrites are the apical dendrites. (b) A representation of the layer IV spiny stellate cell used for the dipole moment calculations. The cell consists of a tuft of dendrites with the axon protruding out to the left. The original models were downloaded from ModelDB (McDougal et al., 2017) (accession number 2488).

where $L_{k}$ is the length of the kth compartment within a dendritic branch, $S A_{\text {spine }}$ is the surface area of a single spine, $D_{\text {spine }}$ is the density of spines per $\mu m$, and $A_{k}$ is the area of a given dendritic branch.

\section{Regularly Spiking Pyramidal Cell}

The dynamics of the ionic currents in combination with the dendritic morphology of the original pyramidal cell model caused the cell to fire in a chattering pattern (neurons which fire in clusters of action potentials). However, not all pyramidal cells exhibit bursting behaviour; known as chattering (Mainen and Sejnowski, 1996; Izhikevich, 2003). In order to explore the dipoles created by both chattering pyramidal cells and regularly spiking pyramidal cells, the calcium and calcium dependent potassium currents were removed from all segments of the neuron to create regularly spiking activity (single action potentials).

\section{Interneuron}

The brain contains several hundred different types of neurons Guyton and Hall, 1996). As a first step toward characterizing the contributions of inhibitory 
interneurons we chose a single neuron model to be representative of all interneuron activity. The spiny stellate cell was chosen because it is one of the most common interneurons in the brain (Riera et al., 2012). The NEURON model consists of 199 compartments; each restricted to be no greater than $50 \mu \mathrm{m}$ in length. Ionic currents were assigned to the spiny stellate cell in the same manner as the pyramidal cell. Dendritic compartments contained a fast activating sodium current, a slow non-inactivating potassium current, a calcium current, and a calcium dependent potassium current. The soma included the above currents with the addition of a delayed rectifying potassium current. The hilloc contained only the traditional Hodgkin-Huxley currents with maximum conductances of approximately 4 orders of magnitude greater than those in the soma and dendrites. Like the pyramidal cell model, spines were added to each of the dendritic compartments by scaling of the membrane capacitance and maximum conductances.

\subsubsection{Calculation of Current Dipole Moment}

The methodology for calculating the current dipoles is a modified version of method found in Murakami and Okada (2006). For each simulation, the current dipole was estimated using one of the three dimensional NEURON models discussed above. Each compartment was considered to have its own current

dipole $\vec{Q}_{k}$, denoting the vector quantity of the current dipole $\vec{Q}$ and its orientation in the $\mathrm{x}, \mathrm{y}$, and $\mathrm{z}$, axes, in the $k_{t h}$ compartment. $\vec{Q}_{k}$ is calculated by the following formula;

$$
\vec{Q}_{k}=I L_{k} d \vec{r}
$$

Where $I_{k}$ is the longitudinal current in the compartment, $L_{k}$ is the length of the compartment and $d \vec{r}$ is the compartments unit direction vector. The longitudinal 
current I can be computed as follows;

$$
I_{k}=\frac{-\pi * a_{k}^{2}}{\rho_{L}} \frac{\partial v}{\partial x}
$$

where $a_{k}$ is the radius of the compartment, $\rho_{L}$ is the longitudinal resistivity, and $\frac{\partial v}{\partial x}$ is the partial derivative of voltage with respect to length along the dendrite. The partial derivative of voltage was numerically approximated using the finite voltage difference, $\partial v$, calculated between the voltage at the beginning, $v_{0}$, and end, $v_{1}$, of each compartment. The finite position difference, $\partial x$, was equal to the length of the compartment. Thus the partial derivative of voltage with respect to time, $\frac{d v}{d t}$, became $\frac{v_{1}-v_{0}}{L}$. The current dipole of the entire cell was calculated by taking a vector sum of dipoles across all of compartments. Finally, since EEG electrodes are located on the scalp only dipole contributions pointed in the direction of the electrodes were considered. Therefore, the dot product was taken between the cellular current dipole vector, $\vec{Q}$, and the unit vector perpendicular to the pial surface, $d \vec{r}$. For pyramidal cells the unit vector perpendicular to the pial surface is defined as being approximately parallel to the apical dendrite. Using this approach the complex geometry and electrophysiology as reduced to a single time series. We refer to this time series as a Dipole Response Function (DRF).

\section{Spiking Dipole Response Functions}

To calculate the current dipole moment associated with neural spiking activity, a current was injected directly into the soma of the cell via the NEURON IClamp function. For both chattering and regularly spiking pyramidal cell models a current of $0.12 \mathrm{nA}$ was injected for $400 \mathrm{~ms}$. For the spiny stellate cell model a current of $0.07 \mathrm{nA}$ was injected into the soma for a duration of $400 \mathrm{~ms}$. The current amplitude were selected to be large enough to induce a spike yet 
small enough to allow for the current dipole to be cleanly epoched on either side of the spike. This second portion is important as the method of convolution used in aim 3 creates a temporal artefact if all the input DRFs are not of the same length.

This artefact comes from the fact that the convolution is an integral is used to multiply two functions and is normally solved analytically from $-\infty$ to $\infty$. However, when using the matlab conv function the convolution is calculated using two discrete arrays of finite length, arrays A and B. The length of the output array, C, is length(A)+length(B)-1. Thus if the DRFs, (e.g. array A) are different lengths they will produce output arrays, C, which are different lengths. Additionally, since we want the output array, C, to be the same length as the input spike train, say length(B), the 'same' option was used. This gives the central $\max ($ length(A),length(B)) points from array C as the output. Since only the right side of the input arrays are padded, taking the central points for arrays of different lengths shifts the apparent location of events in the shorter arrays left, relative to the longer arrays.

\section{Dipole Response Functions from Post Synaptic Potentials}

Creating a separate DRF for every synapse location on a neuron become computationally infeasible for networks with thousands of neurons and tens of thousands of synapses. To create a more scalable method of simulating synaptic DRFs a generic spatial average was created. For each cell model a DRF was created for a generic excitatory post synaptic potential (EPSP) and a generic inhibitory post synaptic potential (IPSP). To simulate synapses, the NEURON function exp2syn was used, which uses the difference of exponential model to calculate the conductance change in the membrane. The time constant for the rising exponential was set to $1 \mathrm{~ms}$, and the time constant for the decaying 
exponential was set to $6 \mathrm{~ms}$ (Gerstner et al., 2014; Destexhe and Par, 1999). The maximum conductance for the synapse was set to $6.5 \mu \Omega$ for excitatory synapses and $-6.5 \mu \Omega$ for inhibitory synapses. The spatial average DRF was created by running a simulation where a single synapse was placed on a single dendritic compartment and the resulting DRF was calculated. The synapse was then moved to another dendritic compartment and the process was repeated for every dendrite in a specific subset of the dendritic tree which varied for each synaptic type. The DRFs from each individual simulation were averaged. This allowed for the creation of a generic response from a synapse occurring anywhere within specific subset.

For pyramidal cells in the cortex inhibitory synapses are spatially localized to the basal dendrites. Therefore, the spatial average DRF for an IPSP on a pyramidal cell was obtained by averaging across the basal dendritic compartments. According to the open field theory, the apical dendrites of a pyramidal cell are responsible for creating the current sink. Therefore when calculating the spatial average DRF for an EPSP on a pyramidal cell, the locations of the synapses were restricted to the apical dendrites. Finally, since spiny stellate cells have no documented synaptic organization, the entire dendritic tree was used to create the spatial average DRF for the EPSP. There were no inhibitory synapses on the interneurons in aims 2 and 3 so no IPSP was generated for the spiny stellate cell.

\subsection{Dipole Response Functions}

We used three separate neuron models. Two of the models (chattering pyramidal cell and spiny stellate cell) were used taken directly from Mainen and Sejnowski (1996). Firing characteristics for these unaltered cell models match 

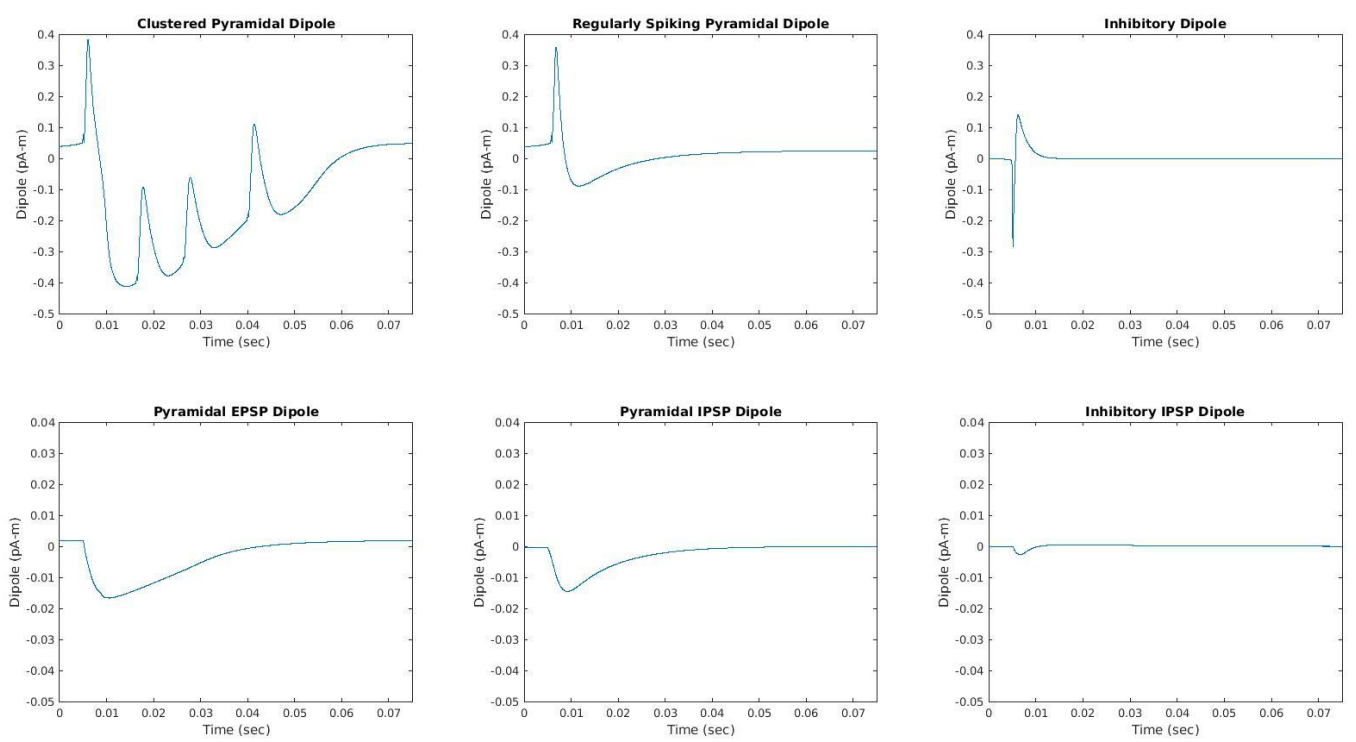

Figure 3.2: Dipole Response Functions. Top row shows DRFs in response to an action potentials generated through direct injection of current to the soma of the cell. (Top left) Shows DRF for chattering pyramidal cell. Note that the width of the spikes are much wider $(8-10 \mathrm{~ms})$ than what would be seen for a voltage trace of an action potential (1-2 ms). (Top middle) Shows DRF for Regularly spiking Pyramidal cell. Also note the duration of the spike is $\sim 3 \mathrm{~ms}$. (Top right) Shows spiking spiny stellate cell. Has an amplitude similar to that of the regularly spiking pyramidal cell, however, the duration is much shorter $<1 \mathrm{~ms}$. The bottom row shows DRFs from post synaptic potentials. Note the change in scale on the $y$ axis from the top row to the bottom row. (Bottom left) DRF for an EPSP on a pyramidal cell. This DRF represents an average of dipoles recorded from multiple locations on the apical dendrites (see sec 3.1.1 for more details). (Bottom middle) IPSP on a pyramidal cell. This DRF represents an average of dipoles recorded from multiple locations on the basal dendrites. (Bottom right) DRF for an EPSP on a spiny stellate cell. This DRF represents an average of dipoles recorded from multiple locations on the dendritic tree. 
those reported in Mainen and Sejnowski (1996). The dipoles were also similar to those found in Murakami and Okada (2006). The third model (the regularly spiking pyramidal cell) was made by altering the chattering pyramidal cell model. Graphs of all DRFs can be found in figure 3.2 .

For the chattering pyramidal cell each individual action potential created a corresponding spike in the DRF, but also had an envelope of activity. The spikes had an amplitude of approximately $0.32 \mathrm{pA}-\mathrm{m}$. While the amplitude of the envelope was $-0.41 \mathrm{pA}-\mathrm{m}$. The duration of each spike was approximately 8-10 ms, which is considerably longer than the action potential $(\sim 1-2 \mathrm{~ms})$. The duration of the envelope was approximately $50 \mathrm{~ms}$ (determined as the time from the onset of the first spike to the time the envelope decays to $5 \%$ of its peak value). For the regularly spiking pyramidal cell, the magnitude of the spike was approximately $0.325 \mathrm{pA}-\mathrm{m}$, and the afterhyperpolarization had a magnitude of $0.1 \mathrm{pA}-\mathrm{m}$. For the spiny stellate cell the initial spike had an amplitude of 0.27 pA-m and an afterhyperpolarization of $0.14 \mathrm{pA}-\mathrm{m}$. The duration of the initial spike was $0.8 \mathrm{~ms}$ for the spiny stellate cell while for the pyramidal cell it was 3 ms. The afterhyperpolarization on the spiny stellate had a duration of $5.6 \mathrm{~ms}$ while in the pyramidal cell it lasted approximately $18 \mathrm{~ms}$.

For the DRFs related to post synaptic potentials, the magnitude of the EPSP on the pyramidal cell was $0.0217 \mathrm{pA}-\mathrm{m}$, while the magnitude of the IPSP on the pyramidal cell was $0.0290 \mathrm{pA}-\mathrm{m}$. The duration of the EPSP was approximately $36 \mathrm{~ms}$ long (determined as the time from onset until the PSP decays to $5 \%$ of its peak value), while the duration of the IPSP was approximately $42 \mathrm{~ms}$ long. For the EPSP on a spiny stellate cell the magnitude was $-0.0017 \mathrm{pA}-\mathrm{m}$. The duration of the PSP is relatively short, $5 \mathrm{~ms}$, however, it has an afterhyperpolarization which is has an amplitude of $0.0003 \mathrm{pA}-\mathrm{m}$ but a 


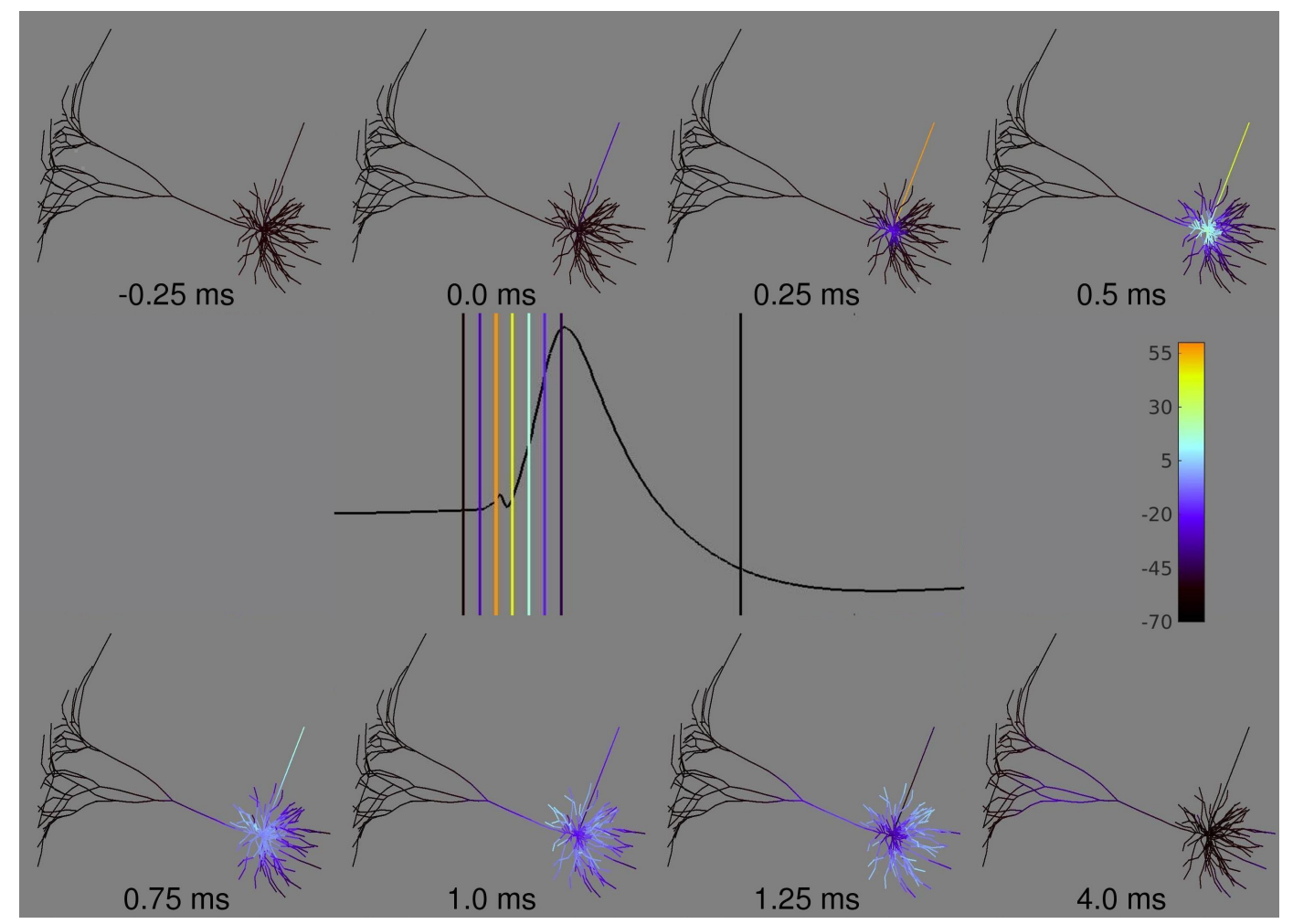

Figure 3.3: Time-lapse of membrane voltage for a regularly spiking pyramidal cell during an action potential. The top and bottom rows shows the spatial distribution of membrane voltage across the cell's dendritic tree. Changes in membrane potential are coloured relative to resting potential. Black segments are at resting potential $(\sim-65 \mathrm{mV})$ and yellow segments are at peak voltage $(\sim 60 \mathrm{mV})$. The middle plot shows the DRF for the regularly spiking pyramidal cell shown seen in fig. 3.2. The vertical lines indicate where the individual frames of the time-lapse are taken from. The color of each bar relates to the color of the axon during the given frame for easier reference. Time stamps on the bottom of each frame are referenced from the initiation of the action potential. 


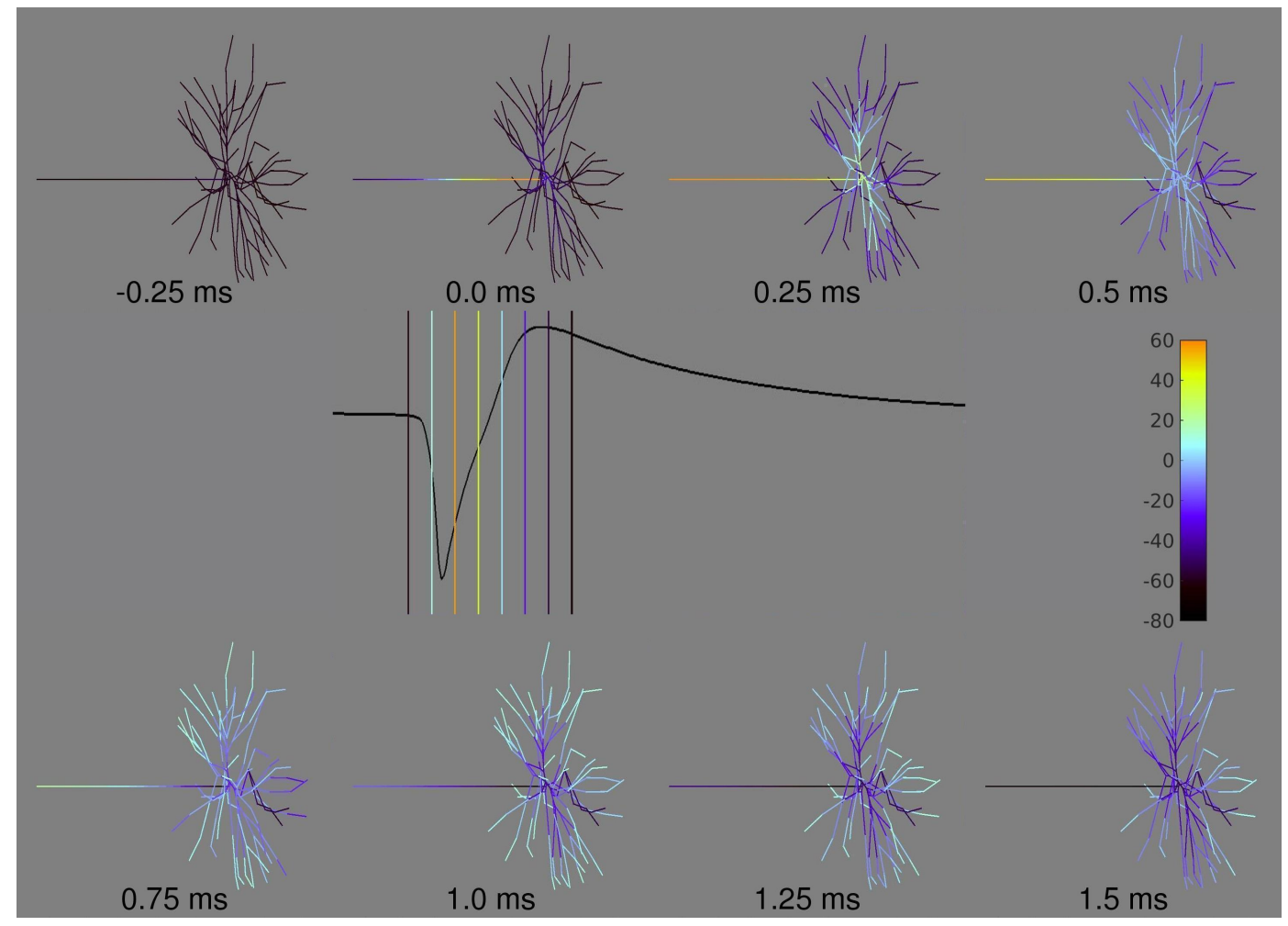

Figure 3.4: Time-lapse of membrane voltage for a spiny stellate cell during an action potential. The top and bottom rows shows the spatial distribution of membrane voltage across the cell's dendritic tree. Changes in membrane potential are colored relative to resting potential. Black segments are at resting potential $(\sim-75$ $\mathrm{mV})$ and yellow segments are at peak voltage $(\sim 60 \mathrm{mV})$. The middle plot shows the DRF for the regularly spiking pyramidal cell shown seen in fig. 3.2. The vertical lines indicate where the individual frames of the time-lapse are taken from. The color of each bar relates to the color of the mid point of the axon during the given frame for easier reference. Time stamps on the bottom of each frame are referenced from the initiation of the action potential. 


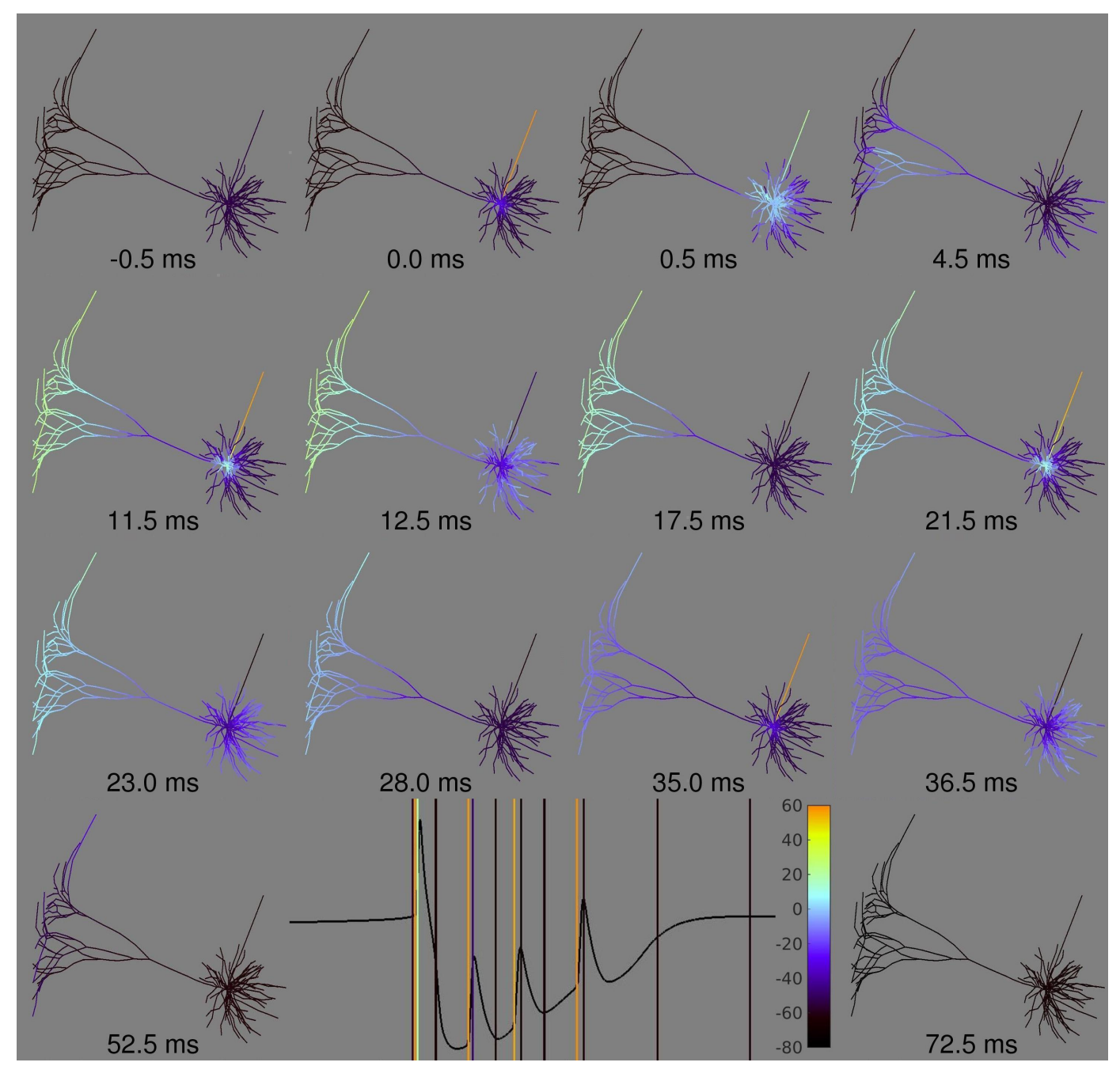

Figure 3.5: Time-lapse of membrane voltage for a chattering pyramidal cell during an action potential. The smaller plots of the pyramidal cells show the spatial distribution of membrane voltage across the cell's dendritic tree. Changes in membrane potential are colored relative to resting potential. Black segments are at resting potential $(\sim-80 \mathrm{mV})$ and yellow segments are at peak voltage $(\sim 60 \mathrm{mV})$. The bottom center plot shows the DRF for the chattering pyramidal cell as seen in fig. 3.2 . Vertical lines indicate where the individual frames are located on the DRF. The color of each bar relates to the color of the axon during the given frame for easier reference. Times are referenced to the initiation of the initial action potential. 
duration of $35 \mathrm{~ms}$.

Though the regular spiking pyramidal cell and the spiny stellate cell have similarly shaped DRFs, the main difference is that Pyramidal cells response is longer. This is because the spike related DRFs are not generated directly by the action potential itself (defined as propagation of current along the axon due to positive feedback of sodium currents), but rather to back propagation of currents up the dendritic tree due to an excess of current generated at the axonal hilloc during an action potential. Since the pyramidal cell has a larger and more complex dendritic tree, it takes longer for the current to propagate along the dendrites causing the DRF to have a longer response. The backpropagation effect is best illustrated in figures $3.3,3.4$ and 3.5 . Figure 3.3 shows the membrane voltages of each dendtritic compartment for a regularly spiking pyramidal cell in snapshots across time. The action potential can be seen forming in the axon at the $0 \mathrm{~ms}$ snapshot. Over the next few snapshots the action potential can be seen propagating the length of the axon until it dissipates around $1 \mathrm{~ms}$ after its generation. At the same time you can see current propagate from the soma and axonal hilloc into the dendritic tree. The current dipole moment reaches peak magnitude after approximately $1.25 \mathrm{~ms}$, as illustrated by the current dipole trace in the center of the figure. The current dipole generated by the back propagating current still has a measurable effect until at least $2.5 \mathrm{~ms}$ after the initial generation of the action potential, and doesn't cross zero until after $3 \mathrm{~ms}$. This figure illustrates why the spike in the DRF lasts longer than the action potential. This is contrasted by figure 3.4 which shows the backpropagation of current in a spiny stellate cell. The figure illustrates how the action potential lasts longer than the initial spike in the DRF. The dendrites on spiny stellate are much shorter and it does not take as long for the current to back propagate. 

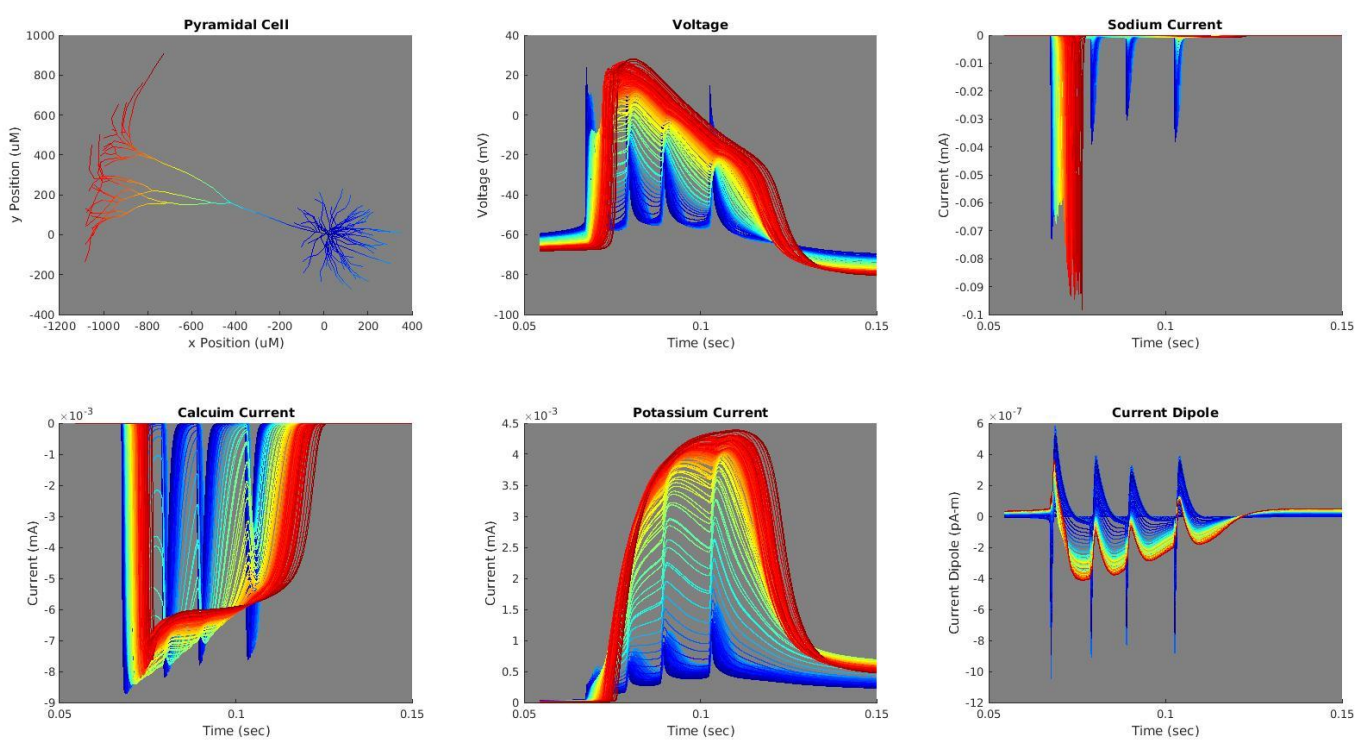

Figure 3.6: Compartmental look at neuronal variables for chattering pyramidal cell. This figure shows how variables vary within individual compartments of a chattering pyramidal cell. (Upper left) Shows the chattering pyramidal cell sans axon. The color of each dendrite corresponds with the color of each trace in the corresponding plots. This allows for each trace to be related to its location on the cell. (Upper middle) Shows voltages in each compartment. Proximal dendrites have traces which have individual spikes without an envelope. Distal dendrites show an envelope without individual spikes. (Upper right) Shows sodium current. These plots show a distinct spike all the way up the dendritic tree for the initial spike, however, subsequent action potentials only yield a noticeable sodium current in the proximal dendrites (Bottom left) Shows calcium current. In the proximal dendrites there are distinct spikes with no envelope. In the distal dendrites there is a distinct envelope with no spikes. (Bottom Middle) Shows potassium current. In the proximal dendrites there are small spikes on a low amplitude envelope. Distal dendrites have a large amplitude envelope and low amplitude or missing spikes.(Bottom Left) Shows the summation of individual dipoles from the most proximal dendrites to the most distal dendrites. For this plot each line shows the summation of the that dendritic compartment with every compartment which is more proximal to the soma. The most proximal compartments show distinct spikes in their dipoles. Moving distally along the dendrites tends to create and deepen the envelope to the dipole. 
The impact of backpropagating currents on the DRF is even more pronounced for a chattering pyramidal cell (figure 3.5). After the generation of the initial action potential at $0 \mathrm{~ms}$ the current propagates up the dendritic tree. Additional action potentials occurred at 11.5, 21.5, and $35 \mathrm{~ms}$, which is reflected by corresponding spikes in the DRF. The figure also illustrates the source of the envelope in the spike related DRF. After the backpropagation of current from the initial action potential faded, there was a persistent depolarization in the apical dendrites (see timepoint $4.5 \mathrm{~ms}$ ), which lasted for the length of the DRF (see timepoints $4.5 \mathrm{~ms}$ through $52.5 \mathrm{~ms}$ )

The contributions to the persistent depolarization are shown in figure 3.6 . The upper left hand plot shows a schematic of the pyramidal cell without the axonal compartments. The color of each dendrite corresponds with the color of each trace in the subsequent plots. The upper middle plot shows the voltage traces in each compartment. Membrane voltages in the dendrites proximal to the soma (blue traces) clearly show each spike in the chattering response, whereas in the distal apical dendrites there was a persistent depolarization, with no spikes.

This persistent depolarization can be explained by the interactions between dendritic radius, sodium currents, calcium currents, and calcium dependent potassium currents. First, the sodium current (seen in the upper right of figure 3.6) shows a distinct spike all the way up the dendritic tree for the initial spike that decays as until it progresses half way up the apical dendrites. After that, the sodium current grows as it reaches the most distal dendrites. This is most likely due to the decrease in dendritic radius with distance from the soma. The decreased compartment radius results in decreased surface area and decreased volume. For each cylindrical compartment the volume decreased with the square of the radius, while the surface area decreased proportional to the radius. This 


\begin{tabular}{|c|c|c|}
\hline Number of synapses & Conductance $(\mu S)$ & Min. Dipole $(\mathrm{pA}-\mathrm{m})$ \\
\hline 2 & 45 & -0.1882 \\
\hline 3 & 38 & -0.2177 \\
\hline 4 & 23 & -0.1975 \\
\hline 5 & 20 & -0.2208 \\
\hline 6 & 14 & -0.2316 \\
\hline 7 & 12 & -0.2149 \\
\hline 8 & 10 & -0.1793 \\
\hline 9 & 9 & -0.1960 \\
\hline 10 & 7.8 & -0.2303 \\
\hline
\end{tabular}

Table 3.1: Synaptic conductances and corresponding dipoles for excitatory post synaptic potentials.

increased the surface area to volume ratio increased activation of sodium channels.

\subsubsection{Comparing spiking and synaptic contributions}

Characterizing the relative contributions of PSPs to spike related dipoles can be challenging, due to the generic weighting used to generate the PSP related dipoles. In aims 2 and 3 we attempt to address this issue by normalizing the DRFs from PSPs to the weighting used in the network activity. This allows for a comparison of relative contributions of PSP to spike related dipole activity in specific networks during specific events, but it does not address the issue of generically comparing relative dipole activities of PSPs to spike related activity. To this end we conducted an additional series of simulations on the chattering pyramidal cell model to determine the dipole generated by excitatory post synaptic potentials that will result in a somatic membrane voltage just shy of threshold. We conducted 9 simulations with varying numbers of synapses located on the apical dendrites. The number of synapses ranged from 2 to 10 . The synaptic conductances used for each simulation are shown in table 3.1. Synapses 


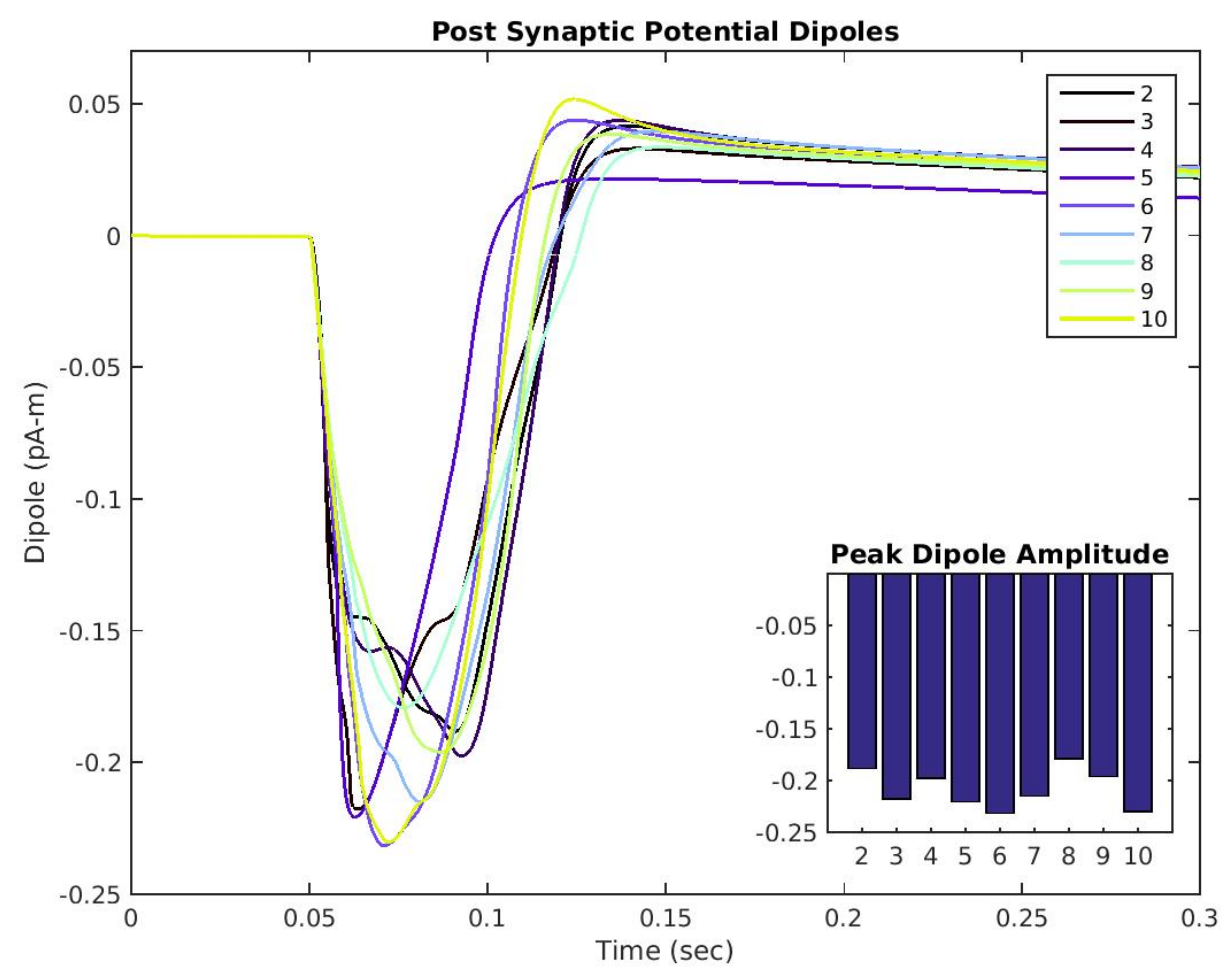

Figure 3.7: Current dipoles generated for varying numbers of synapses located on the apical dendrites at near threshold levels. The bar graph shows the peak amplitude dipole for each simulation.

were located on the most apical reaches of the dendritic tree to ensure the largest dipole possible.

The simulations show a remarkably consistent dipole magnitude with a mean of $0.2085 \pm 0.0188 p A-m$. Traces of the dipole responses are shown in figure 3.7. Individual maximums are shown in table 3.1. The mean value obtained from these simulations was half the magnitude of the envelope for the spike related dipole of the chattering pyramidal cell (0.41 pA-m). Additionally, the regularly spiking pyramidal cell had a spiking amplitude of $0.32 \mathrm{pA}-\mathrm{m}$ which was $\sim 53 \%$ larger than the average dipole generated by synaptic potentials. 


\subsection{Discussion}

Post synaptic potentials are considered to be the leading contributor to EEG signals because they last considerably longer than action potentials (Lopes da Silva, 2010; Olejniczak, 2006); 10-30 ms vs 1-2 ms respectively.

However, our simulations show that the duration of the spike related dipole can last considerably longer. The spike related dipole of an individual action potential for a chattering pyramidal cell lasted closer to $8 \mathrm{~ms}$ while the envelope lasted over $50 \mathrm{~ms}$. The duration of the envelope is longer than that of the typical post synaptic potential providing the opportunity for spike related dipole activity to sum.

For regularly spiking pyramidal cells, spike related current dipoles lasted over $3 \mathrm{~ms}$. The larger duration, relative to the action potential was driven by the backpropagation of current from the axonal hillock into the dendrites. Because dendrites are unmyelinated, the current takes longer to propagate resulting in a longer lasting current dipole . The $3 \mathrm{~ms}$ dipole duration of a regular pyramidal cell is considerably smaller than what is seen for a chattering cell. However, as few as 7 regularly spiking neurons firing asynchronously at $50 \mathrm{~Hz}$ for one second would still yield s $50 \mathrm{~ms}$ interval where two or more dipoles overlap. Murakami and Okada (2006) suggest that it would take approximately 50,000 neurons to generate a detectable signal. If that number of neurons were firing at $50 \mathrm{~Hz}$, there would necessarily be a significant amount of overlap.

Additionally, a studies by Marsalek et al. shows that cortical networks can achieve and maintain a high degree $(\sim 1 \mathrm{~ms})$ of synchronicity Marsalek et al., 1997; Abeles, 1982, 1991). These studies also showed that output jitter (i.e. the temporal variability in neuronal responses) decreases as information is passed 
from one neuronal population to another. This is due to the fact that the output jitter is almost always smaller than the input jitter (i.e. timing of PSPs)(Marsalek et al., 1997). This phenomenon has been observed in the visual cortex (Marsalek et al., 1997; Abeles, 1982). Marsalek et al. (1997) investigated this property mathematically. They derived an equation for the variance in output jitter as a function of the input jitter.

$$
\sigma_{\text {out }}=\sigma_{\text {in }} 2 \sqrt{3} \sqrt{\frac{\left(n_{\text {th }}+1\right)\left(n+1-n_{\text {th }}\right)}{(n+2)^{2}(n+3)}}
$$

where $\sigma_{\text {out }}$ and $\sigma_{\text {in }}$ are the input and output jitter respectively (measured by the standard deviation), $n_{t h}$ is the number of synapses needed to reach threshold and $n$ is the total number of synapses. For a sparsely connected network we can assume $n \gg n_{t h} \gg 1$. We can then make the approximations that $n+3 \approx n+2 \approx n+1-n_{t h} \approx n$. Substituting these approximations equation 3.4 reduces to,

$$
\sigma_{\text {out }}=\sigma_{\text {in }} 2 \frac{\sqrt{3}}{n} \sqrt{n_{t h}}
$$

In this reduced form we can see that the output jitter, $\sigma_{\text {out }}$ is directly proportional to the input jitter, $\sigma_{i n}$. However, since $\sigma_{\text {out }}$ is also inversely proportional to the number of synaptic inputs $n$, as long as $n$ is greater than $2 \sqrt{3 n_{t h}}$, output jitter will always be smaller than input jitter. Thus with each set of synaptic interactions the output jitter will continue to shrink and the neurons will become more synchronous. Marsalek et al. repeated their results using both an LIF neuron and the NEURON pyramidal cell model developed by Drs. Mainen and Sejnowski.

Additionally, a study by Stuart et al. showed that dendritic calcium currents activated by backpropigation of current due to an action potential 
(Stuart et al., 2008). It has also been shown that these calcium currents are detectable on the cortical surface (Suzuki and Larkum, 2017). This is consistent with our findings (Section 3.2), that backpropagating currents from an action potential can activate voltage dependant calcium channels, causing a persistent depolarization in the dendrites. This persistent depolarization contribution to the dipole has a magnitude and duration sufficient to generate detectable EEG signals. 


\section{Simulation of EEG Using DRFs}

\subsection{Calculation of the Population Dipole}

\subsubsection{Thalamocortical Network Model}

Aim 1 primarily focused on how individual neurons generate dipoles. This section primarily deals with aim 2 and how populations of dynamically interacting neurons generate dipoles which are detectable at the level of EEG. However, constructing a network of the morphologically accurate neurons used in aim 1 would not only lead an extremely high computational load, but the NEURON simulation software is not designed for large scale network simulations, and it would be difficult to accurately represent a large network of dynamically interacting neurons. Additionally, we need to be able to verify that the simulations are reasonably representing biologically plausible signals at multiple levels, including the underlying network activity, the dipoles being generated at the population level, and the EEG signals which result. To this end we chose to use a thalamocortical network based on the model published by Bazenhov et al. (2002). The model consisted of single and two compartment point source neurons which have a significantly lower computational load per neuron than the NEURON models and can be used to represent large networks of dynamically interacting neurons. The network is also based on data from Contreas and Steriade (1995), which contains simultaneous recordings of intercellular membrane voltages, depth EEG, and surface EEG from the somatosensory cortex and thalamus of a cat in chemically induced slow wave sleep. With this data we qualitatively and quantitatively assessed how well the model approximated the recorded data to verify that the EEG signals simulated 


\begin{tabular}{|c|c|c|c|c|}
\hline Pre & Post & Fan & Type & Max. Conductance $(\mu S)$ \\
\hline \multirow{2}{*}{ PY } & \multirow{2}{*}{ PY } & \multirow{2}{*}{ \pm 5} & AMPA & 0.15 \\
\hline & & & NMDA & 0.01 \\
\hline \multirow{2}{*}{ PY } & \multirow{2}{*}{ IN } & \multirow{2}{*}{ \pm 1} & AMPA & 0.05 \\
\hline & & & NMDA & 0.008 \\
\hline PY & $\mathrm{TC}$ & \pm 5 & AMPA & 0.025 \\
\hline $\mathrm{PY}$ & $\mathrm{RE}$ & \pm 5 & AMPA & 0.005 \\
\hline IN & PY & \pm 5 & $\mathrm{GABA}_{A}$ & 0.008 \\
\hline $\mathrm{TC}$ & $\mathrm{PY}$ & \pm 10 & AMPA & 0.1 \\
\hline $\mathrm{TC}$ & IN & \pm 2 & AMPA & 0.1 \\
\hline $\mathrm{TC}$ & $\mathrm{RE}$ & \pm 5 & AMPA & 0.4 \\
\hline \multirow{2}{*}{$\mathrm{RE}$} & \multirow{2}{*}{$\mathrm{TC}$} & \multirow{2}{*}{ \pm 5} & $\mathrm{GABA}_{A}$ & 0.2 \\
\hline & & & $\mathrm{GABA}_{B}$ & 0.04 \\
\hline $\mathrm{RE}$ & $\mathrm{RE}$ & \pm 5 & $\mathrm{GABA}_{A}$ & 0.2 \\
\hline
\end{tabular}

Table 4.1: Network topology for thalamocortical network. First column denotes the presynaptic neuron and the second column denotes the post synaptic neuron. Synaptic fan outs (number of neighbouring postsynaptic neurons the presynaptic neuron is connected to) are in the third column. Synaptic type and maximum conductances (in $\mu S$ ) are detailed in the fourth and fifth columns respectively. A detailed description of the synaptic models can be found in Appendix B

are reflective of realistic network activity.

The network consisted of four neural populations, two in the cortical layer and two in the thalamic layer. The cortical layer consisted of a pyramidal (PY) population of 100 neurons and an inhibitory (IN) population of 25 neurons. The thalamic layer consisted of a thalamocortical (TC) population and a reticular (RE) population each containing 50 neurons. Populations were arranged in arrays and connected recurrently to adjacent neurons in the same population as well as neighbouring neurons in other populations. Details of the connection topology are shown in 4.1 . The inputs to the network were spontaneous miniature EPSPs and IPSPs. These mini PSPs occurred at all AMPA mediated synapses in the cortical layer and followed the same dynamics as the synapses they originated from. The only difference was that the maximum conductance for the miniPSP was adjusted such that the magnitude of of the PSP was $\sim 0.75 \mathrm{mV}$. 


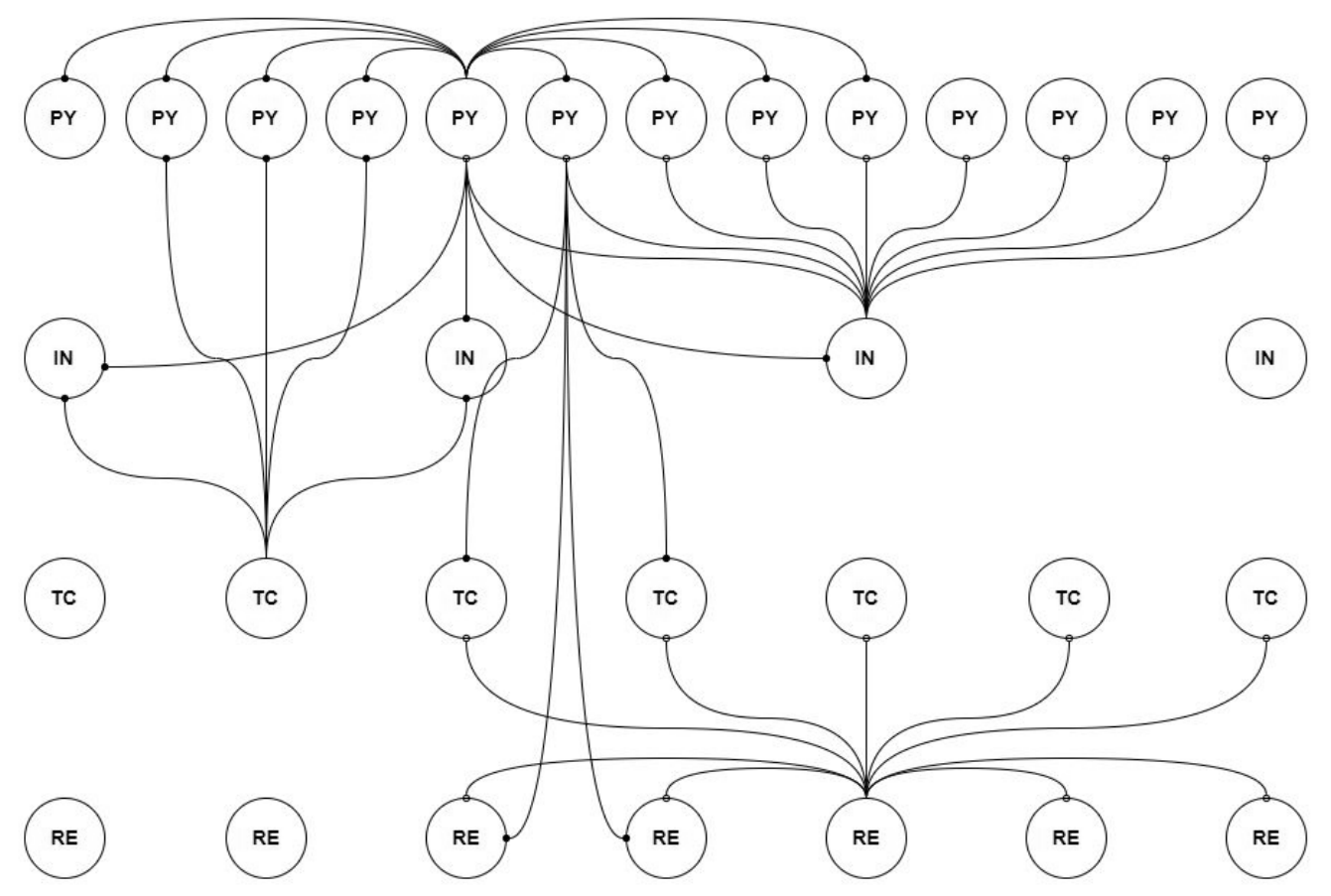

Figure 4.1: Diagram of thalamocortical network.

In contrast a single synaptic input from in a PY-PY connection necessary to achieve the seired network dynamics resulted in a depolarization just shy of threshold for an action potetial. The frequency of occurrence of mini PSPs was determined by a Poisson process with a time dependent mean rate governed by,

$$
\mu(t)=\left(\frac{2}{1+e x p^{-\frac{t-t_{o}}{\tau}}}-1\right) / 100
$$

where $\tau$ was set to 400 to produce a mean maximum rate of $10 \mathrm{~Hz}$. The time-dependant mean rate changed based on the time since the last synaptic input, $t_{o}$, with mini PSPs becoming more frequent as the time from the last spike increased. 


\subsubsection{Population Dipole}

Population Dipoles were created by convolving the activity specific DRFs (EPSP, IPSP, spike related dipoles, etc.) with the thalamocortical network activity. Voltage traces of each neuron from the thalamocortical simulations were reduced to spike trains through a two step process. At each time point the voltage trace was checked to see if the membrane voltage exceeded a threshold voltage, indicating the occurrence of a spike. The threshold voltage was unique for each neuron type based on the specific resting and peak voltages, and set to $-50 \mathrm{mV},-40 \mathrm{mV},-50 \mathrm{mV}$, and $-60 \mathrm{mV}$ for the PY, IN, TC, and RE neurons respectively. When the voltage exceeded threshold, the preceding time point was checked to determine if it was below the threshold voltage to limit event detection to the rising edge of the spike. If both conditions were true a 1 was entered at that time point in the spike train array. For all other time points a zero was entered. The population dipole was formed subsequently by convolving each DRF generated in Section 3 with the binary spike train for the neuron which generated it. For the spiking contributions spike trains for the PY cells were convolved with the spiking DRF for a chattering PY cell, and spike trains for the IN cells were convolved with the spiking DRF for a spiny stellate cell.

Next the synaptic contributions were calculated. However, since each DRFs for the PSPs used a generic weight for their generation, they needed to be scaled to represent their actual impact on the population dipole. Scaling for the PSPs were done by normalizing the generic PSP from the NEURON model, to its related synapse in the thalamocortical network in order to account for the differences in conductance. Since both the synapses were conductance based and current scales linearly with conductance, we assume that the current dipole moment (which scales linearly with current as seen in equation 3.2) scales 
linearly with conductance. Thus a generic DRF for a PSP can be converted to a DRF for a specific synapse via by scaling the DRF by the ratio of the synaptic conductances as seen in the following equation:

$$
D R F_{\text {specific }}=D R F_{\text {generic }} \frac{G_{\text {specific }}}{G_{\text {generic }}}
$$

where $D R F_{\text {specific }}$ is the $\mathrm{DRF}$ for the specific synapse, $D R F_{\text {generic }}$ is the generic DRF for a PSP, $G_{\text {specific }}$ is the conductance of the specific synapse, and $G_{\text {generic }}$ is the conductance of the generic synapse. The specific DRF can then be convolved with the spike train for the presynaptic neuron generating the specific DRF (i.e. the neuron whose action potential will generate a postsynaptic potential on the postsynaptic neuron). The resulting dipole is then multiplied by the number of neurons the presynaptic is connected to. For example if a neuron is synaptically connected to 10 other neurons a single action potential by that neuron will generate 10 PSPs (one on each neuron it is connected to).

For PY-PY connections the generic DRF for an EPSP on a pyramidal cell was scaled according to the method above and using the AMPA mediated conductance listed in table 4.1 as $G_{\text {specific }}$ in equation 4.1.2. It was then convolved with the spike trains from PY cells and multiplied by 10 to account for the fact that each PY cell synapses onto its five nearest neighbours on either side. Similarly for PY-IN connections the generic DRF for an EPSP for a spiny stellate cell was scaled the AMPA mediated conductance listed in table 4.1 as $G_{\text {specific }}$. It was then convolved with the spike trains from PY cells and multiplied by 2 to account for the fact that each PY cell synapses onto the nearest IN cell on either side. For IN-PY connections the generic DRF for an IPSP on a pyramidal cell was scaled the $G A B A_{A}$ mediated conductance listed in table 4.1 as $G_{\text {specific }}$. It was then convolved with the spike trains from IN cells and multiplied by 10 to 
account for the fact that each IN cell synapses onto the five nearest PY cells on either side. Finally, synaptic contributions from TC-PY connections were calculated using the same methodology, but substituting the AMPA mediated conductance in table 4.1 as $G_{\text {specific }}$, the spike trains from TC cells and scaling by a factor of 20 to account for the number of PY cells each TC cell synapses with.

\subsection{Simulation of EEG Signal}

\subsubsection{Patient Data}

To verify the accuracy of the network simulations, the simulated population dipole was compared to recorded data of the same type. Electroencephalographic data was obtained through PhysioNet (Goldberger et al., 2000; Kemp et al., 2000), and downloaded from https://www.physionet.org/physiobank/database/sleep-edf/. The database contained eight polysomnograms (PSGs) from two separate studies (Kemp et al., 2000; Mourtazaev et al., 1995). PSGs are a multi-parametric data sets measured during sleep studies in which EEG, electrocardiography (ECG), electrooculography (EOG), and electromyography (EMG) data are collected simultaneously. Polysomnograms also include a hypnogram, which records the sleep phase data, used to isolate the portions of the data corresponding to slow-wave sleep.

For comparison with the simulated EEG activity, polysomnogram data from subject sc4002e0 (a healthy 33 year old female), was used. The data included 24 hours of continuous EEG recordings from a pair of differential electrodes ( $\mathrm{FpzCz}$ and $\mathrm{PzOz}$ ). EEG recordings were sampled at $1000 \mathrm{~Hz}$ and band pass filtered from $0.5 \mathrm{~Hz}$ to $100 \mathrm{~Hz}$. Recordings were then loaded into the polysomnogram 
reader Polyman (https://physionet.org/pn4/sleep-edfx/Polyman/), and sections of time where the hypnogram denoted slow wave seep (SWS) activity were marked for comparison with the simulated network activity. The data was then read into Matlab using the edfread function (https://www.mathworks.com/matlabcentral/fileexchange/31900-edfread). A 30 second portion of data was epoched out from one of the SWS time periods noted above for analysis.

\subsubsection{Forward Projection of Simulated Activity}

In order to compare the simulated population dipole with the patient EEG data the population dipole were forward projected to the scalp to estimate the corresponding EEG signals. Forward projection of the population dipole was performed with Brainstorm (Tadel et al., 2011), which is documented and freely available for download online under the GNU general public license (http://neuroimage.usc.edu/brainstorm). Since no patient specific anatomy was available, a boundary element model (BEM) of the standard conlin27 brain was created using Brainstorm's OpenMEEG BEM toolbox Gramfort et al., 2010; Kybic et al. 2005). Since the thalamocortical network chosen was specifically designed from a somatosensory network, population dipoles from SWS simulations were placed on a scout in the right somatosensory cortex. Pink noise (noise with power spectra $=1 / \mathrm{f}$ ) was used to simulate background physiologic activity in the brain (Musha and Yamamoto, 1997; Zhou et al., 2012). To simulate this, scouts, of identical size to the scout containing the population dipole, were added to every lobe in the brain, besides the right parietal lobe. These scouts were then used to add a pink noise signal which was power matched to the population dipole. The scouts were then used in a forward projection to 
obtain simulated EEG signals. The simulated EEG signals were then resampled at $1000 \mathrm{~Hz}$ and band pass filtered from $0.5 \mathrm{~Hz}$ to $100 \mathrm{~Hz}$ in order to match the sampling and filtering characteristics of the polysomnogram data. Finally, the simulated signal from electrode $\mathrm{Cz}$ was subtracted from $\mathrm{FPz}$ to create a simulated $\mathrm{FPzCz}$ signal. Additionally, electrode $\mathrm{Oz}$ was subtracted from $\mathrm{Pz}$ to create a simulated $\mathrm{PzOz}$ signal to compare simulated signals to recorded signals.

\subsection{Comparison of Simulations to Physiologic Data}

\subsubsection{Network Activity and the Population Dipole}

The thalamocortical network detailed by Bazenhov et al. (2002) and adapted here, was based on electrophysiological results reported by Contreas and Steriade (1995), which provides a point of comparison for the results of the population DRF. Fig. 4.2 shows example voltage traces corresponding to the membrane voltage from a sample pyramidal cell in the thalamocortical simulation (top) and the intracellular recording of a pyramidal cell from a cat in chemically induced slow wave sleep taken from Contreas and Steriade (1995) (bottom). Both sets of data show periods of vigorous bursting (up states) followed by periods of rest or inhibition (down states). The data reported in Contreas and Steriade (1995) transitioned between the up and down states at approximately $0.7 \mathrm{~Hz}$. Contreas and Steriade reportsed that this value falls within the range of previously reported values of $0.6 \mathrm{~Hz}$ to $0.9 \mathrm{~Hz}$. The simulated pyramidal neuron activity transitioned between the up and down states at approximately $0.6 \mathrm{~Hz}$ which is also within the reported range. Additionally, the two sets of data have similar duty cycles $(t(14)=0.8221, p=0.42)$ with the simulations having an average duty cycle of $0.59 \pm 0.13$ and the experimental 

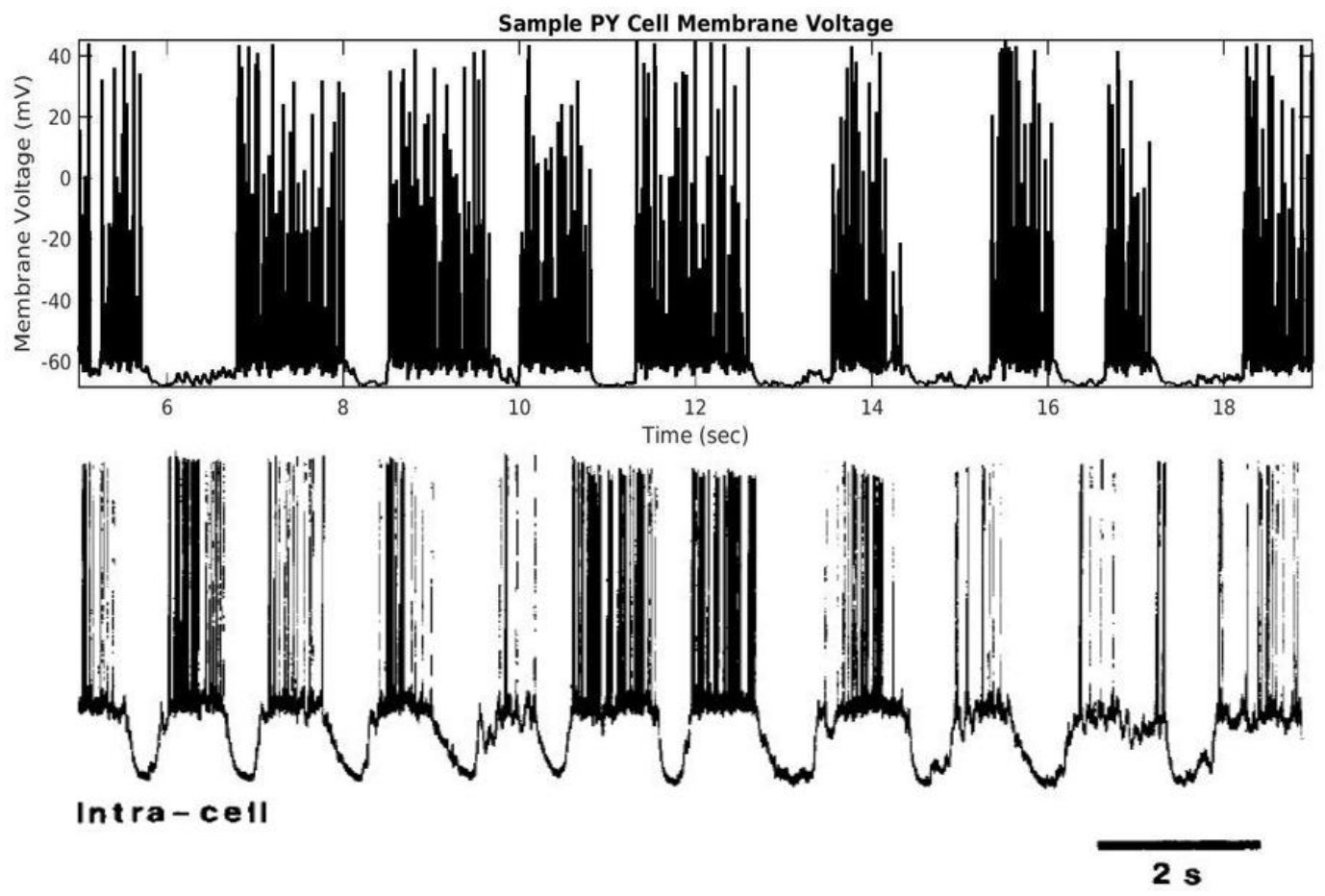

Figure 4.2: Comparison between simulated and recorded intracellular membrane voltage traces from sample pyramidal cells. (Top) Activity from a simulated pyramidal cell in a thalamocortical network during slow wave sleep. (Bottom) Activity from a pyramidal cell from a cat in chemically induced slow wave sleep; adapted from (Contreas and Steriade, 1995)

data having an average duty cycle of $0.55 \pm 0.12$.

Figure 4.3 shows a comparison of the simulated population dipole and the depth EEG obtained experimentally by Contreas and Steriade (1995). The depth EEG encapsulates what is currently considered the local field potential (LFP) and multi-unit activity (MUA) signals. Since the original experimental data could not be obtained, quantitative statistical comparisons could not be made. Qualitative comparisons showed that the simulated data had a lower fundamental frequency $(0.66 \mathrm{~Hz}$ vs $0.74 \mathrm{~Hz})$, however, both still fell within the reported range of $0.6 \mathrm{~Hz}$ to $0.9 \mathrm{~Hz}$ (Steriade et al., 1993). The data sets also had some additional features in common. First, both the signals had sharp negative peaks at the onset or slightly after $(<0.2 s)$ of pyramidal cell activity. Second, 

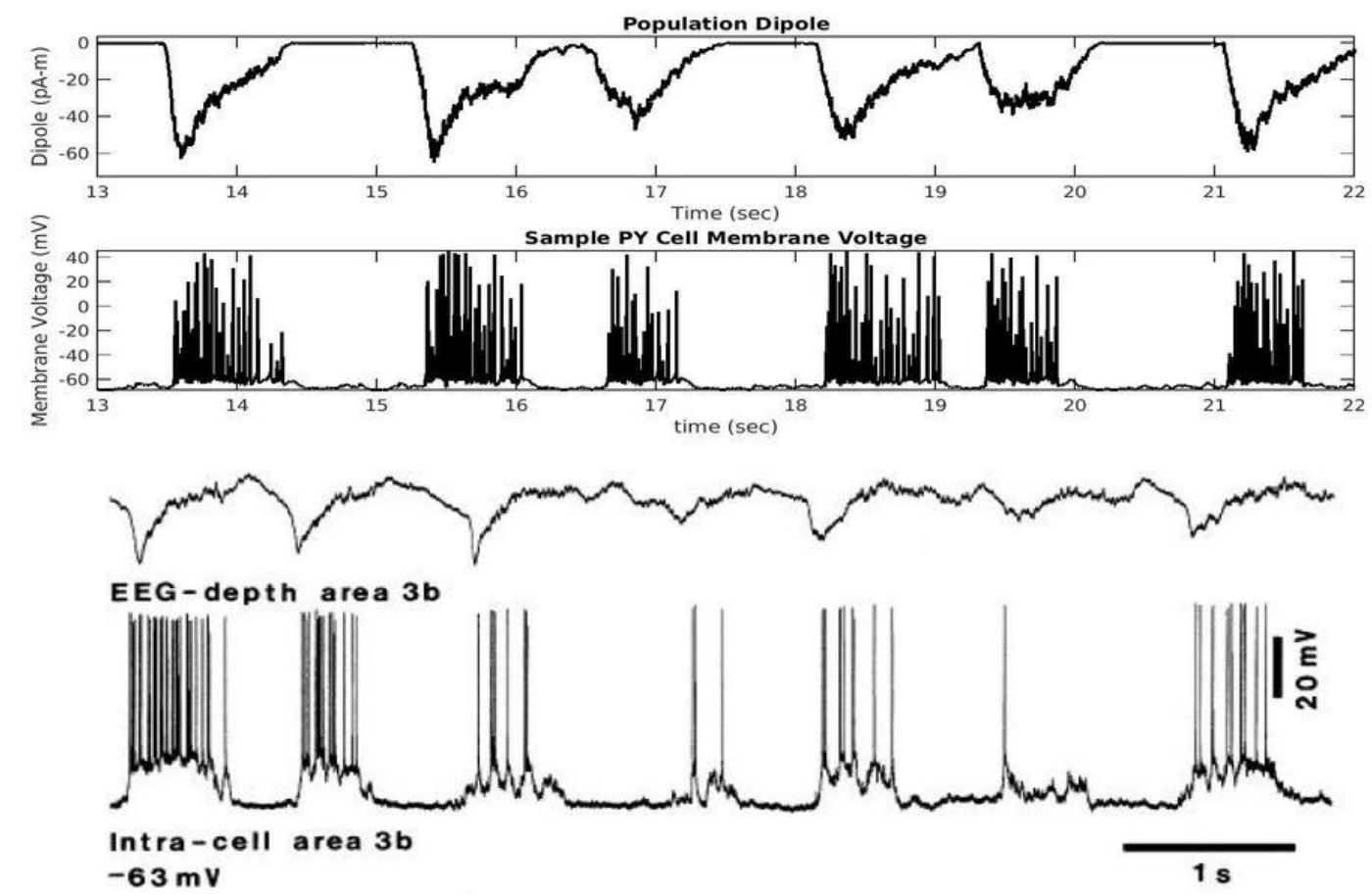

Figure 4.3: Comparison between simulated and recorded intracellular membrane voltage traces from pyramidal cells as well as population dipoles and depth EEGs. (A-Top) Population dipole for the thalamocortical network simulation shown together with the membrane voltage (A-Bottom) from the sample pyramidal cell shown in fig 4.2. (B-top) Depth EEG recorded from the somatosensory cortex of a cat in chemically induced slow wave sleep together with the intracellular recordings of a simultaneously measured pyramidal cell (B-Bottom); adapted from (Contreas and Steriade, 1995)

the relative sharpness of each negative peak appears to be related to the strength and duration of the pyramidal spiking activity, with large peaks occurring with large bursts of pyramidal cell activity and small almost non-existent peaks occurring where there is very little pyramidal cell activity.

\subsubsection{EEG Activity}

EEG activity associated with the simulated population was estimated by forward projection of the population current dipole onto the colin27 brain and 

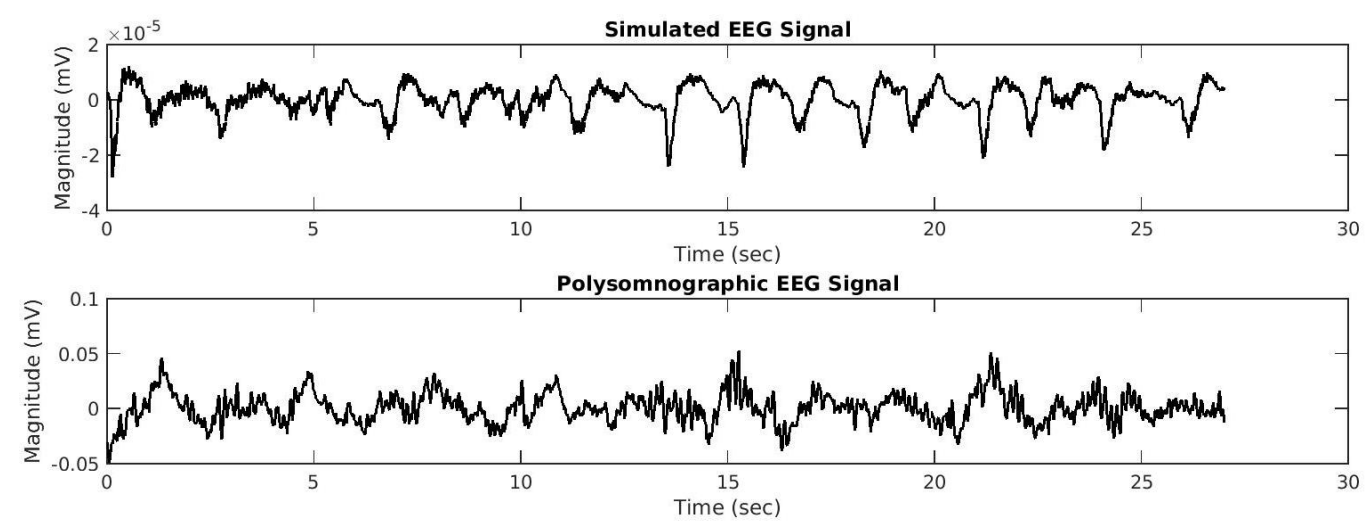

Figure 4.4: Comparison between simulated and recorded surface EEG signals for individuals in slow wave sleep. (Top) Simulated EEG signal. Signal was obtained by forward projecting the population dipole and then subtracting the Oz electrode data from $\mathrm{Pz}$ to obtain a signal similar to the polysomnographic data. (Bottom) A 25 second snippet of EEG signals from the polysomnogram obtained from physionet. Patient was a 33 year old healthy female and data shown is the $\mathrm{PzOz}$ signal.

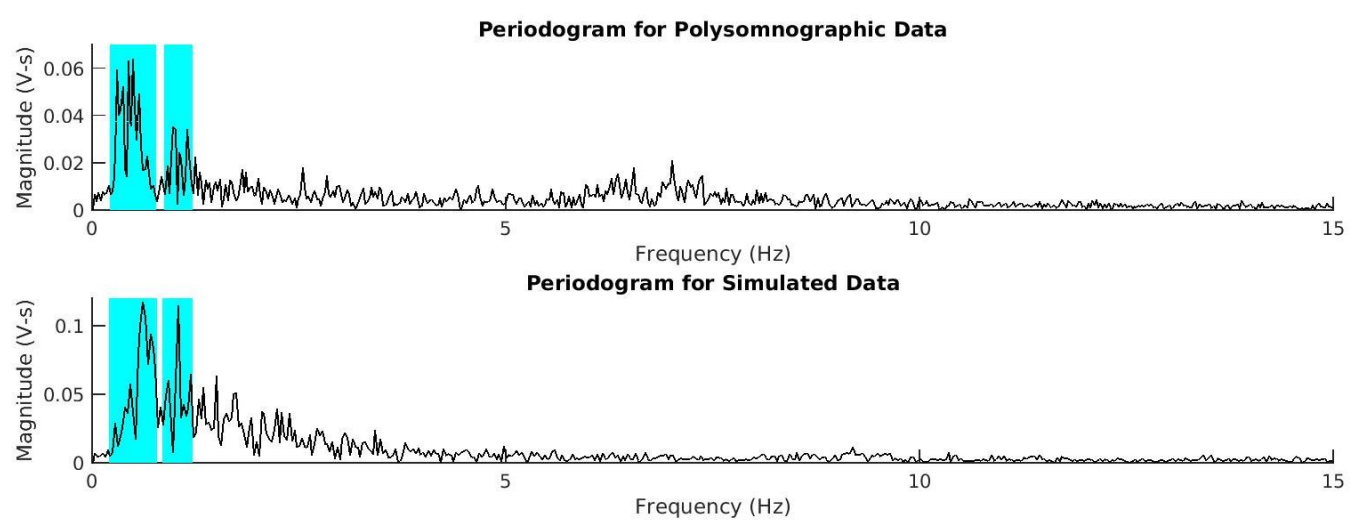

Figure 4.5: Comparison between simulated and recorded periodograms for individuals in slow wave sleep. (Top) Periodogram for the simulated EEG signals. (Bottom) Periodogram for the 25 second segment of surface EEG measured from a human subject; shown in fig. 4.4 
compared to recorded EEG signals from a 33 year old healthy female during slow wave sleep. Figure 4.4 shows example time series of both the simulated and experimental EEG data. Comparisons between the frequency spectra (figure 4.5) show that $\sim 25 \%$ of the power (27\% for simulated and $23 \%$ for subject data) occurred under $1 \mathrm{~Hz}$, with peaks at $0.6104 \mathrm{~Hz}$ and $0.4883 \mathrm{~Hz}$ for simulated and recorded signals respectively. Additionally, two bands in the frequency power can be seen in the recorded signal. A larger band from $0.22 \mathrm{~Hz}$ to $0.78 \mathrm{~Hz}$ and a smaller band from $0.87 \mathrm{~Hz}$ to $1.22 \mathrm{~Hz}$. Similar bands in the power of the simulated population dipole were observed with the lower frequency band ranging from $0.21 \mathrm{~Hz}$ to $0.79 \mathrm{~Hz}$, and the secondary band from $0.85 \mathrm{~Hz}$ to $1.22 \mathrm{~Hz}$.

\subsection{Discussion}

This section presents a novel technique for simulating EEG signals by convolving simulated network activity with the characteristic current dipoles generated by morphologically complex neurons (DRFs) then forward projecting the resulting population dipole onto the scalp. This technique was then validated at multiple spatial scales to ensure that the final EEG signals generated were reflective of underlying network spiking. Comparison of the simulated and recorded signals of a thalamocortical network at the neuronal level showed that the spiking of pyramidal neurons in the point source network had a spiking profile similar to the spiking profile of pyramidal neurons recorded in a cat. Most notably that frequency at which they transitioned between up and down states (0.6 Hz vs $0.7 \mathrm{~Hz}$ for simulated and recorded respectively), as well as the duty cycle of that transition $(0.59 \pm 0.13$ vs $0.55 \pm 0.12)$ were both within previously reported norms, or not statistically different $(t(14)=0.8221, p=0.42)$. When the population dipoles were compared to depth EEG recordings our finding 
showed that the population dipole and depth EEG recordings shared a similar fundamental frequency $(0.66 \mathrm{~Hz}$ vs $0.74 \mathrm{~Hz}$ respectively). Our results also showed that the two signals modulate the sharpness of each downward peak relative to the activity of the pyramidal cells in a similar fashion (e.g. large peaks corresponded with large bursts of pyramidal cell activity and small almost non-existent peaks corresponded where there is very little pyramidal cell activity). Finally, the population dipole was forward projected onto the scalp to validate that the results from the neuronal and population level analyses translated to the surface EEG. Our results demonstrated that the frequency spectra of simulated and experimental EEG were similar. Most significantly the frequency bands at which the signals contain a significant portion of their power below $1 \mathrm{~Hz}$. Additionally the spectra shared two distinct bands of high power from $0.22 \mathrm{~Hz}$ to $0.78 \mathrm{~Hz}$ and from $0.87 \mathrm{~Hz}$ to $1.22 \mathrm{~Hz}$ in the experimental EEG spectra, and from $0.21 \mathrm{~Hz}$ to $0.79 \mathrm{~Hz}$ and from $0.85 \mathrm{~Hz}$ to $1.22 \mathrm{~Hz}$ in the simulated spectra.

These findings suggest that the technique for simulating EEG signals presented in this section can relate EEG signals detectable at the scalp to neuronal level spiking activity. This can provide an important tool for studying brain dynamics, by allowing researchers to potentially relate non-invasive measures of brain activity, namely EEG signals, to cellular generators of those signals. 


\section{Evoked Potentials}

\subsection{Thalmocortical Evoked Response}

In section 4, comparison of the simulated and recorded signals of a thalamocortical network in slow wave sleep showed a number of similarities between the two signals. In particular figure 4.3 shows that not only does the pyramidal cell activity of the two networks bare remarkable similarities, but population level dipoles also modulate the sharpness of each downward peak relative to the activity of the pyramidal cells. Figure 4.5 shows there are some fairly distinct similarities between the periodograms of the simulated and patient data. Most significantly the frequency bands at which the signals contain a significant portion of their power below $1 \mathrm{~Hz}$. This suggests that the techniques described in sections 3.1.1 and 4.1.2 can provide a reasonable representation of dipole signals generated by a thalamocortical network in slow wave sleep.

To facilitate quantitative analyses the thalamocortical network was modified to produce an evoked potential for comparison with experimental measures of evoked activity. This was done not only to show that the technique can model multiple types of signals, but estim data records data from all electrodes and thus could be more quantitatively compared to the simulated signals. With all electrodes a source localization could be done on the experimental data. Instead of adding noise to our signal and forward projecting it, we could use source localization only focus on signals generated from a specific region, making it easier to compare the two signals.

The thalmocortical slow wave sleep network was modified to model the neuronal dynamics associated with an evoked response in the active network 
state by removing the sodium leakage currents in TC and PY cells (making them more excitable), while simultaneously reducing the maximum conductance of PY-PY AMPA mediated synapses by 40\% (Bazenhov et al. 2002). In the SWS state, the network tended to oscillate at its own intrinsic frequency regardless of input. However, in the active state the network became highly responsive to outside input, primarily from the thalamus.

In order to generate a somatosensory evoked response, electrical stimulation of a peripheral sensory nerve was simulated by stimulating the middle half of the neurons in the TC population with an external spiketrain generating PSPs on AMPA receptors on the TC cells. Stimulation occurred for $50 \mathrm{~ms}$ at random intervals with a mean rate of $2 \mathrm{~Hz}$ (Misulis and Head, 2003). To replicate averaging done across trials in an experimental setting 35 simulations with a duration of 15 seconds each were conducted. Initial membrane voltages for all neurons were randomly assigned at the start of each simulation to ensure variability between simulations.

\subsubsection{Population Dipole}

Population dipoles for each of the 35 simulations were obtained from the network using the approach described in Section 4.1.2. The normalization of synaptic dipoles was adjusted to reflect the 40\% decrease in AMPA mediated PY-PY connections. The dipoles were then parsed into 1 second epochs with 0.5 seconds on either side of the stimulation event to match the epoching done on the experimental data. The epochs were then averaged and low pass filtered at $50 \mathrm{~Hz}$. 


\subsubsection{Patient Data}

De-identified somatosensory evoked response data was analysed from a single subject during electrical stimulation (e-stim) of the common peroneal nerve where it crosses the head of the fibula. Stimulation was applied using a D67A, Digitimer Ltd. electrical stimulator. The stimulator used a bar electrode with two $1 \mathrm{~cm}$ diameter contacts with $2.5 \mathrm{~cm}$ between the electrodes. The nerve was stimulated at $90 \%$ motor threshold at $2 \mathrm{~Hz}$ for 2.5 seconds per trial. Motor threshold was identified by visual observation of twitch contraction prior to beginning of the trial. The specific purpose of the e-stim was to produce a robust evoked response in order to measure the cortical response to an ascending sensory signal. EEG data was obtained using a Brain Products 65 electrode actiCAP sampled at $2000 \mathrm{~Hz}$.

\subsubsection{Source Localization}

EEG data was preprocessed using the EEGLAB toolbox in MatLab (Delorme and Makeig, 2004). EEG recordings were first re-referenced to the average potential at each timepoint with the inclusion of the original reference

electrode FCz. Signals were then low-pass filtered with a fourth order Butterworth filter at $100 \mathrm{~Hz}$ and parsed into 1 second epochs with 0.5 seconds on either side of the stimulation event. Epochs were then passed into the AMICA algorithm (https://sccn.ucsd.edu/ jason/amica_web.html) for independent component analysis (ICA) and the resulting components were passed to the ADJUST algorithm for automatic rejection of artifact related components. Epochs were then averaged and the electrode data was passed into Brainstorm for source localization. A BEM was created for the colin27 brain using the 
OpenMEEG BEM toolbox. Brainstorm's wMNE algorithm was used to perform the source localization. The somatosensory cortex was labelled as a region of interest (ROI) and the mean activity from the vertices within the ROI were used to estimate the time course of the somatosensory dipole activity.

\subsection{Analysis}

\subsubsection{Bootstrap Analysis}

The relationship between the source localized EP and the simulated population dipole EP was characterized quantitatively using a bootstrap analysis of phase randomized EP timeseries. The correlation between the simulated and experimental timecourses was calculated between $\pm 150 \mathrm{~ms}$ of stimulus onset was calculated. Then the FFT of the source localized signal was taken to obtain the magnitude and phase spectra. The phase data was then randomized using Matlab's randperm function and the omplex FFT vector was reconstructed from the magnitude and randomized phase. The inverse FFT was then calculated using the Matlab ifft function to obtain a randomized time series with match magnitude spectra. The correlation between the phase randomized signal and the simulated population dipole was calculated, and the procedure was repeated 10,000 times to obtain a probability density function (PDF) of correlations. This allowed us to quantify the likelihood that the simulated population dipole would correlate with a random signal of identical power as a recorded EP.

\subsubsection{Correlation of Decomposed Simulated Signal}

The dipole contributions to the simulated signals for both the SWS and EP simulations were broken down into three components, pyramidal spiking, 
interneuron spiking, and post synaptic potentials. The pyramidal spiking signal corresponded to the portion of the population dipole obtained by convolving PY cell activity with the spike related DRF for a regularly spiking PY cell. The interneuron spiking signal reflected the contributions of the spike related DRF for an IN cell, and finally the post synaptic potential signal corresponded to the portion of the population dipole obtained by from all PSPs, both excitatory and inhibitory, on both PY and IN cells. Each of these components were subsequently correlated with the total population dipole to determine which components accounted for the most variance in the simulated signals.

\subsection{Evoked Potential}

Figure 5.1 shows a comparison between the simulated population dipole and the source localized subject data. The correlation between the the two signals was $0.43(\mathrm{p}=0.0036)$.

The simulated current dipole was decomposed into its constitutive elements (pyramidal spiking, interneuron spiking, and post synaptic potentials) to determine which elements contributed the most to the total dipole. Figure 5.2 shows the decomposed population dipole. The correlations of each sub-population dipole to the total population dipole 0.9884 for spike related dipoles from regularly spiking pyramidal cells, 0.1090 for spike related dipoles from inhibitory interneurons, and -0.1001 for dipoles resulting from synaptic activity on both pyramidal cells and interneurons. 
Source Localization vs Simulation

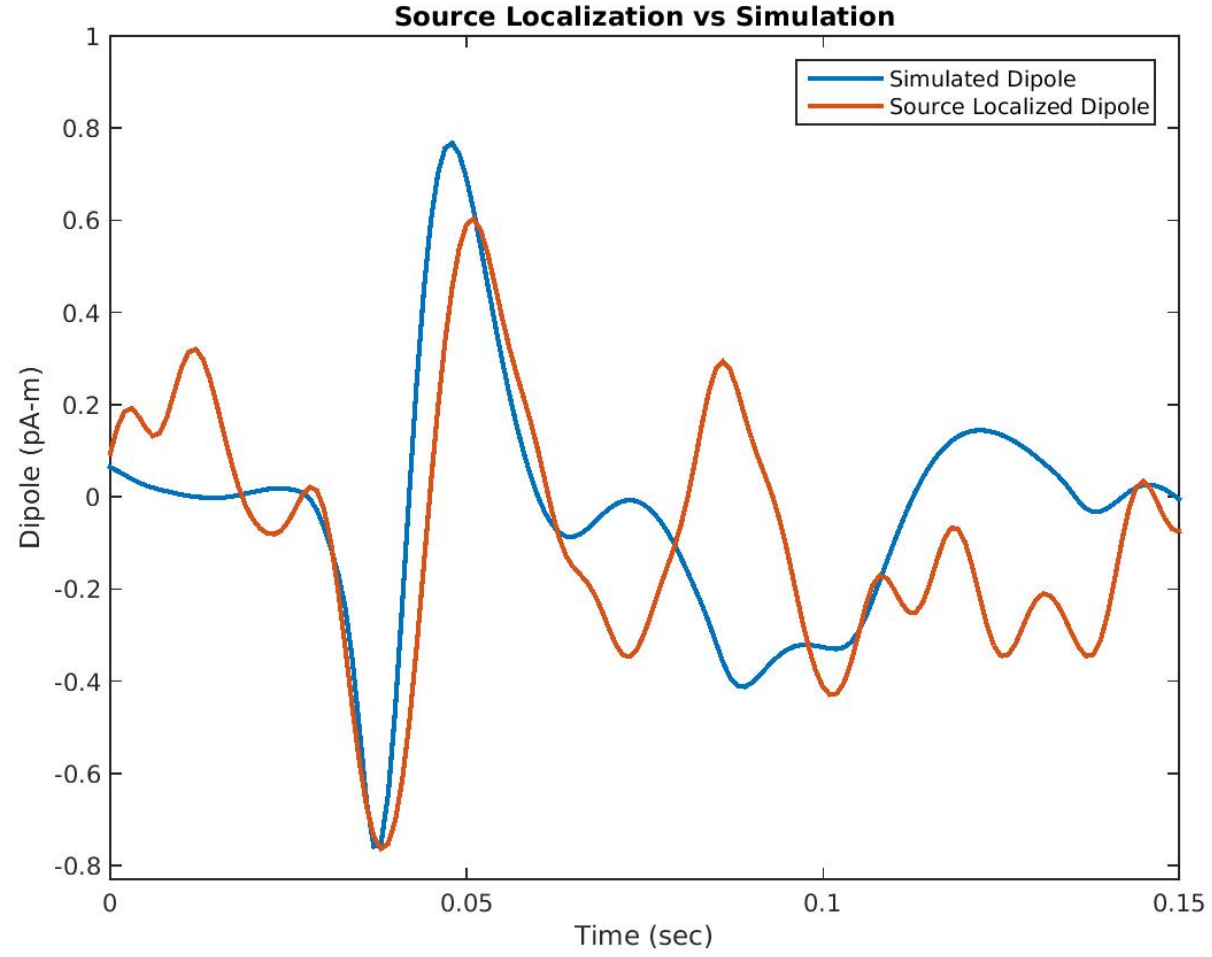

Figure 5.1: Time course of the current dipole sources for an evoked potential. Orange trace shows the source localized signal obtained from recorded EEG data. Blue trace shows the simulated population dipole. 


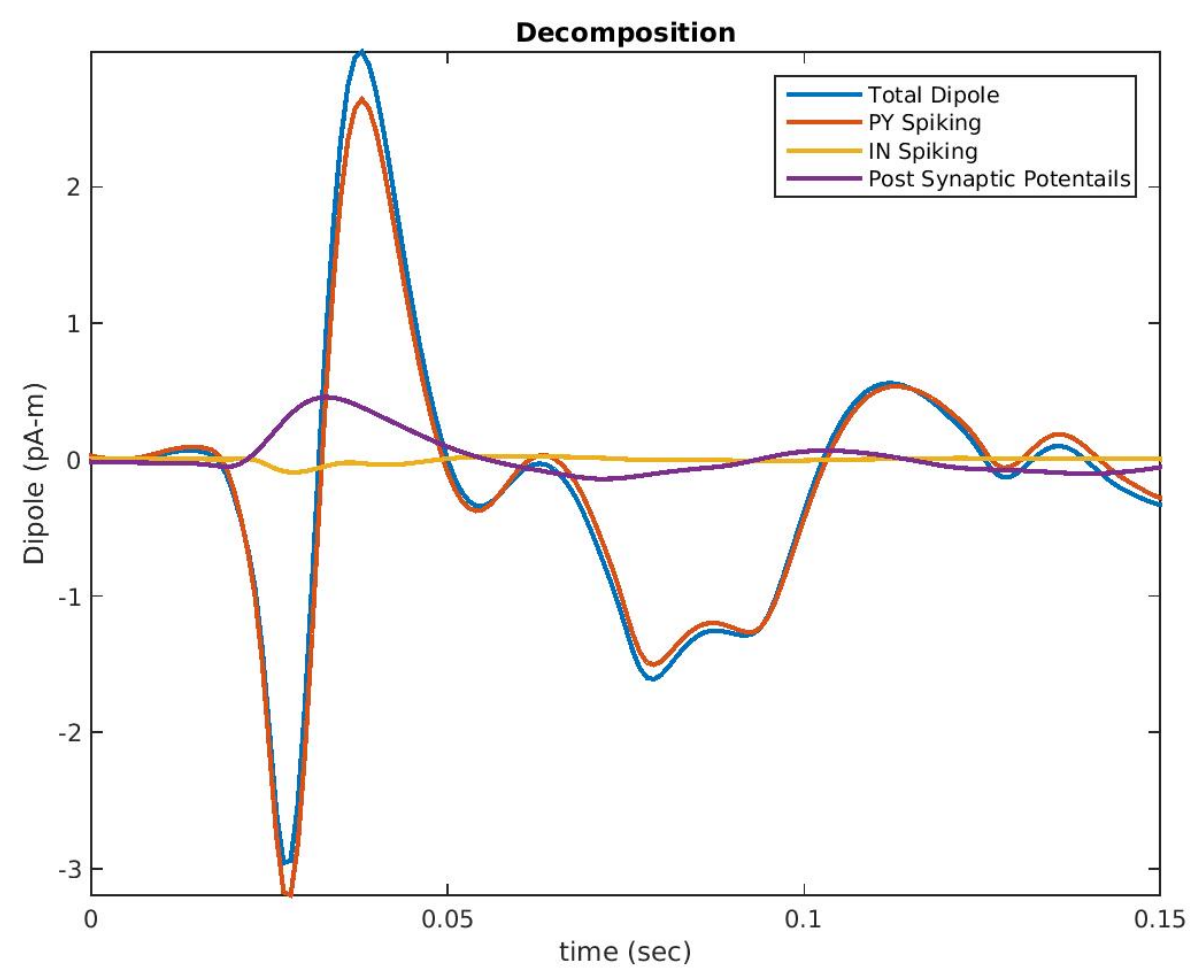

Figure 5.2: Total population dipole (blue) superimposed on the individual components, PY spiking (orange), IN spiking (yellow), and PSPs (purple) relative to stimulus onset. 


\subsection{Discussion}

Evoked potentials were chosen because they are a physiologically realistic, clinically relevant, easily simulated waveform that could be incorporated into the thalamocortical network model. The results make several interesting points. The first, to provide a quantitative look at how well the method of constructing DRFs and convolving them with network activity may be used to represent signals in the brain. The correlation between the simulated and measured dipole activity was $\mathrm{r}=0.43(\mathrm{p}=0.0036)$. The second, best visualized in figure 5.2 . shows the relative contributions of each type of cellular event (PY spike related dipoles, IN spike related dipoles, and PSPs) to the total signal. Examination of the magnitude of the signals reveals that the pyramidal cell spike related dipoles are over twice as large as the contributions from all other synaptic sources, accounting for $98.8 \%$ of the variability in the total population dipole.

Due to the challenges of obtaining intercellular recordings simultaneously with EEG in hman subjects, few studies have examined the cellular contributions to EEG. Murakami et al. (2002) examined the relative contributions of directly stimulated (spiking) pyramidal cells versus those that are synaptically stimulated as a result of spiking activity. They found that the directly stimulated neurons had an almost equal contribution to the current dipole as neurons stimulated via synaptic activity.

Finally, the evoked potential is an extremely fast waveform, consisting of a P37 and N45, followed by another positivity at around $60 \mathrm{~ms}$ and a final negativity at approximately $75 \mathrm{~ms}$. Each peak is approximately 10 to $15 \mathrm{~ms}$ apart. With post synaptic potentials being 20 to $30 \mathrm{~ms}$ long it would be difficult for them to sum in manner which could create a waveform with a higher 
frequency content. One such way would be if pyramidal cells were located on opposite sides of a sulcal wall. The resulting PSPs would then have opposite signs. However, this would require a very specific orientation of cells which fired with a timing precise enough to cause destructive interference. Despite the fact that evoked potentials could be due to either spiking activity or opposing PSPs, the more parsimonious reason would be the first one, especially considering that the sulcal walls on the cortex produce dipoles parallel to the scalp. 


\section{Discussion}

The most common assumption made with regard to the physiological origins of EEG signals is that they arise from post synaptic potentials on synchronously firing pyramidal cells in the cortex of the brain. However, recent studies and literature reviews suggest that the physiological origins of EEG may not as certain as once thought (Cohen, 2017; Riera et al., 2012; Buzsàki et al., 2012; Anastassiou et al., 2015; Herreras, 2016; Reimann et al., 2013). In particular, Reimann et al. (2013) assert that spiking activity is detectable in LFPs for frequencies low as $50 \mathrm{~Hz}$ while Anastassiou et al. (2015) indicate that action potentials could significantly impact the LFP at a frequency as low as 20 Hz. Although there is not a one-to-one correlation between LFPs and EEG signals, they are both considered reflect the sum of extracellular currents generated primarily by synaptic activity (Lopes da Silva, 2010, Olejniczak, 2006, Herreras, 2016; Hagen et al., 2016). Thus uncertainty in the origin of LFPs also raises questions about the about the origin of EEG signals.

This is reinforced by the results in Section 3.2 which showed that dendritic calcium currents activated by backpropigation of currents due to an action potential generate dipoles which have a magnitude and duration sufficient to generate detectable EEG signals. Findings which are consistent with the literature (Stuart et al., 2008; Suzuki and Larkum, 2017). Additionally, a study by Buzsàki et al. (1988) showed that, in rats, afterhyperpolarizations in layer V pyramidal cells can contribute to the extracellular field, and that they are the most likely source of delta waves recorded during their experimentation. This is consistent with our own findings in Section 3.2 which showed that afterhyperpolarizations are reflected in the current dipole moment generated by a regularly spiking pyramidal cell and have a duration (18 ms) which could lend 
itself to temporal summation.

Aside from questions about the current understanding of the cellular origins of EEG, other concerns have been raised about the current state of EEG research. A review by Cohen (2017) asserted that while the standard model of EEG (defined as the 'integration of postsynaptic potentials across neural populations') explains the existence of EEG signals, it does nothing to elucidate its content or meaning. The simulation results reported in Sections 4.3 and 5.3 take a first step toward linking activity of individual neurons to EEG data thus elucidating the content of the EEG signal. Cohen goes on to state that because the standard model is primarily used as a justification that EEG signals exist instead of as a tool for analysing EEG, the current state of the art EEG analysis tend to be macroscopically focused. They rely on correlation analyses without being able to determine whether the signals are epiphenomenal or actually reflective of underlying computations. Cohen asserts that best way to address the issue of determining whether EEG signals are epiphenomenal or reflective of neural computations is to engage in research which focuses more on the recording and analyses of multiscale datasets (simultaneous recordings of individual neurons, LFPs, and EEG data). The research presented here addresses this by simulating a multiscale dataset which can be used to explore how underlying network activity reflects EEG activity. Specifically the results in Sections 4 and 5 suggest that EEG signals are not epiphenomenal, and can be reasonably predicted from the underlying network activity when combined with detailed knowledge of the electrophysiology and morphology of the neurons within the network.

Cohen also stressed the importance of understanding the microcircuit architecture is important to understanding specific neural processes like 
cognition. He noted that though source localization can be important for fields like retinotopic mapping and localizing ictal regions for surgical intervention. However, in fields like cognition, the microcircuit responsible for computation is more important. The simulation results provide important insights toward understanding microcircuitry contributions to EEG signals. As Cohen notes the literature surrounding microcircuitry has been steadily growing over the years Cohen, 2017; Gordon Shepherd and Sten Grillner, 2010). With the large numbers of anatomically detailed neurons in the ModelDB database (McDougal et al. 2017) it is possible to combine the techniques developed here with anatomically detailed NEURON models and microcircuitry literature within brain regions (Gordon Shepherd and Sten Grillner, 2010) to investigate which microarchitectures generate dipoles that best match source localized EEG signals. This type of analysis would give greater insight into how the brain processes information, and how EEG measures relate to the underlying microcircuits.

The point of this thesis is twofold. The first is the most obvious; to examine how individual neurons generate DRFs, and how those DRFs contribute to population dipoles which can be detectable by EEG electrodes on the scalp. More specifically, to computationally investigate the assumption that it is post synaptic potentials on synchronously firing pyramidal cells of the cortex that are the primary progenitors of elecroencephalographic signals. However, there is a secondary, less explored, motive to this thesis. That is to provide researchers with a way to break down EEG signals in a way which elucidates specifics about the network, and network activity, which gives rise to specific EEG signals. 


\section{Conclusion}

In 1947 Lorente de No published his seminal paper on EEG theory. The mathematics heavily involved an analysis of how current from action potentials travelling down axons disseminate through neural tissue. Seventy years later, action potentials are considered to not be involved in contributing to EEG signals at all, and it is, ironically, Lopes da Silva's work which is cited when making this assertion. Using computational methods we investigated the common assumption that EEG signals originate from post synaptic potentials on synchronously firing pyramidal cells on the cortex of the brain by examining how different cells and different cellular events contribute to population level dipoles. We then validated our results by comparing our population dipoles to recorded depth EEG signals as well as source localized EEG data. We showed that characterizing the morphological and electrophysiological properties of a neuron in terms of a dipole response function, and convolving the dipole response function with the corresponding neural network provided an accurate representation of biologically recorded signals. Additionally, this thesis examined the factors that most influenced the population dipole. Using a simulation of a somatosensory evoked potential, we show that the spike related dipoles from regularly spiking pyramidal cells were the primary driver of the signal. It is our hope that this technique will lead to a deeper understanding of how EEG signals are generated, and more importantly of the types of network dynamics that underlay specific EEG signals. 


\subsection{Future Directions}

Future efforts to expand on this research should be focused on three areas. First, expanding the size of the neural networks. Preliminary pilot data suggests that the weighting of post synaptic potentials in the thalamocortical network is not inversely proportional to network size. That is, if the network size doubles, the weighting of individual synapses does not reduce by half. Since, the population dipoles for the PSPs are normalized by the weights in the network, this means that the ratio of spiking DRFs to PSP DRFs at the population level will change. However, to what extent this affects the population dipole, especially for much larger networks, is unknown.

The second focus should be expanding the approach by adding DRFs from additional neuron models. Additionally, it has been suggested by Riera et al. (2012) that monopoles may also play a role in generating EEG signals. Though the paper suggests that monopoles are involved, they do not postulate a method by which neurons generate monopoles. It could be worthwhile to investigate how neurons might generate monopoles and if these monopoles could be modelled and included in the method (i.e. adding a monopole response function to the model).

Finally, the DRF approach could be applied to other networks. Evoked potentials and slow wave sleep networks were used here as a proof of concept, however, a wide variety of networks have been studied using EEG. As stated in section 3, an objective of the research was to allow for a more in-depth analysis of EEG signals. More specifically, to use EEG signals to elucidate the dynamics inside brain networks or microcircuits of interest for a given research task. This is possibly the most interesting and useful of the three possible future directions. While understanding the origins of EEG activity is important, it is only useful 
insofar as it informs us of the functions of the human brain. 


\section{A Appendix: Cellular Currents}

\section{A.1 NEURON Models}

For both the pyramidal cell and the spiny stellate cell, the dendrites contain the sodium current, slowly activating potassium current, calcium dependent potassium current, and calcium current. The soma includes all of the above currents in addition to a fast activating potassium current. The axonal hillock contains only sodium and fast activating potassium currents. Myelin sheath and node segments contain only sodium currents. In addition to the active currents listed, all compartments have both capacitive and passive currents. For the regularly spiking pyramidal cell the calcium and calcium dependant potassium currents are removed.

Note that most of these equations were derived experimentally at room temperature $\left(23 C^{\circ}\right)$. However, the body is much warmer $\left(37 C^{\circ}\right)$. Therefore the rates must be adjusted. This is done through the variable $t_{a d j}$. Finally, these equations are transcribed from the ${ }^{*}$.mod files found in the Mainen models used in this thesis (Mainen and Sejnowski, 1996).

\section{Sodium Current}

Constants:

$$
\begin{array}{ll}
\bar{g}_{K}=1000\left(\frac{p S}{\mu m}\right) & \text { temp }=23\left(C^{\circ}\right) \quad \mathrm{q} 10=2.3 \text { (unitless) } \\
\text { Celsius }=37\left(C^{\circ}\right) & t_{a d j}=q 10^{(\text {celsius-temp }) / 10}
\end{array}
$$

Governing Equations: 


$$
\begin{aligned}
i_{N a} & =0.0001 * g_{n a}\left(V_{m}-E_{N a}\right) & g_{N a} & =t_{a d j} * \bar{g}_{N a} m^{3} h \\
\frac{d m}{d t} & =\frac{m_{\infty}-m}{m_{\tau}} & m_{\tau} & =\frac{1}{t_{a d j}\left(\alpha_{m}+\beta_{m}\right)} \\
m_{\infty} & =\frac{\alpha_{m}}{\alpha_{m}+\beta_{m}} & \alpha_{m} & =1.638 * \operatorname{efun}\left(\frac{-35-V_{m}}{9}\right) \\
\beta_{m} & =1.116 * \operatorname{efun}\left(\frac{V_{m}+35}{9}\right) & \frac{d h}{d t} & =\frac{m_{\infty}-m}{m_{\tau}} \\
h_{\tau} & =\frac{1}{t_{a d j}\left(\alpha_{h}+\beta_{h}\right)} & h_{\infty} & =\frac{1}{1+\exp \left(\frac{V_{m}+65}{6.2}\right)} \\
\alpha_{h} & =0.12 * \operatorname{efun}\left(\frac{-\left(50+V_{m}\right)}{5}\right) & \beta_{h} & =0.0455 * \operatorname{efun}\left(\frac{-\left(75+V_{m}\right)}{5}\right)+1
\end{aligned}
$$

Where efun is a modified exponential function defined as follows:

$$
\operatorname{efun}(z)= \begin{cases}1-\frac{z}{2} & \text { ifabs }(z)<0.0001 \\ \frac{z}{\exp (z)-1} & \text { ifabs }(z)>0.0001\end{cases}
$$

\section{Fast Activating Potassium Current}

Constants:

$$
\begin{aligned}
& \bar{g}_{K v}=5\left(\frac{p S}{\mu m}\right) \quad \text { temp }=23\left(C^{\circ}\right) \quad \text { celsius }=37\left(C^{\circ}\right) \\
& \mathrm{q} 10=2.3 \text { (unitless) } \quad t_{a d j}=q 10^{(\text {celsius-temp) } / 10}
\end{aligned}
$$

Governing Equations:

$$
\begin{array}{rlrl}
i_{K v} & =0.0001 * g_{K v} *\left(V_{m}-E_{K}\right) & g_{K v} & =t_{a d j} * \bar{g}_{K v} * n \\
\frac{d n}{d t} & =\frac{n_{\infty}-n}{n_{\tau}} & n_{\tau} & =\frac{1}{t_{a d j}\left(\alpha_{n}+\beta_{n}\right)} \\
n_{\infty} & =\frac{\alpha_{n}}{\alpha_{n}+\beta_{n}} & \alpha_{n}=0.18 * \operatorname{efun}\left(\frac{-\left(V_{m}-25\right)}{9}\right) \\
\beta_{n} & =0.018 * \text { efun }\left(\frac{\left(V_{m}-25\right.}{9}\right) &
\end{array}
$$

Where efun is the same modified exponential function defined for the sodium current.

\section{Slow Activating Sodium Current}

Constants: 


$$
\begin{array}{ll}
\bar{g}_{K m}=10(\mathrm{pS} / \mathrm{um} 2) & \text { temp }=23\left(C^{\circ}\right) \\
\text { celsius }=37\left(C^{\circ}\right) & \mathrm{q} 10=2.3(\text { unitless }) \\
t_{\text {adj }}=q 10^{(\text {celsius-temp }) / 10} &
\end{array}
$$

Governing Equations:

$$
\begin{array}{ll}
i_{K m}=0.0001 * g_{K m} *\left(V_{m}-E_{K m}\right) & g_{K m}=t_{a d j} * \bar{g}_{K m} * n \\
\frac{d n}{d t}=\frac{n_{\infty}-n}{n_{\tau}} & n_{\tau}=\frac{1}{t_{a d j} *\left(\alpha_{n}+\beta_{n}\right)} \\
n_{\infty}=\frac{\alpha_{n}}{\alpha_{n}+\beta_{n}} & \alpha_{n}=0.009 * \operatorname{efun}\left(\frac{-\left(V_{m}+30\right)}{9}\right) \\
\beta_{n}=0.009 * \operatorname{efun}\left(\frac{V_{m}+30}{9}\right) &
\end{array}
$$

Where efun is the same modified exponential function defined for the sodium current.

\section{Calcium Dependant Potassium Current}

Constants:

$$
\begin{array}{ll}
\bar{g}_{K C a}=10(\mathrm{pS} / \mathrm{um} 2) & C a_{i x}=1 \text { (unitless) } \\
\text { temp }=23\left(C^{\circ}\right) & \text { celsius }=37\left(C^{\circ}\right) \\
\mathrm{q} 10=2.3 \text { (unitless) } & t_{a d j}=q 10^{(\text {celsius-temp }) / 10}
\end{array}
$$

Governing Equations:

$$
\begin{array}{ll}
i_{K C a}=0.0001 * g_{K C a} *\left(V_{m}-E_{K C a}\right) & g_{K C a}=t_{a d j} * \bar{g}_{K C a} * n \\
\frac{d n}{d t}=\frac{n_{\infty}-n}{n_{\tau}} & n_{\tau}=\frac{1}{t_{a d j}\left(\alpha_{n}+\beta_{n}\right)} \\
n_{\infty}=\frac{\alpha_{n}}{\left.\alpha_{n}+\beta_{n}\right)} & \alpha_{n}=0.001 *[C a]_{i n}^{C a_{i x}} \\
\beta_{n}=0.02 &
\end{array}
$$

Note that $[\mathrm{Ca}]_{\text {in }}$ is the concentration of calcium inside the cell. It is driven by the calcium current.

\section{Calcium Current}


Constants:

$$
\begin{array}{ll}
\bar{g}_{N a}=0.1(\mathrm{pS} / \mathrm{um} 2) & \text { temp }=23\left(C^{\circ}\right) \\
\mathrm{q} 10=2.3(\text { unitless }) & \text { celsius }=37\left(C^{\circ}\right) \\
t_{a d j}=q 10^{\frac{\text { celsius-temp }}{10}} & E_{C a}=140(\mathrm{mV})
\end{array}
$$

Governing Equations:

$$
\begin{aligned}
i_{C a} & =0.0001 * g_{C a} *\left(V_{m}-E_{C a}\right) & g_{C a} & =t_{a d j} * \bar{g}_{C a} m^{2} h \\
\frac{d m}{d t} & =\frac{m_{\infty}-m}{m_{\tau}} & m_{\tau} & =\frac{1}{t_{a d j}\left(\alpha_{m}+\beta_{m}\right)} \\
m_{\infty} & =\frac{\alpha_{m}}{\alpha_{m}+\beta_{m}} & \alpha_{m} & =0.209 * \operatorname{efun}\left(\frac{-\left(27+V_{m}\right)}{3.8}\right) \\
\beta_{m} & =0.94 * \exp \left(\frac{-\left(75+V_{m}\right)}{17}\right) & \frac{d h}{d t} & =\frac{m_{\infty}-m}{m_{\tau}} \\
h_{\tau} & =\frac{1}{t_{a d j}\left(\alpha_{h}+h_{\beta}\right)} & h_{\infty} & =\frac{\alpha_{h}}{\alpha_{h}+\beta_{h}} \\
\alpha_{h} & =0.000457 * \exp \left(\frac{-\left(13+V_{m}\right)}{50}\right) & \beta_{h} & =\frac{0.0065}{\exp \left(\frac{-\left(15+V_{m}\right)}{28}\right)+1}
\end{aligned}
$$

Where efun is the same modified exponential function defined for the sodium current.

\section{Calcium Concentration}

Constants:

$$
\begin{aligned}
& \text { depth }=.1(\mathrm{um}) \quad \tau_{r}=200(\mathrm{~ms}) \\
& C a_{\infty}=100 \mathrm{e}-6(\mathrm{mM})
\end{aligned}
$$

Governing Equations:

$$
\begin{gathered}
\frac{d[C a]_{i n}}{d t}=\text { drive }+\frac{C a_{\infty}-[C a]_{i n}}{\tau_{r}} \\
\text { drive }= \begin{cases}\frac{-10000 * i_{C a}}{2 * F * \text { depth }} & \text { ifdrive }>0 \\
0 & \text { ifdrive } \leq 0\end{cases}
\end{gathered}
$$

Where F is Faraday's Constant. 


\section{A.2 Point Source Models}

Note that most of these equations were derived experimentally at room

temperature $\left(24 C^{\circ}\right)$. However, the body is much warmer $\left(36 C^{\circ}\right)$. Therefore the rates must be adjusted. This is done through the variable denoted as $\Phi_{x}$ or $T_{a d j}$

Additionally, some of these currents are derived from a paper written by Dr. Robert Traub. He uses resting potential as $V_{m}=0$, however, the neuron models use the absolute difference between the inner and outer potentials. To correct for this the variable $V_{t r}$ is used. Also note that $V_{t r}$ is only used for equations relating to the gating variables and not the ionic current. This is because the ionic current is driven by the absolute difference between the inner and outer potentials and therefore needed to be adjusted for in the Traub models, but does not need to be adjusted here.

Finally, these equations have been transcribed from the neur271.c code used in thalamocortical network model used in this thesis (Bazenhov et al. 2002).

\section{Fast Sodium Current (Cortical Cells)}

Constants:

$$
\text { Cels }=36 \quad \Phi=2.3^{(\text {Cels-23) } / 10}
$$

Governing Equations: 


$$
\begin{array}{ll}
i_{N a}=g_{N a} *\left(V_{m}-E_{N a}\right) & g_{N a}=\Phi * \bar{g}_{N a} m^{3} * h \\
\frac{d m}{d t}=\frac{-\left(m-m_{\infty}\right)}{\tau_{m}} & \tau_{m}=\frac{1}{\left(\alpha_{m}+\beta_{m}\right) * \Phi} \\
m_{\infty}=\frac{\alpha_{m}}{\alpha_{m}+\beta_{m}} & \alpha_{m}=0.182 * \frac{V_{m}+35}{1-\exp \left(\frac{-\left(V_{m}+35\right.}{9}\right)} \\
\beta=0.124 * \frac{-\left(V_{m}+35\right.}{1-\exp \left(\frac{-(V m+35)}{9}\right)} & \frac{d h}{d t}=\frac{-\left(h-h_{\infty}\right)}{\tau_{h}} \\
\tau_{h}=\frac{1}{\left(\alpha_{h}+\beta_{h}\right) * \Phi} & h_{\infty}=\frac{1}{1+\exp \left(\frac{V_{m}+65}{6 .}\right)} \\
\alpha_{h}=0.024 * \frac{V_{m}+50}{1-\exp \left(\frac{-\left(V_{m}+50\right.}{5}\right)} & \beta_{h}=0.0091 * \frac{-75)}{1-\exp \left(\frac{V_{m}+75}{5}\right)}
\end{array}
$$

\section{Fast Potassium Current (Cortical Cells)}

Constants:

$$
\text { Cels }=36 \quad T_{a d j}=9^{(\text {Cels-23) } / 10}
$$

Governing Equations:

$$
\begin{aligned}
i_{K v} & =g_{K v} *\left(V_{m}-E_{K v}\right) & g_{K v} & =T_{a d j} * \bar{g}_{K v} * m \\
\frac{d m}{d t} & =\frac{-\left(m-m_{\infty}\right)}{\tau_{m}} & \tau_{m} & =\frac{1}{\left(\alpha_{m}+\beta_{m}\right) * T_{a d j}} \\
m_{\infty} & =\frac{\alpha_{m}}{\alpha_{m}+\beta_{m}} & \alpha_{m} & =0.02 * \frac{V_{m}-25}{1-\exp \left(\frac{-\left(V_{m}-25\right.}{9}\right)} \\
\beta_{m} & =-0.002 * \frac{V_{m}-25}{1-\exp \left(\frac{V_{m}-25}{9}\right)} & &
\end{aligned}
$$

\section{Persistent Sodium Current (Cortical Cells)}

Constants:

$$
\text { Cels }=36 \quad \Phi=2.7^{(\text {Cels-23)/10 }}
$$

Governing Equations:

$$
\begin{aligned}
i_{N a p} & =g_{N a p} *\left(V_{m}-E_{N a}\right) & g_{N a p}=\bar{g}_{N a p} * m \\
\frac{d m}{d t} & =\frac{-\left(m-m_{\infty}\right)}{\tau_{m}} & \tau_{m}=\frac{0.8}{\Phi} \\
m_{\infty} & =\frac{0.02}{1+\exp \left(\frac{-\left(V_{m}+42\right)}{5}\right)} &
\end{aligned}
$$




\section{Potassium M Current (Cortical Cells)}

Constants:

$$
\text { Cels }=36 \quad T_{a d j}=2.3^{(\text {Cels-23)/10 }}
$$

Governing Equations:

$$
\begin{array}{ll}
i_{K m}=T_{a d j} * \bar{g}_{K m} * m *\left(V_{m}-E_{K m}\right) & \frac{d m}{d t}=\frac{-\left(m-m_{\infty}\right)}{\tau_{m}} \\
\tau_{m}=\frac{1}{(\alpha+\beta) * T_{a d j}} & m_{\infty}=\frac{\alpha_{m}}{\alpha_{m}+\beta_{m}} \\
\alpha=0.001 * \frac{V_{m}+30}{1-\exp \left(\frac{-\left(V_{m}+30\right)}{9}\right)} & \beta=-0.001 * \frac{v+30}{1-\exp \left(\frac{v+30}{9}\right)}
\end{array}
$$

Ca Dependent Potassium Current (Cortical Cells)

Constants:

$$
\text { Cels }=36 \quad T_{a d j}=2.3^{(\text {Cels-23)/10 }}
$$

Governing Equations:

$$
\begin{array}{ll}
i_{K C a}=T_{a d j} * \bar{g}_{K C a} * m *\left(V_{m}-E_{K C a}\right) & \frac{d m}{d t}=\frac{-\left(m-m_{\infty}\right)}{\tau_{m}} \\
\tau_{m}=\frac{1}{\left(\alpha_{m}+\beta_{m}\right) * T_{a d j}} & m_{\infty}=\frac{\alpha_{m}}{\alpha_{m}+\beta_{m}} \\
\alpha_{m}=0.01 *[C a]_{i n} & \beta_{m}=0.02
\end{array}
$$

High-Threshold Ca Current (Cortical Cells)

Constants:

$$
\text { Cels }=36 \quad \Phi=2.3^{(\text {Cels-23) } / 10}
$$

Governing Equations: 


$$
\begin{array}{ll}
i_{H V A}=\Phi * \bar{g}_{H V A} * m^{2} * h *\left(V_{m}-E_{C a}\right) & \\
\frac{d m}{d t}=\frac{-\left(m-m_{\infty}\right)}{\tau_{m}} & \tau_{m}=\frac{1}{\left(\alpha_{m}+\beta_{m}\right) * \Phi} \\
m_{\infty}=\frac{\alpha_{m}}{\alpha_{m}+\beta_{m}} & \alpha_{m}=0.055 * \frac{-27-V_{m}}{\exp \left(\frac{-27-V_{m}}{3.8}-1\right)} \\
\beta_{m}=0.94 * \exp \left(\frac{-75-V_{m}}{17}\right) & \frac{d h}{d t}=\frac{-\left(h-h_{\infty}\right)}{\tau_{h}} \\
\tau_{h}=\frac{1}{\left(\alpha_{h}+\beta_{h}\right) * \Phi} & h_{\infty}=\frac{\alpha_{h}}{\alpha_{h}+\beta_{h}} \\
\alpha_{h}=0.000457 * \exp \left(\frac{-13-V_{m}}{50}\right) & \beta_{h}=\frac{0.0065}{\exp \left(\frac{-V_{m}-15}{28}\right)+1}
\end{array}
$$

\section{Low-Threshold Ca Current (Reticular Neuron)}

Constants

$$
\begin{array}{ll}
\Phi_{m}=5^{(\text {Cels-24)/10 })} & \Phi_{h}=3^{(\text {Cels-24)/10 })} \\
E_{C a 0}=1000 * 8.31441 \frac{273.15+\text { Cels }}{2 * 96489} & {[\text { Ca }]_{\text {out }}=2} \\
\text { Cels }=36 & \text { ratio }=\frac{[\text { Ca }]_{\text {out }}}{[\text { Ca }]_{\text {in }}} \\
E_{C a}=E_{C a 0} * \log (\text { ratio }) &
\end{array}
$$

Governing Equations:

$$
\begin{array}{ll}
i_{T}=\bar{g}_{C a} * m^{2} * h *\left(V_{m}-E_{C a}\right) & \frac{d m}{d t}=\frac{-\left(m-m_{\infty}\right)}{\tau_{m}} \\
m_{\infty}=\frac{1}{1+\exp \left(\frac{-\left(V_{m}+52\right)}{7.4}\right)} & 1 \\
\tau_{m}=3+\frac{\exp \left(\frac{V_{m}+27}{10}\right)+\exp \left(\frac{-\left(V_{m}+102\right.}{15}\right) * \Phi_{m}}{\tau_{h}} & h_{\infty}=\frac{1}{1+\exp \left(\frac{V_{m}+80}{5}\right)} \\
\frac{d h}{d t}=\frac{-\left(h-h_{\infty}\right)}{\operatorname{tau}_{h}}=85+\frac{1}{\left(\operatorname { e x p } \left(\frac{V_{m}+48}{4}+\exp \left(\frac{-\left(V_{m}+407\right)}{50}\right) * \Phi_{h}\right.\right.} &
\end{array}
$$

Fast Sodium Current (Reticular and Thalamocortical Cells)

Constants:

$$
\begin{array}{ll}
V_{t r}=-50 & \mathrm{~V} 2=V_{m}-V_{t r} \\
\text { Cels }=36 & \Phi=3^{(\text {Cels-36) } / 10}
\end{array}
$$

Governing Equations: 


$$
\begin{aligned}
i_{N a} & =\bar{g}_{N a} m^{3} * h *\left(V_{m}-E_{N a}\right) & \frac{d m}{d t} & =\frac{-\left(m-m_{\infty}\right)}{\tau_{m}} \\
\tau_{m} & =\frac{1}{\left(\alpha_{m}+\beta_{m}\right) * \Phi} & m_{\text {inf }} & =\frac{\alpha_{m}}{\alpha_{m}+\beta_{m}} \\
\alpha_{m} & =0.32 * \frac{13-V 2}{\exp \left(\frac{13-V 2}{4}\right)-1} & \beta_{m} & =0.28 * \frac{v 2-40}{\exp \left(\frac{V 2-40}{5}\right)-1} \\
\frac{d h}{d t} & =\frac{-\left(h-h_{\mathrm{inf}}\right)}{\tau_{h}} & \tau_{h} & =\frac{1}{\left(\alpha_{h}+\beta_{h}\right) * \Phi} \\
h_{\mathrm{inf}} & =\frac{\alpha_{h}}{\alpha_{h}+\beta_{h}} & \alpha_{h} & =0.128 * \exp \left(\frac{17-V 2}{18}\right) \\
\beta_{h} & =\frac{4}{\exp \left(\frac{40-V 2}{5}\right)+1} & &
\end{aligned}
$$

\section{Fast Potassium Current (Reticular and Thalamocortical Cells)}

Constants:

$$
\begin{array}{ll}
V_{t r K}=-50 & \mathrm{~V} 2=V_{m}-V_{t r K} \\
\text { Cels }=36 & \Phi=3^{(\text {Cels-36) } / 10}
\end{array}
$$

Governing Equations:

$$
\begin{aligned}
i_{K} & =\bar{g}_{K} * n^{4}\left(V_{m}-E_{K}\right) & \frac{d n}{d t} & =\frac{-\left(n-n_{\infty}\right)}{\tau_{n}} \\
\tau_{n} & =\frac{1}{\left(\alpha_{n}+\beta_{n}\right) * \Phi_{n}} & n_{\infty} & =\frac{\alpha_{n}}{\alpha_{n}+\beta_{n}} \\
\alpha_{n} & =0.032 * \frac{15-V 2}{\exp \left(\frac{15-V 2}{5}\right)-1} & \beta_{n} & =0.5 * \exp \left(\frac{10-V 2}{40}\right)
\end{aligned}
$$

\section{H Current (Thalamocortical Cell)}

Constants:

$$
\begin{array}{lll}
\bar{g}_{h}=0.02 & g_{\text {inc }}=1.5 & C a_{c}=0.0015 \\
\mathrm{pc}=0.01 & \mathrm{k} 4=0.001 & E_{h}=-40 \\
p 1_{0}=\frac{1}{1+\frac{C a_{c}}{[C a]_{i n}^{n} C a}} & o 1_{0}=\frac{1}{1+\frac{\beta}{\alpha}+\frac{p 10}{p c}} & o 2_{0}=\frac{p 1_{0}}{0.001} * o 1_{0}
\end{array}
$$

Governing Equations: 


$$
\begin{array}{ll}
i_{h}=\bar{g}_{h} *\left(o 1+g_{\text {inc }} * o 2\right) *\left(V_{m}-E_{h}\right) & h_{\infty}=\frac{1}{1+\exp \left(\frac{v+75}{5.5}\right)} \\
\tau_{s}=20+\frac{1000}{\exp \left(\frac{v+71.5}{14.2}\right)+\exp \left(\frac{-(v+89)}{1.6}\right)} & \alpha=\frac{h_{\infty}}{\tau_{s}} \\
\beta=\frac{1-h_{\infty}}{t a u_{s}} & k 1=0.0004 * \frac{[C a]_{i n}{ }^{4}}{0.0015} \\
k 3 p=0.001 * \frac{p 1}{0.01} & \frac{d o 1}{d t}=\alpha *(1-o 1-o 2)-\beta * o 1 \\
\frac{d p 1}{d t}=k 1 *(1-p 1)-0.0004 * p 1 & \frac{d o 2}{d t}=k 3 p * o 1-0.0004 * o 2
\end{array}
$$

\section{Potassium A-Current (Thalamocortical Cell)}

Constants:

$$
\text { Cels }=36 \quad T_{a d j}=3^{(\text {Cels-23.5)/10 }}
$$

Governing Equations:

$$
\begin{array}{ll}
i_{A}=\bar{g}_{A} * m^{4} * h *\left(V_{m}-E_{K}\right) & \\
\tau_{m}=\frac{1.0}{\left.\exp \left(\frac{v+35.82}{19.69}\right)+\exp \left(\frac{-(v+79.69)}{12.7}\right)+0.37\right) * T_{a d j}} & m_{\infty}=\frac{1}{1+\exp \left(\frac{-(v+60)}{8.5}\right)} \\
\tau_{h}=\frac{1}{\left(\exp \left(\frac{v+46.05}{5}\right)+\exp \left(\frac{-(v+238.4)}{37.45}\right) * T_{a d j}\right.} i f\left(V_{m}<-63\right) & \tau_{h}=\frac{19.0}{T_{a d j}} i f\left(V_{m} \geq-63\right) \\
h_{\infty}=\frac{1}{1+\exp \left(\frac{v+78}{6}\right)} & \frac{d m}{d t}=\frac{-\left(m-m_{\infty}\right)}{\tau_{m}} \\
\frac{d h}{d t}=\frac{-\left(h-h_{\infty}\right)}{\tau_{h}} &
\end{array}
$$

\section{Low-Threshold Ca Current (Thalamocortical Cell)}

Constants:

$$
\begin{array}{ll}
\text { Cels }=36 & \Phi_{m}=3.55^{(\text {Cels-24) } / 10} \\
\Phi_{h}=3^{(\text {Cels-24)/10 }} & E_{C a 0}=1000 * 8.31441 * \frac{273.15+\text { Cels }}{2 * 96489} \\
\text { ratio }=\frac{C a_{0}}{[C a]_{i n}} & E_{C a}=E_{C a 0} * \log (\text { ratio })
\end{array}
$$

Governing Equations: 


$$
\begin{array}{ll}
i_{T}=\bar{g}_{C a} * m^{2} * h *\left(V_{m}-E_{C a}\right) & \frac{d m}{d t}=\frac{-\left(m-m_{\infty}\right)}{\tau_{m}} \\
m_{\infty}=\frac{1}{1+\exp \left(\frac{-(v+59)}{6.2}\right)} & \frac{d h}{d t}=\frac{-\left(h-h_{\infty}\right)}{\tau_{h}} \\
\tau_{h}=\frac{30.8+\left(211.4+\exp \left(\frac{\left(V_{m}+115.2\right)}{5}\right)\right)}{\left(1+\exp \left(\frac{V_{m}+86}{3.2}\right) * \Phi_{h}\right.} & \\
h_{\infty}=\frac{1}{1+\exp \left(\frac{V_{m}+83}{4}\right)} & 1 \\
\tau_{m}=\frac{1}{\left(\exp \left(\frac{-\left(V_{m}+131.6\right)}{16.7}\right)+\exp \left(\frac{V_{m}+16.8}{18.2}\right)+0.612\right) * \Phi_{m}}
\end{array}
$$

\section{Calcium Dynamics (All Cells)}

Constants:

$$
\begin{aligned}
& C a_{\infty}=0.00024 \quad K_{T}=0.0001 \quad K_{d}=0.0001 \\
& \text { drive }_{0}=\frac{10}{2 * 96489}
\end{aligned}
$$

Governing Equations:

$$
\begin{gathered}
\text { drive }= \begin{cases}- \text { drive }_{0} * \frac{i_{T}}{D} & \text { ifdrive }>0 \\
\text { drive }=0 & \text { ifdrive } \leq 0\end{cases} \\
\frac{d[C a]_{\text {in }}}{d t}=\text { drive }+\frac{C a_{\infty}-[C a]_{i n}}{\tau_{r}}
\end{gathered}
$$

\section{A.2.1 Pyramidal Cell}

The two cortical cells (PY and IN) are two compartment models. They have a dendritic compartment and an axosomatic compartment. These neurons will therefore be divided into three sections: axosomatic, dendritic, and coupling equations.

\section{Dendritic}

Constants: 


$$
\begin{array}{llll}
\bar{g}_{N a}=0.8 & E_{N a}=50 & \bar{g}_{K m}=0.01 & E_{K m}=-90 \\
\bar{g}_{K C a}=0.3 & E_{K C a}=-90 & \bar{g}_{H V A}=0.01 & E_{C a}=140 \\
\bar{g}_{L}=0.0333 & E_{L}=-68 & \bar{g}_{K L}=0.0025 & E_{K}=-95 \\
\bar{g}_{N A P}=3.5 & D=1 & \tau_{r}=165 &
\end{array}
$$

Governing Equations:

$i_{\text {dend }}=-\bar{g}_{L} *\left(V_{\text {dend }}-E_{L}\right)-i_{H V A}-i_{K C a}-i_{K m}-i_{N a}-i_{N a P}-\bar{g}_{K L} *\left(V_{\text {dend }}-K_{K}\right)$

\section{Axosomatic}

Constants:

$$
\begin{aligned}
& \bar{g}_{N a}=3000 \quad E_{N a}=50 \quad \bar{g}_{K v}=200 \quad E_{K v}=-90 \\
& \bar{g}_{N a P}=15
\end{aligned}
$$

Governing Equations:

$$
\begin{gathered}
g 1_{s o m a}=\bar{g}_{N a}+\bar{g}_{K v}+\bar{g}_{N a P} \\
g 2_{s o m a}=\bar{g}_{N a} * E_{N a}+\bar{g}_{K v} * E_{K v}+\bar{g}_{N a P} * E_{N a}+6.74172 \\
i_{s o m a}=-i_{N a}-i_{K v}-i_{N a P}
\end{gathered}
$$

\section{Coupling Equations}

Constants:

$$
\begin{aligned}
& \kappa=10000 \quad C=0.75 \quad \rho=165 \quad S_{\text {soma }}=0.00006 \\
& S_{\text {dend }}=S_{\text {soma }} * \rho
\end{aligned}
$$

Governing Equations:

$$
V_{\text {soma }}=\frac{V_{\text {dend }}+\kappa * S_{\text {soma }} * g 2_{\text {soma }}}{1+\kappa * S_{\text {sp }, a} * g 1_{\text {soam }}}
$$




$$
\frac{d V_{d e n d}}{d t}=\frac{1}{C} *\left(i_{\text {dend }}+\frac{V_{\text {soma }} * V d e n d}{\kappa * S_{\text {dend }}}\right)
$$

\section{A.2.2 Interneuron}

\section{Dendritic}

Constants:

$$
\begin{array}{llll}
\bar{g}_{N a}=0.8 & E_{N a}=50 & \bar{g}_{K m}=0.01 & E_{K m}=-90 \\
\bar{g}_{K C a}=0.3 & E_{K C a}=-90 & \bar{g}_{H V A}=0.01 & E_{C a}=140 \\
\bar{g}_{L}=0.0333 & E_{L}=-70 & \bar{g}_{K L}=0 & E_{K}=-95 \\
\bar{g}_{N A P}=3.5 & D=1 & \tau_{r}=165 &
\end{array}
$$

Governing Equations:

$i_{\text {dend }}=-\bar{g}_{L} *\left(V_{\text {dend }}-E_{L}\right)-i_{H V A}-i_{K C a}-i_{K m}-i_{N a}-i_{N a P}-\bar{g}_{K L} *\left(V_{\text {dend }}-K_{K}\right)$

\section{Axosomatic}

Constants:

$$
\begin{aligned}
& \bar{g}_{N a}=2500 \quad E_{N a}=50 \quad \bar{g}_{K v}=200 \quad E_{K v}=-90 \\
& \bar{g}_{N a P}=0
\end{aligned}
$$

Governing Equations:

$$
\begin{gathered}
g 1_{s o m a}=\bar{g}_{N a}+\bar{g}_{K v}+\bar{g}_{N a P} \\
g 2_{s o m a}=\bar{g}_{N a} * E_{N a}+\bar{g}_{K v} * E_{K v}+\bar{g}_{N a P} * E_{N a}+6.74172 \\
i_{s o m a}=-i_{N a}-i_{K v}-i_{N a P}
\end{gathered}
$$

\section{Coupling Equations}


Constants:

$$
\begin{aligned}
& \kappa=10000 \quad C=0.75 \quad \rho=50 \quad S_{\text {soma }}=0.00006 \\
& S_{\text {dend }}=S_{\text {soma }} * \rho
\end{aligned}
$$

Governing Equations:

$$
\begin{gathered}
V_{\text {soma }}=\frac{V_{\text {dend }}+\kappa * S_{\text {soma }} * g 2_{\text {soma }}}{1+\kappa * S_{\text {sp }, a} * g 1_{\text {soam }}} \\
\frac{d V_{\text {dend }}}{d t}=\frac{1}{C} *\left(i_{\text {dend }}+\frac{V_{\text {soma }} * V d e n d}{\kappa * S_{\text {dend }}}\right)
\end{gathered}
$$

\section{A.2.3 Reticular Neuron}

Constants:

$$
\begin{array}{llll}
\bar{g}_{N a}=100 & E_{N a}=50 & \bar{g}_{K}=10 & E_{K}=-95 \\
\bar{g}_{C a}=2.3 & E_{C a} \text { calcuated in } i_{T} & E_{L}=-77 & \bar{g}_{L}=0.05 \\
\bar{g}_{K L}=0.005 & V_{t r}=-50 & V_{t r K}=-50 & D=1
\end{array}
$$

Governing Equation:

$$
\frac{V_{m}}{d t}=-\bar{g}_{l}\left(V_{m}-E_{l}\right)-i_{T}-i_{N a}-i_{K}-\bar{g}_{K l}\left(V_{m}-E_{K}\right)
$$

\section{A.2.4 Thalmocortical Cell}

Constants: 


$$
\begin{array}{llll}
\bar{g}_{N a}=90 & E_{N a}=50 & \bar{g}_{K}=12 & E_{K}=-95 \\
\bar{g}_{C a}=2.3 & E_{C a} \text { calcuated in } i_{T} & \bar{g}_{A}=0 & D=2 \\
p c=0.007 & k 4=0.001 & g_{i n c}=2 & \bar{g}_{h}=0.0017 \\
\bar{g}_{L}=0.01 & E_{L}=-70 & \bar{g}_{K L}=0.03 & V_{t r}=-40 \\
V_{t r K}=-25 & & &
\end{array}
$$

Governing Equation:

$$
\frac{V_{m}}{d t}=-\bar{g}_{l}\left(V_{m}-E_{l}\right)-i_{T}-i_{h}-i_{N a}-i_{K}-i_{A}-\bar{g}_{K l}\left(V_{m}-E_{K}\right)
$$

\section{B Synaptic Models}

\section{B.1 Point Source Models}

\section{B.1.1 GABA Type A}

Constants:

$$
\begin{array}{lll}
E_{G A B A}=-70 & \mathrm{R}=0 & \mathrm{C}=0 \\
C_{\text {max }}=0.5 & C_{d u r}=0.3 & \text { Deadtime }=1 \\
R_{0}=0 & R_{1}=0 & \alpha=10.5 \\
\beta=0.166 & \text { lastrelease }=-100 & R_{\infty}=\frac{C_{\max } * \alpha}{C_{\max } * \alpha+\beta} \\
R_{\tau}=\frac{1}{\alpha * C_{\max }+\beta} & \text { Prethresh }=0 &
\end{array}
$$

Governing Equations:

$$
\begin{gathered}
q=(t-\text { lastrelease })-C_{d u r} \\
\text { if }(q>\text { Deadtime }) \\
\text { if }\left(V_{\text {pre }}>\text { Prethresh }\right) \\
C=C_{\max }
\end{gathered}
$$




$$
\begin{aligned}
& \quad R_{0}=R \\
& \quad \text { lastrelease }=t \\
& \text { elseif }\left(C==C_{\text {max }}\right) \\
& R_{1}=R \\
& \quad C=0 \\
& \text { if }(C>0) \\
& \quad R=R_{\text {infty }}+\left(R_{0}-R_{\text {infty }}\right) * \text { exptable }\left(-(t-\text { lastrelease }) / R_{\tau}\right) \\
& \text { else } \\
& R=R_{1} * \text { exptable }\left(-\beta *\left(t-\left(\text { lastrelease }+C_{\text {dur }}\right)\right)\right) \\
& I=g_{G A B A_{A}} * R *\left(V_{\text {post }}-E_{G A B A}\right)
\end{aligned}
$$

Where exptable is a modified exponential function defined as follows:

$$
\operatorname{exptable}(z)= \begin{cases}\exp (z) & \text { if }-10<z<10 \\ 0 & \text { if }-10>z<10\end{cases}
$$

\section{B.1.2 GABA Type B}

Constants and Initial Conditions:

$$
\begin{array}{llll}
C_{d u r}=0.3 & K_{1}=0.52 & K_{2}=0.0013 & K_{3}=0.098 \\
K_{4}=0.033 & \text { lastrelease }=-10000000 & \mathrm{C}=0 & r_{0}=0 \\
g_{0}=0 & E_{G A B A}=-95 & C_{\max }=0.5 & \text { Deadtime }=1 \\
\text { Prethresh }=0 & K_{d}=100 & \mathrm{n}=4 &
\end{array}
$$

Governing Equations:

$$
\begin{aligned}
& G_{n}=g^{n} \\
& G_{n 1}=\frac{G_{n}}{G_{n}+K_{d}} \\
& q=(t-\text { lastrelease })-C_{d u r}
\end{aligned}
$$




$$
\begin{aligned}
& \text { if }(q>\text { Deadtime }) \\
& \text { if }\left(V_{\text {pre }}>\text { Prethresh }\right) \\
& C=C_{\text {max }} \\
& \text { lastrelease }=t \\
& \text { elseif }\left(C==C_{\text {max }}\right) \\
& C=0 \\
& \frac{d r}{d t}=K_{1} * C *(1-r)-r * K_{2} \\
& \frac{d g}{d t}=K_{3} * r-K_{4} * g \\
& I=g_{G A B A_{B}} * G_{n 1} *\left(V_{\text {post }}-E_{G A B A}\right.
\end{aligned}
$$

\section{B.1.3 AMPA}

Constants and Initial Conditions:

$$
\begin{array}{llll}
\mathrm{R}=0 & \mathrm{C}=0 & R_{0}=0 & R_{1}=0 \\
\text { lastrelease }=-100 & \text { lastspike }=-100 & \mathrm{~s}=1 & E_{A M P A}=0 \\
C_{d u r}=0.3 & C_{\max }=0.5 & \text { Deadtime }=1 & C_{d e l}=0 \\
\text { Prethresh }=0 & \alpha=0.94 & \beta=0.18 & \\
R_{\infty}=\frac{C_{\max } * \alpha}{C_{\max } * \alpha+\beta} & & & \\
R_{\tau}=\frac{1}{\alpha * C_{\max }+\beta} & &
\end{array}
$$

Governing Equations:

$$
\begin{aligned}
& q=(t-\text { lastrelease })-C_{d u r} \\
& \text { if }(q>\text { Deadtime }) \\
& \text { if }\left(V_{\text {pre }}>\text { Prethresh }\right) \\
& \text { if }(t-\text { lastspike })>\left(C_{d e l}+C_{d u r}\right) \\
& \text { lastspike }=t \\
& s=1
\end{aligned}
$$




$$
\begin{gathered}
\text { if }(s==1) \wedge(t-\text { lastspike })>C_{\text {del }} \\
s=0 \\
C=C_{\text {max }} \\
R_{0}=R \\
\text { lastrelease }=t \\
\text { elseif }\left(C==C_{\text {max }}\right) \\
R_{1}=R \\
C=0 \\
\text { if }(C>0) \quad \\
R=R_{\text {infty }}+\left(R_{0}-R_{\text {infty }}\right) * \text { exptable }\left(-(t-\text { lastrelease }) / R_{\tau}\right) \\
\text { else } \\
R=R_{1} * \text { exptable }\left(-\beta *\left(t-\left(\text { lastrelease }+C_{\text {dur }}\right)\right)\right) \\
I=g_{\text {AMPA }} * R *\left(V_{\text {post }}-E_{\text {AMPA }}\right)
\end{gathered}
$$

\section{B.1.4 AMPA Type D2}

Constants and Initial Conditions:

$$
\begin{array}{llll}
\mathrm{R}=0 & \mathrm{C}=0 & R_{0}=0 & R_{1}=0 \\
\text { lastrelease }=-10000 & \text { lastrelease }_{1}=-10000 & \mathrm{E}=1 & \mathrm{~s}=1 \\
g_{1}=0.00006 & \text { newrelease }=0 & \mathrm{Use}=0.07 & T_{r}=700 \\
\tau=50 & \text { factor }=1 & E_{\text {AMPA }}=0 & C_{d u r}=0.3 \\
C_{\max }=0.5 & \text { Deadtime }=1 & C_{\text {del }}=0 & \text { Prethresh }=0 \\
\alpha=0.94 & \beta=0.18 & \text { Period }=8000 & \\
R_{\infty}=\frac{C_{\max } * \alpha}{C_{\max } * \alpha+\beta} & & & \\
R_{\tau}=\frac{1}{\alpha * C_{\max }+\beta} & & &
\end{array}
$$

Governing Equations: 


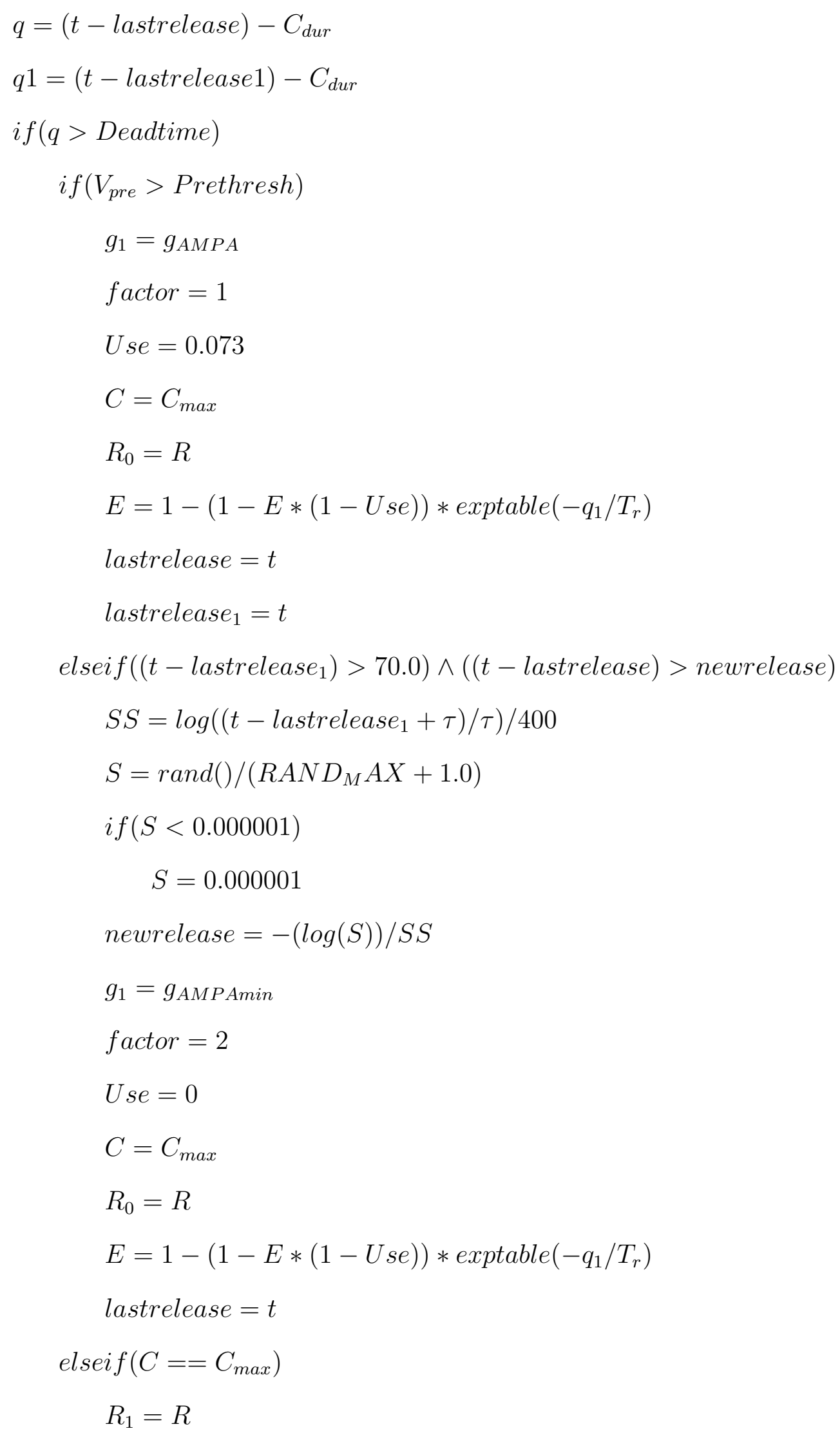




$$
\begin{aligned}
& \quad C=0 \\
& \text { if }(C>0) \\
& \quad R=R_{\text {infty }}+\left(R_{0}-R_{\text {infty }}\right) * \text { exptable }\left(-(t-\text { lastrelease }) / R_{\tau}\right) \\
& \text { else } \\
& \quad R=R_{1} * \text { exptable }\left(-\beta *\left(x-\left(\text { lastrelease }+C_{\text {dur }}\right)\right)\right) \\
& I=g_{1} * R * E *\left(V_{\text {post }}-E_{\text {AMPA }}\right.
\end{aligned}
$$

\section{B.1.5 NMDA Type D1}

Constants and Initial Conditions:

$$
\begin{array}{llll}
\mathrm{R}=0 & \mathrm{C}=0 & R_{0}=0 & R_{1}=0 \\
\text { lastrelease }=-100 & \text { lastspike }=-100 & \mathrm{~s}=1 & \mathrm{E}=1 \\
\mathrm{Use}=0.0 & T_{r}=750 & E_{N M D A}=0 & C_{d u r}=0.3 \\
C_{\max }=0.5 & \text { Deadtime }=1 & C_{\text {del }}=0 & \text { Prethresh }=0 \\
\alpha=1 & \beta=0.0067 & & \\
R_{\infty}=\frac{C_{\max } * \alpha}{C_{\max } * \alpha+\beta} & & \\
R_{\tau}=\frac{1}{\alpha * C_{\max }+\beta} & &
\end{array}
$$

Governing Equations:

$$
\begin{aligned}
& q=(t-\text { lastrelease })-C_{d u r} \\
& \text { if }(q>\text { Deadtime }) \\
& \text { if }\left(V_{\text {pre }}>\text { Prethresh }\right) \\
& \text { if }(t-\text { lastspike })>\left(C_{\text {del }}+C_{\text {dur }}\right) \\
& \text { lastspike }=t \\
& s=1 \\
& \text { if }\left((s==1) \wedge\left((t-\text { lastspike })>C_{\text {del }}\right)\right) \\
& s=0
\end{aligned}
$$




$$
\begin{gathered}
C=C_{\max } \\
R_{0}=R \\
E=1-(1-E *(1-U \text { se })) * \operatorname{exptable}\left(-q / T_{r}\right) \\
\text { lastrelease }=t \\
\text { elseif }(C==\text { Cmax }) \\
R_{1}=R \\
C=0 \\
\text { if }(C>0) \\
R=R_{\text {infty }}+\left(R_{0}-R_{\text {infty }}\right) * \text { exptable }\left(-(t-\text { lastrelease }) / R_{\tau}\right) \\
\text { else } \quad R_{1} * \text { exptable }\left(-\beta *\left(x-\left(\text { lastrelease }+C_{\text {dur }}\right)\right)\right) \\
\text { fn }=\frac{1}{\left.1+\exp \left(-\left(V_{\text {post }}+25\right)\right) / 12.5\right)} \\
I=g_{N M D A} * R * \text { fn } * E *\left(V_{\text {post }}-E_{N M D A}\right)
\end{gathered}
$$

\section{B.1.6 GABA Type A D2}

Constants and Initial Conditions:

$$
\begin{array}{llll}
E_{G A B A}=-70 & \mathrm{R}=0 & \mathrm{C}=0 & R_{0}=0 \\
R_{1}=0 & \text { lastrelease }=-10000 & \text { lastrelease }_{1}=-10000 & \mathrm{E}=1 \\
\text { newrelease }=0 & \text { Use }=0 & T_{r}=700 & \text { Period }=8000 \\
\tau=50 & \text { factor }=1 & C_{d u r}=0.3 & C_{\max }=0.5 \\
\text { Deadtime }=1 & \text { Prethresh }=0 & \alpha=10 & \beta=0.25 \\
R_{\infty}=\frac{C_{\max } * \alpha}{C_{\max } * \alpha+\beta} & & \\
R_{\tau}=\frac{1}{\alpha * C_{\max }+\beta} & &
\end{array}
$$

Governing Equations:

$$
q=(t-\text { lastrelease })-C_{d u r}
$$




$$
\begin{aligned}
& q 1=(t-\text { lastrelease } 1)-C_{d u r} \\
& \text { if }(q>\text { Deadtime }) \\
& \text { if }\left(V_{\text {pre }}>\text { Prethresh }\right) \\
& \text { factor }=1 \\
& U s e=0.07 \\
& C=C_{\max } \\
& R_{0}=R \\
& E=1-(1-E *(1-U s e)) * \operatorname{exptable}\left(-q_{1} / T_{r}\right) \\
& \text { lastrelease }=t \\
& \text { lastrelease }_{1}=t \\
& \text { elseif }((t-\text { lastrelease } 1)>70.0) \wedge(t \text { - lastrelease })>\text { newrelease }) \\
& S S=\log \left(\left(t-\text { lastrelease }_{1}+\tau\right) / \tau\right) / 400 \\
& S=\operatorname{rand}() /\left(R A N D_{M} A X+1.0\right) \\
& \text { if }(S<0.000001) \\
& S=0.000001 \\
& \text { newrelease }=-(\log (S)) / S S \\
& \text { factor }=10 \\
& U s e=0 \\
& C=C_{\max } \\
& R_{0}=R \\
& E=1-(1-E *(1-U s e)) * \operatorname{exptable}\left(-q_{1} / T_{r}\right) \\
& \text { lastrelease }=t \\
& \text { elseif }(C==C \max ) \\
& R_{1}=R \\
& C=0 \\
& \text { if }(C>0) \\
& R=R_{\text {infty }}+\left(R_{0}-R_{\text {infty }}\right) * \text { exptable }\left(-(t-\text { lastrelease }) / R_{\tau}\right)
\end{aligned}
$$


else

$$
\begin{array}{r}
R=R_{1} * \text { exptable }\left(-\beta *\left(t-\left(\text { lastrelease }+C_{d u r}\right)\right)\right) \\
I=\left(g_{G A B A_{A}} / \text { factor }\right) * E * R *\left(V_{\text {post }}-E_{G A B A}\right)
\end{array}
$$

\section{B.1.7 Externally stimulated AMPA}

Constants and Initial Conditions:

$$
\begin{array}{llll}
\alpha=0.94 & \beta=0.18 & \mathrm{R}=0 & \mathrm{C}=0 \\
R_{0}=0 & R_{1}=0 & \text { lastrelease }=-100 & \mathrm{TR}=1000 \\
\mathrm{w}=0.01 & \text { wom }=0 & C_{d u r}=0.3 & C_{\max }=0.5
\end{array}
$$

Deadtime $=1 \quad$ Prethresh $=0$

$$
\begin{aligned}
& R_{\infty}=\frac{C_{\max } * \alpha}{C_{\max } * \alpha+\beta} \\
& R_{\tau}=\frac{1}{\alpha * C_{\max }+\beta}
\end{aligned}
$$

Governing Equations:

$$
\begin{aligned}
& q=(t-\text { lastrelease })-C_{d u r} \\
& \text { if }(q>\text { Deadtime }) \\
& \text { if }((t-\text { lastrelease })>T R) \quad C=C_{\max } \\
& \quad R_{0}=R \\
& \quad \text { lastrelease }=t \\
& \text { elseif }\left(C==C_{\text {max }}\right) \\
& \quad R 1=R \\
& \quad C=0 \\
& \text { if }(C>0) \\
& R=R_{\text {infty }}+\left(R_{0}-R_{\text {infty }}\right) * \text { exptable }\left(-(t-\text { lastrelease }) / R_{\tau}\right) \\
& \text { else } \\
& R=R_{1} * \text { exptable }\left(-\beta *\left(t-\left(\text { lastrelease }+C_{\text {dur }}\right)\right)\right)
\end{aligned}
$$




$$
g=g_{A M P A_{\text {extern }}} * R
$$




\section{Bibliography}

Moshe Abeles. Local Cortical Circuits: An electrophysiological Study. Springer-Verlag, 1982.

Moshe Abeles. Corticonics: Neural Circuits of the Cerebral Cortex. Cambridge University Press, 1991.

Costas A. Anastassiou, Rodrigo Perin, Gyrgy Buzski, Henry Markram, and Christof Koch. Cell type- and activity-dependent extracellular correlates of intracellular spiking. Journal of Neurophysiology, 114(1):608-623, 2015. doi: 10.1152/jn.00628.2014. URL https://doi.org/10.1152/jn.00628.2014.

M Bazenhov, I Timofeev, M Steriade, and T Sejnowski. Model of thalamocortical slow-wave sleep oscillations and transitions to active states. The Journal of Neuroscience, 22:8691-8704, October 2002.

G Buzsàki, RG Bickford, G Ponomareff, LJ Thal, R Mandel, and FH Gage. Nucleus basalis and thalamic control of neocortical activity in the freely moving rat. 8(11):4007-4026, 1988. ISSN 0270-6474. doi:

10.1523/JNEUROSCI.08-11-04007.1988. URL http://www.jneurosci.org/content/8/11/4007.

György Buzsàki, Costas A. Anastassiou, and Christof Koch. The origin of extracellular fields and currents eeg, ecog, lfp and spikes. Nature Reviews Neuroscience, 13:407 - 420, 2012. doi: 10.1038/nrn3241. URL http://dx.doi.org/10.1038/nrn3241.

Nicholas T Carnevale and Michael L Hines. The NEURON Book. Cambridge University Press, 2006.

Michael X Cohen. Where does eeg come from and what does it mean? Trends in Neurosciences, 40(4):208 - 218, 2017. ISSN 0166-2236. doi: https://doi.org/10.1016/j.tins.2017.02.004. URL http: //www.sciencedirect.com/science/article/pii/S0166223617300243.

D Contreas and M Steriade. Cellular basis of eeg slow rhythms: A study of dynamic corticothalamic relationships. The Journal of Neuroscience, 15(1): 604-622, 1995.

Peter Dayan and L.F. Abbot, editors. Theoretical Neuroscience: Computational and Mathematical Medeling of Neural Systems. Cambridge, Mass ; MIT Press, 2001.

Erik De Schutter. Computational Modeling Methods for Neuroscientists. Cambridge, Mass ; Kibdib : MIT Press, 2010. 
A Delorme and S Makeig. Eeglab: an open source toolbox for analysis of single-trial eeg dynamics. Journal of Neuroscience Methods, 134:9-21, 2004.

Alain Destexhe and Denis Par. Impact of network activity on the integrative properties of neocortical pyramidal neurons in vivo. Journal of Neurophysiology, 81(4):1531-1547, 1999. doi: 10.1152/jn.1999.81.4.1531. URL https://doi.org/10.1152/jn.1999.81.4.1531.

CHris Eliasmith and Charles H. Anderson, editors. Neural Engineering: Computaion, Representation, and Dynamics in Neurobiological Systems. Cambridge, Mass; Brandfore Book: MIT Press, 2003.

Wulfram Gerstner, Werner Kistler, Richard Naud, and Liam Paninski. Neuronal Dynamics online book: From single neurons to networks and models of cognition, chapter 3.1. Cambridge University Press, 2014. URL http://neuronaldynamics.epfl.ch/online/Ch3.S1.html.

Ary L. Goldberger, Luis A. N. Amaral, Leon Glass, Jeffrey M. Hausdorff, Plamen Ch. Ivanov, Roger G. Mark, Joseph E. Mietus, George B. Moody, Chung-Kang Peng, and H. Eugene Stanley. Physiobank, physiotoolkit, and physionet. Circulation, 101(23):e215-e220, 2000. ISSN 0009-7322. doi: 10.1161/01.CIR.101.23.e215. URL http://circ.ahajournals.org/content/101/23/e215.

DPhil Gordon Shepherd, MD and MD Sten Grillner. Handbook of Brain Microcircuits. Oxford, UK, 2010. ISBN 9780195389883. URL http://oxfordmedicine.com/view/10.1093/med/9780195389883.001. $0001 /$ med-9780195389883

A Gramfort, T Papadopoulo, E Olivi, and M Clerc. Openmeeg: opensource software for quasistatic bioelectromagnetics. BioMedical Engineering OnLine, 45:9, 2010.

Arthur C Guyton and John E Hall. Textbook of Medical Physiology. W.B. Saunders Company, 9th edition edition, 1996.

LF Haas. Hans berger (1873-1941), richard catan (1842-1926), and electroencephalography. Journal of Neurology, Neurosurgery, and Psychiatry, $74(1): 9,2003$.

Espen Hagen, David Dahmen, Maria L. Stavrinou, Henrik Lindn, Tom Tetzlaff, Sacha J. van Albada, Sonja Grn, Markus Diesmann, and Gaute T. Einevoll. Hybrid scheme for modeling local field potentials from point-neuron networks. Cerebral Cortex, 26(12):4461-4496, 2016. doi: 10.1093/cercor/bhw237. URL http://dx.doi.org/10.1093/cercor/bhw237.

Hans Hallez, Bart Vanrumste, Roberta Grech, Joseph Muscat, Wim De Clercq, Anneleen Vergult, Yves D'Asseler, Kenneth P. Camilleri, Simon G. Fabri, 
Sabine Van Huffel, and Ignace Lemahieu. Review on solving the forward problem in eeg source analysis. Journal of NeuroEngineering and Rehabilitation, 4(1):46, 2007. doi: 10.1186/1743-0003-4-46. URL http://dx.doi.org/10.1186/1743-0003-4-46.

Oscar Herreras. Local field potentials: Myths and misunderstandings. Frontiers in Nueral Circuits, 10(101), 2016. doi: 10.3389/fncir.2016.00101.

David Herzfeld. Modeling and computational framework for the specification and simulation of large-scale spiking neural networks. Master's thesis, Marquette University, 2011.

M. L. Hines and N. T Carnevale. The neuron simulation environment. Neural Computation, 9(6):1179-1202, Aug 1997.

A. L. Hodgkin and W. A. H. Rushton. The electrical constants of a crustacean nerve fibre. Proceeedings of the Royal Society B, 133(873):444-479, 1946.

Alan L Hodgkin and Andrew F Huxley. A quantitative description of membrane current and its application to conduction and excitation in nerve. The Journal of Physiology, 117(4):500-544, 1952.

Eugene M. Izhikevich. Simple model of spiking neurons. IEEE Transacions on Neural Networks, 14(6):1569-1572, 2003.

Dieter Jaeger. Realistic single cell modeling - from experiment to simulation. Brains, Minds and Media, 1(2), 2005.

Paul Johns. Chapter 5 - neurons and glial cells. In Paul Johns, editor, Clinical Neuroscience, pages $61-69$. Churchill Livingstone, 2014. ISBN 978-0-443-10321-6. doi: https://doi.org/10.1016/B978-0-443-10321-6.00005-9. URL http: //wWW.sciencedirect.com/science/article/pii/B9780443103216000059.

Daniel Johnston and Samuel Miao-sin Wu. Foundations of Cellular Neurophysiology. The MIT Press, 1999.

B Kemp, AH Zwinderman, B Tuk, HAC Kamphuisen, and JJL Obery. Analysis of a sleep-dependent neuronal feedback loop: the slow-wave microcontinuity of the eeg. IEEE-BME, 47(9):1185-1194, 2000. URL https://www.physionet.org/physiobank/database/sleep-edfx/Papers/ 2000-Kemp---neurofeedback.pdf.

J Kybic, M Clerc, T Abboud, O Faugeras, R Keriven, and T Papadopoulo. A common formalism for the integral formulations of the forward eeg problem. IEEE Transactions on Medical Imaging, 24:12-28, 2005. 
R Llinas and C Nicholson. Handbook of Electroencephalography and Clinical Neurophysiology Volume 2 Part B, volume 2, chapter Analysis of Field Potentials in the Central Nervous System. Elsevier Scientific Publishing Company, 1974.

Fernando Lopes da Silva. EEG - fMRI: Physiological Basis, Technique, and Applications, chapter 2, EEG: Origin and Measurement, pages 19-38. Springer-Verlag Berlin Heidelberg, 2010.

Rafeal Lorente de No. A study of nerve physiology. Rockefeller Institute, 132, 1947.

Zachary F. Mainen and Terrence J. Sejnowski. Influence of dendritic structure on firing pattern in model neocortical neurons. Nature, 382:363-366, July 1996.

Peter Marsalek, Christof Koch, and John Maunsell. On the relationship between synaptic input and spike output jitter in individual neurons. Proceeding of the National Acadamy of Science USA, 94:735-740, January 1997.

Robert A. McDougal, Thomas M. Morse, Ted Carnevale, Luis Marenco, Rixin Wang, Michele Migliore, Perry L. Miller, Gordon M. Shepherd, and Michael L. Hines. Twenty years of modeldb and beyond: building essential modeling tools for the future of neuroscience. Journal of Computational Neuroscience, 42(1): 1-10, Feb 2017. ISSN 1573-6873. doi: 10.1007/s10827-016-0623-7. URL https://doi.org/10.1007/s10827-016-0623-7.

Karl E. Misulis and Thomas C. Head. Essentials of Clinical Neurophysiology. Butterworth Heinemann, third edition edition, 2003.

MS Mourtazaev, AH Kemp, B andZwinderman, and HAC Kamphuisen. Age and gender affect different characteristics of slow waves in the sleep eeg. Sleep, 18 (7):557-564, 1995 .

Shingo Murakami and Yoshio Okada. Contributions of principal neocortical neurons to magnetoencephalography and electroencephalography signals. The Journal of Physiology, 575(3):925-936, 2006.

Shingo Murakami, Tongsheng Zhang, Akira Hirose, and Yoshio Okada. Physiological origins of evoked magnetic fields and extracellular field potentials produced by guinea-pig ca3 hippocampal slices. The Journal of Physiology, 544(1):237-251, 2002.

T. Musha and M. Yamamoto. 1/f fluctuations in biological systems. In Engineering in Medicine and Biology Society, 1997. Proceedings of the 19th Annual International Conference of the IEEE, volume 6, pages 2692-2697 vol.6, Oct 1997.

Paul L Nunez and Ramesh Srinivasan. Electric Fields of the Brain. Oxford University Press, second edition edition, 2006. 
Piotr Olejniczak. Neurophysiologic basis of eeg. Journal of Clinical Neurophysiology, 23(3):186-189, June 2006.

Wilfrid Rall. Handbook of Physiology; Section 1: The Nervous System; Volume 1: Cellular Biology of Neurons, Part1; Chapter 3, chapter Core Conductor Theory and Cable Properties of Neurons, pages 39-98. Waverly Press Inc, 1959.

Michael W. Reimann, Costas A. Anastassiou, Rodrigo Perin, Sean L. Hill, Henry Markram, and Christof Koch. A biophysically detailed model of neocortical local field potentials predicts the critical role of active membrane currents. Neuron, 79(2):375 - 390, 2013. ISSN 0896-6273. doi: https://doi.org/10.1016/j.neuron.2013.05.023. URL http: //www.sciencedirect.com/science/article/pii/S0896627313004431.

JJ Riera, T Ogawa, T Goto, A Sumiyoshi, H Nonaka, A Evans, H Miyakawa, and R Kawashima. Pitfalls in the dipolar model for the neocortical eeg sources. Journal of Neurophysiology, 180(4):956-975, 2012.

Nelson Spruston. Pyramidal neurons: dendritic structure and synaptic integration. Nature Reviews Neuroscience, 9:206-221, 2008. URL http://dx.doi.org/10.1038/nrn2286.

M Steriade, A Nunez, and F Amzica. A novel slow (; $1 \mathrm{hz}$ ) oscillation of neocortical neurons in vivo: depolarizing and hyperpolarizing components. Journal of Neuroscience, 13(8):3252-3265, 1993. ISSN 0270-6474. doi: 10.1523/JNEUROSCI.13-08-03252.1993. URL http://www.jneurosci.org/content/13/8/3252.

Greg Stuart, Nelson Spruston, and Michael Husser. Dendrites. Oxford ; New York : Oxford University Press, 2008., 2008. ISBN 9780198566564.

Mototaka Suzuki and Matthew Evan Larkum. Dendritic calcium spikes are clearly detectable at the cortical surface. In Nature Communications, 2017.

F Tadel, S Baillet, JC Mosher, D Pantazis, and RM Leahy. Brainstorm: A user-friendly application for meg/eeg analysis. Computational Intelligence and Neuroscience, 2011:13, 2011.

John T. Weber, Chris I. De Zeeuw, David J. Linden, and Christian Hansel. Long-term depression of climbing fiber-evoked calcium transients in purkinje cell dendrites. Proceedings of the National Academy of Sciences, 100(5): 2878-2883, 2003. ISSN 0027-8424. doi: 10.1073/pnas.0536420100. URL http://www . pnas . org/content/100/5/2878.

Thomson William. On the theory of the electric telegraph. Proceedings of the Royal Society of London, 7:382-399, 1854. 
Junhong Zhou, Dongdong Liu, Xin Li, Jing Ma, Jue Zhang, and Jing Fang. Pink noise: Effect on complexity synchronization of brain activity and sleep consolidation. Journal of Theoretical Biology, 306:68 - 72, 2012. ISSN 0022-5193. doi: https://doi.org/10.1016/j.jtbi.2012.04.006. URL http: //wWw.sciencedirect.com/science/article/pii/S0022519312001798. 